\title{
Dự án Phát triển bộ cơ sở dữ liệu khoa học ngành toán của Việt Nam: Một số kết quả sơ bộ ban đâu từ CSDL SciMath
}

\author{
Preliminary findings from SciMath-a database of Vietnam \\ mathematics
}

Tài liệu kỹ thuật phục vụ cuộc họp Hội đồng khoa học của VIASM ngày

13/11/2020 (No. VIASM-AISDL-20.01)

Tại VIASM, Ngõ 157 Chùa Láng, Láng Thượng, Đống Đa, Hà Nội

Chủ trì cuộc họp

GS. Ngô Bảo Châu

PGS. Lê Minh Hà

\section{Technical Report No. VIASM-AISDL-20.01}

November 13, 2020

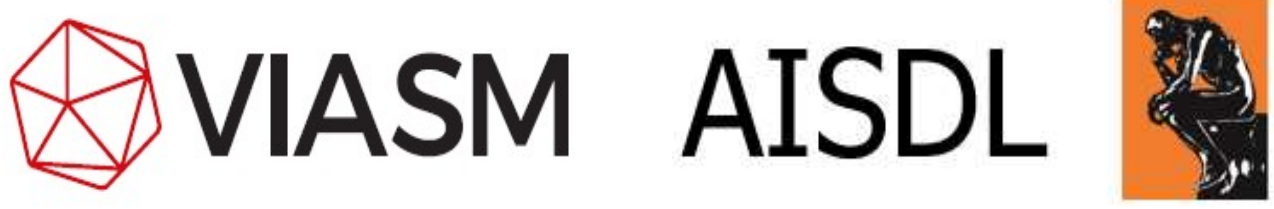

Vietnam Institute for Advanced Study in Mathematics

AI for Social Data Lab 


\section{Tổng quát CSDL SciMath}

Dự án "Phát triển bộ cơ sở dữ liệu khoa học ngành toán của Việt Nam" bắt đầu được triển khai xây dựng hệ thống vào năm 2019. Cơ sở dữ liệu (CSDL) SciMath đã được nhóm dự án xây dựng từ ngày tháng 8 năm 2019. Ngày 16-12-2019, website nhập liệu SciMath (Địa chỉ truy cập: http://scimath.aisdl.com) bắt đầu được kiểm tra chạy thử, và những điểm dữ liệu đầu tiên chính thức được lưu trong hệ thống vào ngày 23-12-2019. Đến đầu năm 2020, dự án đã chính thức được khởi động. Mặc dù trải qua thời gian khó khăn do COVID-19, đội dữ liệu vẫn tiếp tục công việc trong thời gian cách ly xã hội để có thể đảm bảo tiến độ dự án. Đến tháng 9 và tháng 10 năm 2020, nhóm dữ liệu và các đại diện VIASM đã có các buổi làm việc chi tiết về tiến độ và góp ý cho dự án. Hình ảnh các buổi làm việc có thể xem ở phần Phụ lục 1 .

CSDL thu thập công bố của các nhà toán học Việt Nam thỏa mãn hai điều kiện sau:

1. Có ít nhất một tác giả là người Việt Nam, và từng học tập ở Việt Nam.

2. Là các công trình được chỉ mục hóa bởi MathSciNet, zbMATH, hoặc được công bố trên các tạp chí được chỉ mục hóa bởi Scopus hoặc ISI.

\subsection{Cấu trúc CSDL SciMath}

Hệ thống SciMath bao gồm 3 thành phần chính (biên tập dữ liệu - curation, phân tích dữ liệu - analyze, và trình bày - presentation) được xây dựng, cài đặt theo các mức phân quyền khác nhau.

- Hệ thống biên tập dữ liệu bao gồm các công cụ tự động thu thập dữ liệu nghiên cứu khoa học từ các nguồn trực tuyến (scientific literature crawler), các công cụ nhập liệu dữ liệu, biên tập, lọc và kiểm tra dữ liệu.

- Hệ thống phân tích dữ liệu bao gồm các công cụ truy vấn dữ liệu, trích xuất, xây dựng báo cáo thống kê và các báo cáo theo chủ đề

- Hệ thống trình bày dữ liệu bao gồm cổng thông tin hiển thị và tìm kiếm cho cộng đồng và các API kết nối dữ liệu cho các tổ chức khác

Hiện nay, hệ thống biên tập dữ liệu đang hoạt động để đảm bảo tiến độ nhập dữ liệu. Hệ thống phân tích và trình bày dữ liệu đang tiếp tục được xây dựng và kiểm tra. 
Đây là cơ sở dữ liệu trung tâm lưu trữ toàn bộ các dữ liệu về tác giả/nhà khoa học, các tổ chức nghiên cứu, các bài báo/bài nghiên cứu, các tạp chí/nhà xuất bản... theo tiêu chí dữ liệu của hệ thống.

Các dữ liệu này được nhập từ nhiều nguồn khác nhau như dữ liệu từ các cơ sở dữ liệu nghiên cứu (Google Scholar, ZbMath, hay Mathscinet), dữ liệu đóng góp của các nhà khoa học và tổng hợp từ các nguồn dữ liệu trực tuyến được thu thập tự động sau đó biên tập, làm sạch và nhập vào hệ thống. Cấu trúc CSDL như sau:

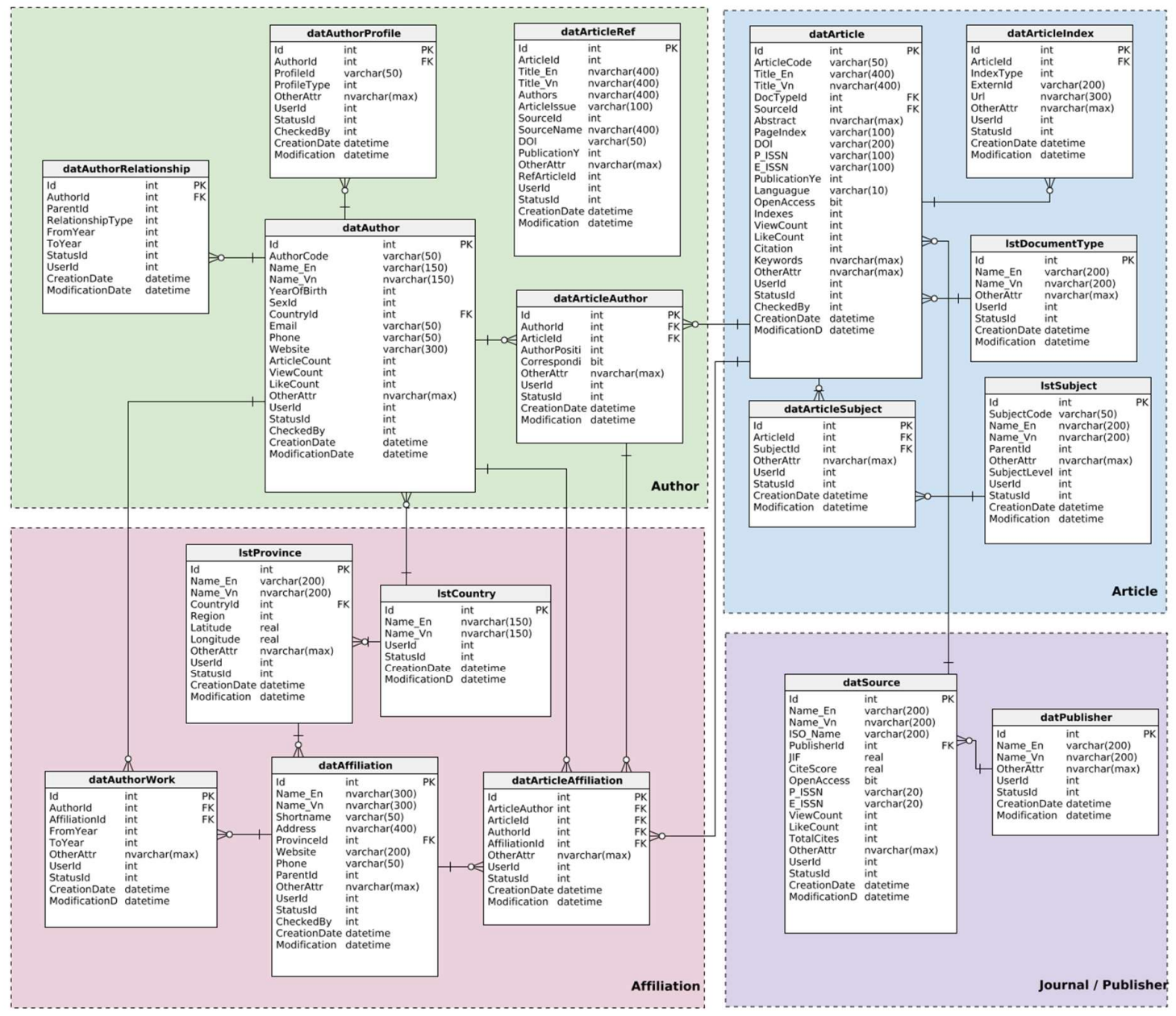

Hình 1. Cấu trúc CSDL SciMath

Cơ sở dữ liệu SciMath chia làm 4 nhóm dữ liệu cơ bản.

- Article: dữ liệu bài / nghiên cứu khoa học

Dữ liệu Article sẽ bao gồm các miền sau: tên bài, loại ẩn phẩm (bài báo, sách hoặc bài hội thảo), năm xuất bản, tên tạp chí, DOI/link, Abtract, từ khóa, chuyên 
ngành, tác giả và cơ quan. Trong đó, tên bài, năm xuất bản, tên tạp chí là các miền bắt buộc điền.

- Author: dữ liệu tác giả / nhà nghiên cứu

Dữ liệu tác giả bao gồm các miền sau: Tên tác giả, giới tính, quốc tịch, cơ quan và một số thông tin khác như email, số điện thoại. Mỗi tác giả được ghi nhận tại cơ sở dữ liệu sẽ được gắn một mã nhận diện (ID) dựa trên giới tính, quốc tịch và thứ tự nhập. Ví dụ, tác giả $\mathrm{A}$ là người Việt Nam, giới tính nữ do vậy sẽ có mã vf.999 (v: Việt Nam; f: female - nữ). Nếu A là người nước ngoài, giới tính nam sẽ được mã fm.999 (f: foreign - người nước ngoài; m: male - nam). Con số 999 thể hiện đây là tác giả thứ 999 được nhập vào cơ sở dữ liệu.

Các thông tin khác được người thu thập sử dụng làm cơ sở để phân biệt các tác giả trong trường hợp trùng tên hoặc tên viết tắt.

- Affiliation: dữ liệu tổ chức / cơ quan.

- Source / Publisher: dữ liệu tạp chí, nhà xuất bản

Trong đó, Article được coi là điểm trung tâm, và xoay quanh đó các nhóm dữ liệu khác sẽ xây dựng và thu thập. Bốn thông tin này là bắt buộc để một bài báo được ghi nhận vào cơ sở dữ liệu. Chúng cũng liên kết với nhau thông qua điểm trung tâm là Article.

\subsection{Quy trình nhập liệu}

\subsubsection{Các giai đoạn nhập liệu}

Quá trình nhập liệu bao gồm hai giai đoạn, cụ thể như sau:

Giai đoạn 1: Nhập dũu liệu tù Reference Data:

Các điểm dữ liệu đầu tiên được dựa trên hơn 4500 dòng dữ liệu đã được thu thập bằng tự động từ trước trong phần Reference Data. Mỗi bài viết sẽ được kiểm tra xác nhận thông tin bằng Google Scholar, zbMath, Mathscinet và các nguồn trên mạng khác, sau đó mới tiến hành nhập vào CSDL SciMath theo từng nhóm dữ liệu.

4500 dòng dữ liệu này chỉ có những công bố được xuất bản trước và trong năm 2008.

Giai đoạn 2: Tìm kiếm thủ công:

Ở giai đoạn này, người nhập tìm kiếm dữ liệu công bố của các tác giả Việt Nam thông qua, nhưng không giới hạn, các kênh sau: Google Scholar, website của các trường 
đại học và các viện nghiên cứu về Toán (trang giới thiệu về công bố nghiên cứu và nhân sự), zbmath, website cá nhân của tác giả (ví dụ: Google site, Blog). Các trang trên sẽ được ghi lại trên file excel như một kho trữ dữ liệu cần kiểm tra.

Ngoài ra, người nhập cũng sử dụng thông tin từ những tác giả đã được ghi nhận từ giai đoạn trước. Từ đó, tìm kiếm, bổ sung những công bố chưa được nhập.

Ở giai đoạn này, nhân viện tập trung khai thác những thông tin dễ tìm kiếm nên cơ sở dữ liệu vẫn còn chưa đầy đủ.

\subsubsection{Quy trình nhập liệu:}

Về cơ bản, quy trình nhập dữ liệu được thể hiện ở biểu đồ dưới đây:

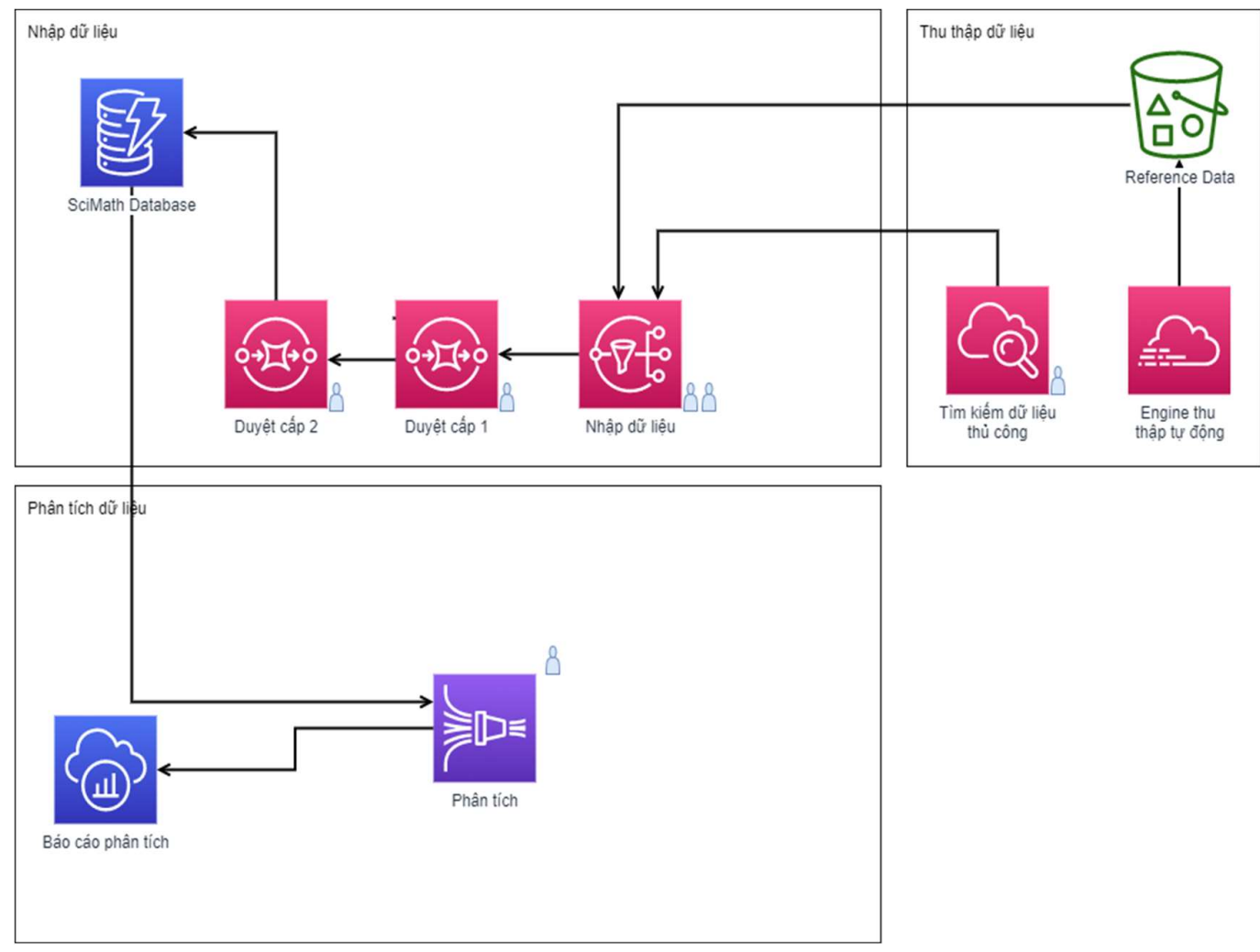

Hình 2. Quy trình nhập liệu SciMath

Thu thập dữ liệu:

- Dữ liệu được thu thập tự động từ zbMath, Mathscinet và Google Scholar lấy các ấn phẩm ngành toán của tác giả Việt Nam đưa vào cơ sở dữ liệu tham khảo.

- Dữ liệu được tìm kiếm từ các ấn phẩm đã xuất bản, trang của các tổ chức, trường, viện nghiên cứu. 
Nhập dữ liệu:

- Nhân viên nhập liệu tiến hành kiểm tra và nhập dữ liệu đã thu thập.

- Bổ sung các dữ liệu tác giả, quan hệ cũng như các dữ liệu thuộc tính từ các nguồn khác.

- Hệ thống tự động kiểm tra tính hợp lệ, dữ liệu trùng lặp tác giả, ấn phẩm...

Duyệt dữ liệu:

- Duyệt dữ liệu 2 cấp, cấp 1 duyệt từ dữ liệu nhập, cấp 2 có thể duyệt từ dữ liệu cấp 1 hoặc từ dữ liệu nhập.

- Thao tác duyệt kiểm tra tính hợp lệ của dữ liệu.

- Kiểm tra các ấn phẩm, tác giả trùng lặp.

- Dữ liệu sau khi duyệt được đưa vào cơ sở dữ liệu SciMath.

Dù đã có bước tự động kiểm tra của hệ thống, việc duyệt dữ liệu đóng vai trò rất quan trọng để có CSDL chất lượng. Thực hiện kiểm tra 2 lần giúp giảm đi các sai sót khi nhập thủ công như trùng lặp bài, tác giả, ấn phẩm. Quá trình duyệt dữ liệu thủ công này sẽ kiểm tra từng đơn vị dữ liệu liên quan đến bài báo, và tiến hành chỉnh sửa ngay khi xuất hiện các thông tin sai, trùng lặp, hoặc loại bỏ nếu bài không phù hợp với tiêu chí của Scimath. Ví dụ, để tránh các công bố bị lặp do có kí tự toán học, người kiểm tra sẽ tìm kiếm bài báo sử dụng các cụm từ/ chữ cái khác kí hiệu toán học trong tiêu đề bài và tra danh sách bài được hiện trên CSDL. Cách tra cứu và duyệt với các nhóm dữ liệu khác (tạp chí, tác giả, ...) cũng diễn ra tương tự để đảm bảo chất lượng CSDL cao nhất có thể.

Phân tích dữ liệu:

- Các bảng dữ liệu tra cứu, đồ thị được lấy trực tiếp từ cơ sở dữ liệu SciMath phục vụ công tác nghiên cứu.

\section{Kết quả sơ bộ}

Cơ sở dữ liệu được bắt đầu nhập data từ ngày 23/12/2019 và chốt dữ liệu đợt 1 vào ngày 5/11/2020. Trong quãng thời gian này, cơ sở dữ liệu đã ghi nhận 8372 công bố, 3058 tác giả (trong đó 1566 tác giả Việt Nam và 1492 tác giả nước ngoài), 1267 tạp chí/ sách/ kỉ yếu. Lưu ý, đây mới là kết quả được tổng kết tính đến ngày $5 / 11 / 2020$, vì vậy, trong 
nhiều thống kê có thể thấy dữ liệu một số giai đoạn bị hụt (dù không nhiều) do cơ sở dữ liệu còn chưa đầy đủ.

\subsection{Số bài báo}

Nhìn chung, số lượng các bài báo khoa học trước năm thập niên 60s tương đối thưa thớt. Từ năm 1947 đến năm 1960, chỉ có 9 công trình nghiên cứu được thực hiện bởi 3 tác giả. Trong đó 5 bài báo khoa học đầu tiên được viết bởi tác giả Lê Văn Thiêm trước và trong năm 1950. Bài báo khoa học đầu tiên được công bố vào năm 1947 liên quan đến hàm biến phức (Functions of a complex variable) (Le, 1947). Bài báo bằng tiếng Đức được đăng trên tạp chí Commentarii Mathematici Helvetici của Hiệp Hội Toán Học Thụy Sĩ (Swiss Mathematical Society). Bài báo này cũng như các công trình Toán học trước những năm 2000 được công bố bởi nhiều thứ tiếng khác nhau như Đức, Pháp hay tiếng Nga.

\section{Publications Over Time}

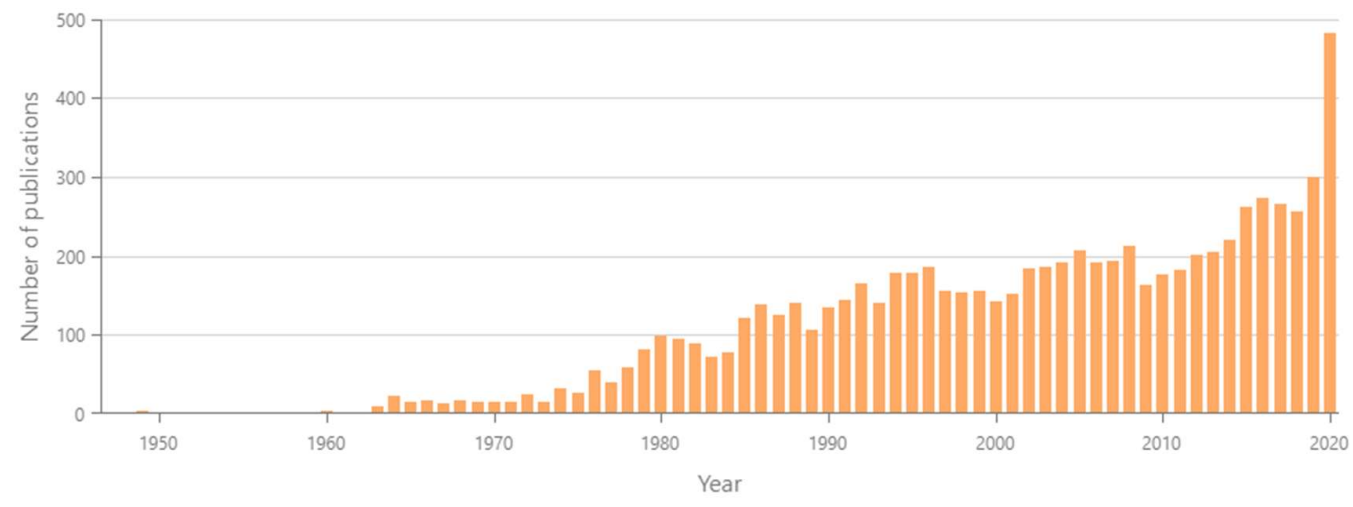

Hình 3. Số lượng ấn phẩm theo năm (Tính đến 5/11/2020)

Các bài báo khoa học còn lại được xuất bản vào cuối thập niên $50 \mathrm{~s}$ và đầu thập niên 60s bởi 2 tác giả Đặng Đình Áng và Nguyễn Đình Ngọc. Kể từ năm 1970, ngành Toán Học ghi nhận tốc độ phát triển nhanh và đều cho đến hiện tại. Tại Hình 3 , có thể thấy sản lượng giai đoạn 2008 - 2010 bị hụt đi đáng kể. Điều này có thể xuất phát từ sự chưa đầy đủ của dữ liệu, hoặc cũng có thể là một hiện tượng đáng chú ý cần được quan sát thêm trong thời gian tới. 


\title{
Beitrag zum
}

\section{Typenproblem der Riemannschen Flächen *)}

\author{
Von Le-Van, Thiem, Zürich
}

\section{§ 1. Einleitung}

1. Jede einfach zusammenhängende, offene Riemannsche Fläche läßt sich bekanntlich eindeutig und konform auf einen Kreis $|z|<R \leqslant \infty$ abbilden. Je nachdem $R<\infty$ oder $R=\infty$ ist, heißt sie vom hyperbolischen oder vom parabolischen Typus. Im Anschluß an die Nevanlinnasche Wertverteilungslehre hat man speziell diejenigen Flächen $W_{q}$ behandelt, welche Überlagerungsflächen der Riemannschen Kugel sind und deren Windungspunkte nur über endlich vielen Grundpunkten liegen ${ }^{\mathbf{1}}$ ).

Hình 4. Công bố quốc tế đầu tiên (Le, 1947)

Về thể loại ấn phẩm, hầu hết các công trình được công bố ở dạng bài đăng tạp chí. Đây cũng là dạng ấn phẩm có tốc độ tăng ổn định nhất cho đến năm 2019. Riêng năm 2020, đã có 483 công bố được ghi nhận, trong khi những năm trước đều khoảng dưới 300 bài. Sự tăng đột ngột này là dấu hiệu cho thấy rằng nhiều bài báo chưa được ghi nhận trong thập kỉ trước đó.

\section{Publications Over Time}

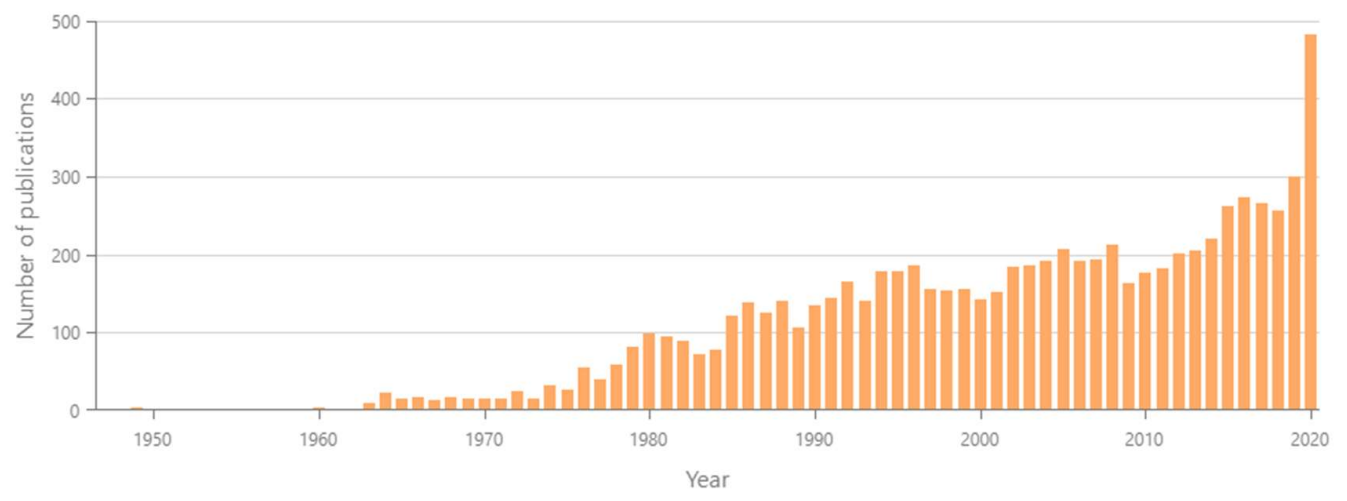

Hình 5. Số lượng bài Journal Article theo năm

Về sách, các tác giả Việt Nam đóng góp dưới hai dạng: chương sách hoặc cuốn hoàn chỉnh. Không phân biệt giữa hai loại, cơ sở dữ liệu ghi nhận 201 chương/cuốn sách tính tới thời điểm hiện tại. Dù tốc độ xuất bản không ổn định, nhưng hầu hết các năm đều có các ấn phẩm thuộc dạng sách. Giai đoạn 2007 đến 2013 là thời gian năng 
suất nhất về xuất bản sách, với ít nhất 6 ấn phẩm được xuất bản mỗi năm. Cuốn sách đầu tiên được xuất bản là "Lectures on the theory of symmetry of elementary particles. Part I. Theory of groups. Compact symmetry groups" của tác giả Nguyễn Văn Hiệu vào năm 1966. Đây là thời gian tác giả vẫn còn công tác tại Viện Nghiên cứu Hạt Nhân Dubna, Xô Viết. Ngoài ra, có hai cuốn sách được xuất bản bằng tiếng Việt là Phương trình đạo hàm riêng và Giải tích số. Cả hai cuốn đều được tác giả Nguyễn Minh Chương (Viện Toán học - VAST) chủ biên, và được ghi lại trên cơ sở dữ liệu của Zentralblatt MATH (ZbMath). Do hiện tại số lượng sách/ chương sách còn ít (có một số năm chỉ có 1 chương/ 1 cuốn), chúng tôi dự đoán số lượng thực có thể cao hơn đáng kể so con số được báo cáo này.

\section{Publications Over Time}

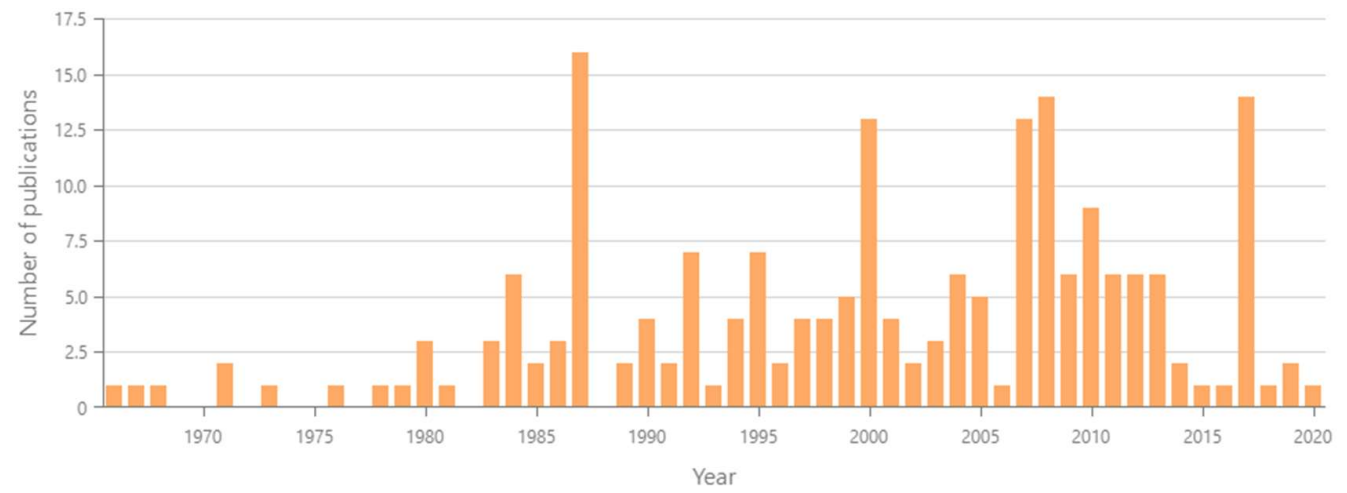

Hình 6. Số sách/ chương sách theo năm

Cho tới thời điểm hiện tại, cơ sở dữ liệu ghi lại 368 bài đăng hội thảo. Nhìn chung, số lượng bài đăng kỉ yếu không thay đổi nhiều qua các năm, trung bình sẽ có khoảng trên dưới 10 bài. Cũng có những năm số lượng bài hội thảo tăng đột biến (như 1995 và 2005 có khoảng 30 bài). Tuy nhiên, về tổng thể, số lượng bài hội thảo khá ổn định. 


\section{Publications Over Time}

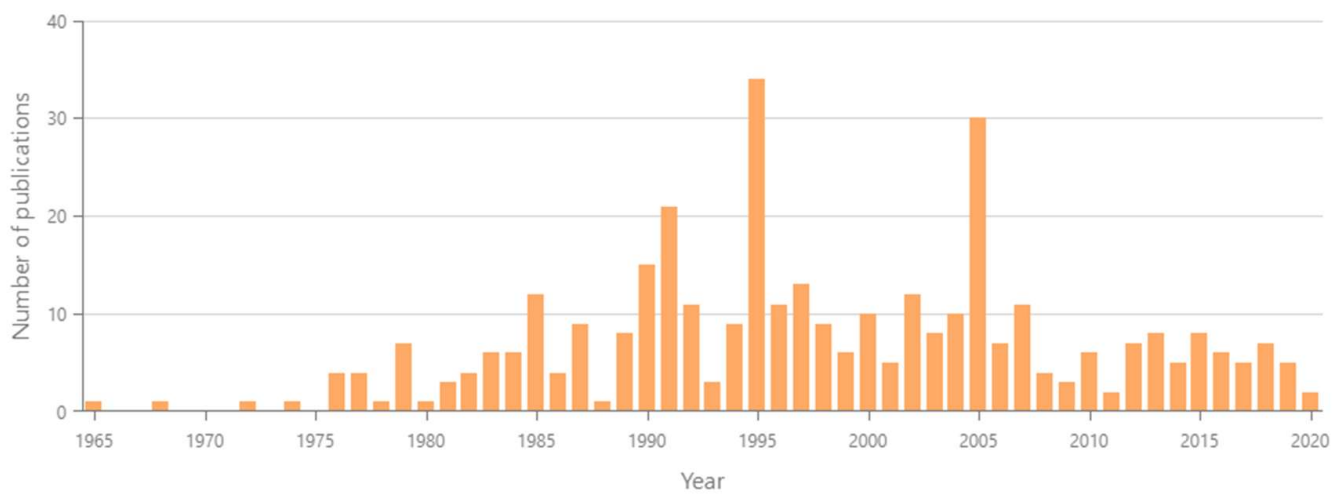

Hình 7. Số bài đăng hội thảo theo năm

\subsection{Số lượng tạp chí, sách, kỉ yếu}

Cơ sở dữ liệu đã ghi nhận 1267 nguồn. Trong đó, có 738 tạp chí, 148 cuốn sách, 281 kỉ yếu hội thảo. Về tạp chí, hiện nay 3 tạp chí được tác giả Việt Nam đăng bài nhiều nhất theo thứ tự bao gồm Acta Mathematica Vietnamica, Vietnam Journal of Mathematics, Journal of Optimization Theory and Applications (xem Bảng 1). Hai tạp chí trước có số lượng bài đăng vượt xa so với tạp chí thứ 3 . Điều này có thể do đây là hai tạp chí toán học quốc tế được cơ quan Việt Nam chủ quản nên được biết đến rộng rãi trong cộng đồng tác giả Việt Nam.

Bảng 1. 10 tạp chí nhiều bài nhất

\begin{tabular}{|c|c|c|c|}
\hline STT & Tên tạp chí & Số công bố & $\begin{array}{c}\text { Scimago } \\
\text { Quartile (2019) }\end{array}$ \\
\hline 1 & Acta Mathematica Vietnamica & 838 & Q3 \\
\hline 2 & Vietnam Journal of Mathematics & 695 & Q3 \\
\hline 3 & $\begin{array}{c}\text { Journal of Optimization Theory and } \\
\text { Applications }\end{array}$ & 158 & Q1/Q2 \\
\hline 4 & $\begin{array}{c}\text { Journal of Mathematical Analysis and } \\
\text { Applications }\end{array}$ & 157 & Q1/Q2 \\
\hline 5 & Journal of Algebra & 151 & Q1 \\
\hline 6 & Optimization & 135 & Q1/Q2 \\
\hline 7 & $\begin{array}{c}\text { Proceedings of the American Mathematical } \\
\text { Society }\end{array}$ & 113 & Q1 \\
\hline 8 & Nonlinear Analysis & 108 & Q1 \\
\hline 9 & Ukrainian Mathematical Journal & 91 & Q2 \\
\hline 10 & Journal of Global Optimization & 90 & Q1/Q2 \\
\hline
\end{tabular}


Các tác giả Việt Nam cũng đã ghi danh trên những tạp chí có ảnh hưởng nhất ngành toán. Dưới đây là danh sách một số tạp chí có hệ số Mathematical Citation Quotient (MCQ) cao nhất năm 2019 theo MathSciNet và số lượng bài được công bố bởi tác giả Việt Nam mà chúng tôi ghi nhận được (xem Bảng 2).

Bảng 2. Một số tạp chí toán học uy tín và số lượng công bố

\begin{tabular}{|c|c|c|c|}
\hline STT & Tạp chí & Số công bố & MCQ 2019 \\
\hline 1 & Annals of Mathematics & 13 & $5.24(97 \%$ cited $)$ \\
\hline 2 & Annals of PDE & 4 & $2.98(100 \%$ cited $)$ \\
\hline 3 & Journal of the American Mathematical Society & 2 & $5.14(99 \%$ cited $)$ \\
\hline 4 & Forum of Mathematics, Pi & 2 & $4.48(100 \%$ cited $)$ \\
\hline 5 & Acta Mathematica & 6 & $3.97(98 \%$ cited $)$ \\
\hline
\end{tabular}

Để rõ hơn về khả năng xuất bản của các tác giả Việt Nam, chúng tôi xem xét số lượng công bố theo thời gian trên 10 tạp trí uy tín nhất theo danh sách của đại học Copenhagen $^{1}$ (xem Hình 8). Tổng số bài báo được ghi nhập được là 8372 bài, trong đó chỉ 97 bài, tức khoảng $1 \%$ là được đăng trên tạp chí top 10. Vì vậy, số lượng công bố tăng đột biến trên hầu hết các tạp chí sau năm 2010 là nỗ lực không ngừng nghỉ để đạt đến đỉnh cao. Đây có thể là kết quả của hai sự kiện quan trọng đối với ngành toán Việt Nam vào năm 2010. Một là khi người Việt Nam đầu tiên, giáo sư Ngô Bảo Châu, đạt huy chương Fields danh giá. Hai là việc thành lập Chương trình Trọng điểm Quốc gia Phát triển Toán học. Một sự kiện là nguồn cảm hứng và động lực phấn đấu cho những người nghiên cứu toán học nhờ sự xuất hiện của một người Việt Nam đã thành công ghi danh trên bảng vàng toán học, một sự kiện là tạo môi trường, điều kiện phát triển ngành toán Việt Nam trên quy mô lớn. Thông tin chi tiết về các tác phẩm và tác giả công bố được trình bày tại Bảng $\mathrm{A}, \mathrm{B}$, và $\mathrm{C}$ phần Phụ lục 2 .

\footnotetext{
${ }^{1}$ Có thể truy cập tại: http://sym.math.ku.dk/about/internal/10-most-prestigiousnew.pdf
} 


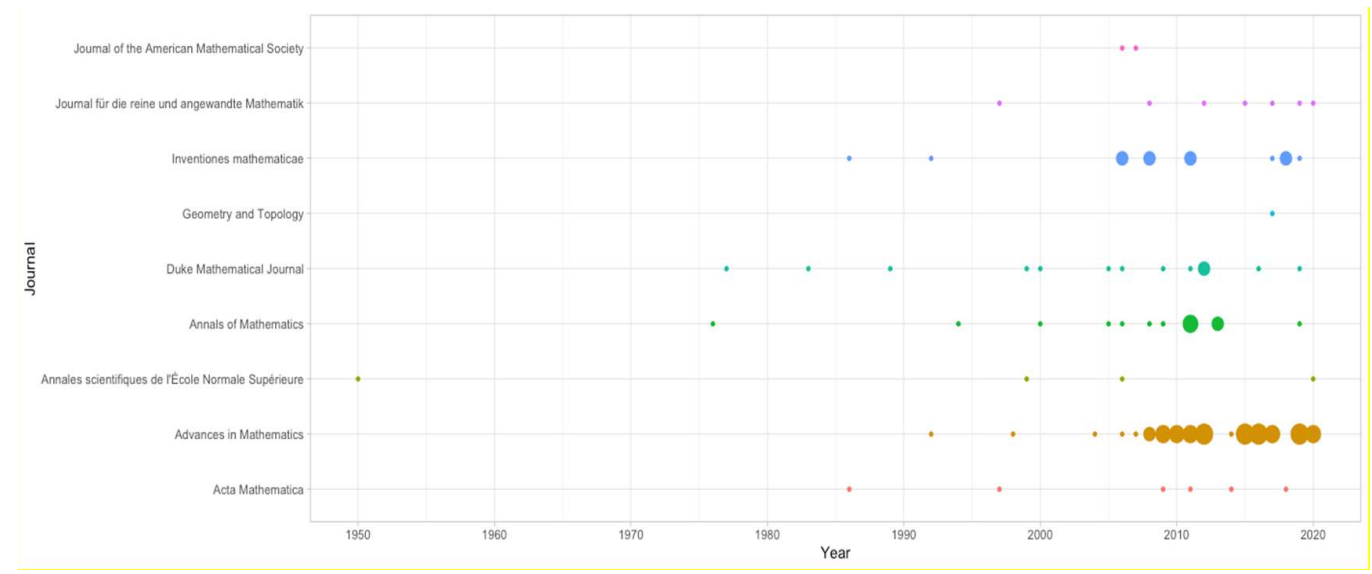

Hình 8. Số lượng công bố trên các tạp chí uy tin theo thời gian

Ngoài ra, cũng có sự thay đổi về quốc gia và ngôn ngữ của các tạp chí. Đây phần lớn là do sự thay đổi về nơi đào tạo, hợp tác của các tác giả cũng như xu hướng của thế giới. Thế hệ các nhà toán học ở giai đoạn đầu phần lớn được đào tạo và làm việc tại Liên Xô, họ có thể sử dụng thành thạo tiếng Nga và cũng đăng nhiều bài ở tạp chí tiếng Nga như Differentsial'nye Uravneniya (42 bài), Vestnik Moskovskogo Universiteta. Seriya 1. Matematika. Mekhanika (30 bài). Cũng có các công bố sử dụng tiếng Pháp, tiếng Đức và tiếng Anh. Trong khi đó, rất ít khi có thể thấy bài sử dụng các ngoại ngữ khác ngoài tiếng Anh trong các năm gần đây và cũng thấy sự vắng bóng của các tạp chí tiếng Nga.

\subsection{Số lượng tác giả}

Hiện nay, cơ sở dữ liệu đã có tổng cộng 3058 tác giả, trong đó 1566 tác giả Việt Nam và 1492 tác giả nước ngoài. Phân tích về tác giả cho thấy hai kết quả đáng chú ý. Thứ nhất, có xu hướng tăng về số lượng tác giả đăng bài và tác giả mới qua các năm. Thứ hai, số liệu cho thấy sự cải thiện khả năng nghiên cứu tác giả Việt Nam thông qua khả năng dẫn dắt và độc lập nghiên cứu.

Hình 9 thể hiện số lượng tác giả Việt Nam có xuất bản nghiên cứu theo các năm. Theo đó ta thấy được xu hướng tham gia nghiên cứu toán học ngày càng phổ biến tại 


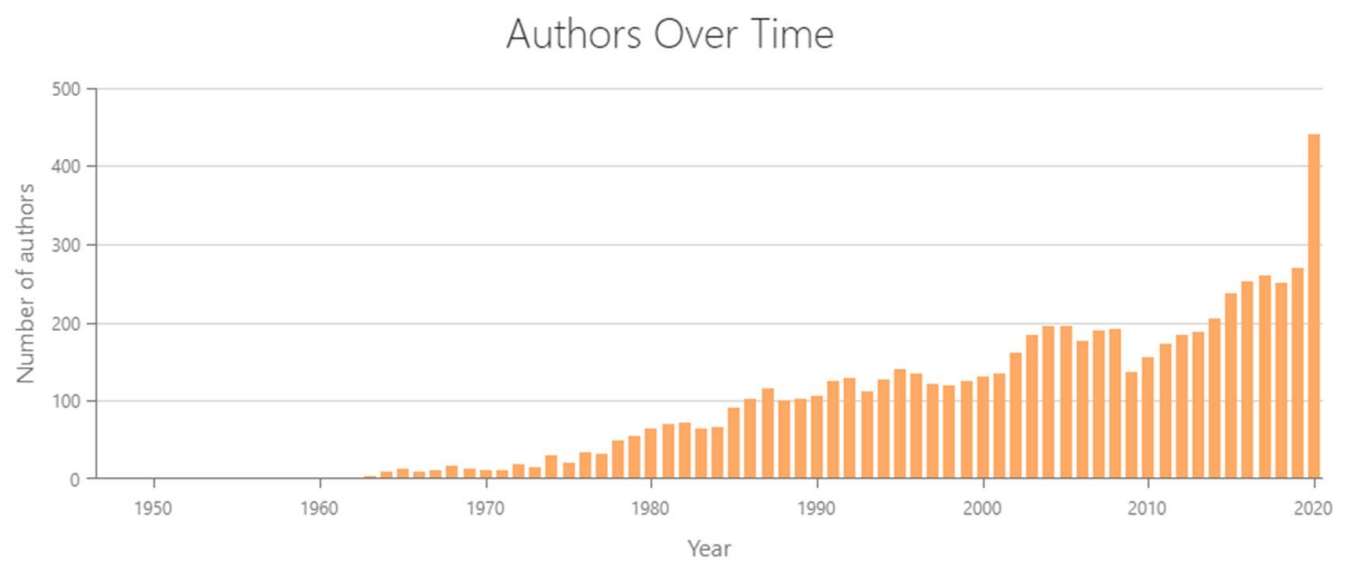

Hình 9. Số lượng tác giả Việt Nam theo năm

Khi phân tách năng suất xuất bản theo giới tính, ta thấy nam giới là thành phần chủ lực nghiên cứu toán học khi họ đóng góp phần lớn số lượng ấn phẩm hằng năm. Dù vậy, xu hướng tăng rõ rệt về số lượng bài có tác giả nữ cũng là tín hiệu tốt về khả năng đóng góp nghiên cứu của họ.

\section{Publications By Author's Gender}

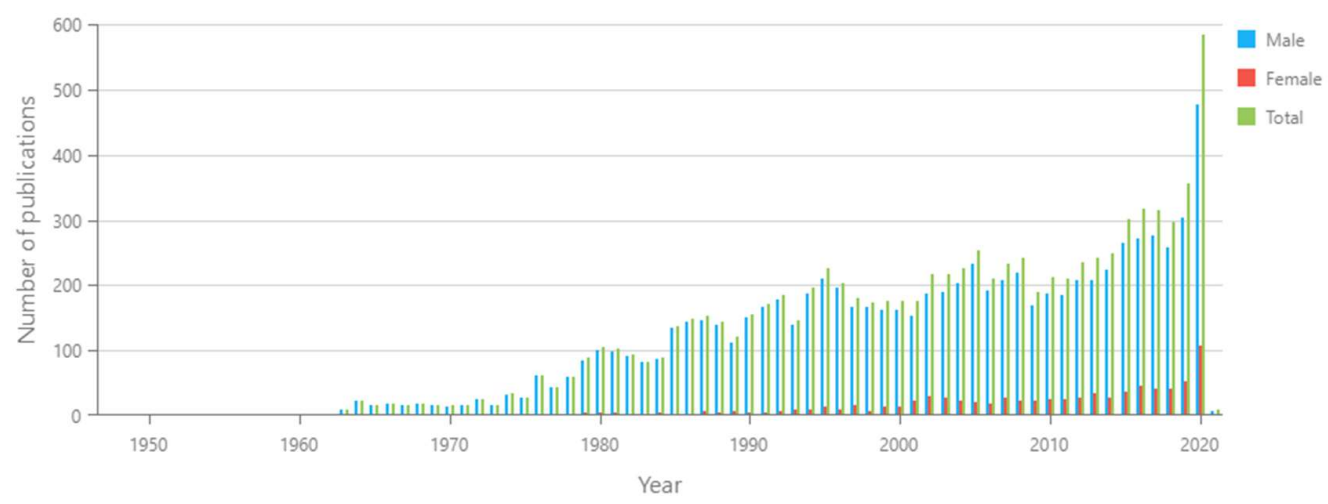

Hình 10. Số lượng bài nghiên cứu theo giới tính

Hình 11 về số lượng tác giả mới cũng cho kết quả tương tự. Dù số lượng tác giả mới tăng phần lớn là nhờ các tác giả nam, số liệu về nữ giới cho thấy sự cải thiện rõ rệt về giới trong toán học tại Việt Nam. Trong khi gần như không thấy xuất hiện thêm tác giả nữ vào giai đoạn trước 2000, số lượng tác giả mới là nữ tăng khá đều trong những

2 Ở đây, thời điểm tác giả được coi là mới tính theo ấn phẩm ra đời sớm nhất 
năm 2000 và tăng mạnh sau 2010 (xem Hình 10). Điều đáng chú ý là xu hướng này mới xuất hiện trong 20 năm gần đây dù lịch sử toán học nữ Việt Nam đã bắt đầu từ 1968 với bài báo "Behaviour of the diffraction peak for particles with arbitrary spins" của tác giả Nguyễn Thị Hồng trên tạp chí Nuclear Physics B (T. H. Nguyen, 1968). Bài báo trên được viết khi tác giả công tác tại Viện Nghiên cứu Hạt nhân Dubna, Xô Viết.

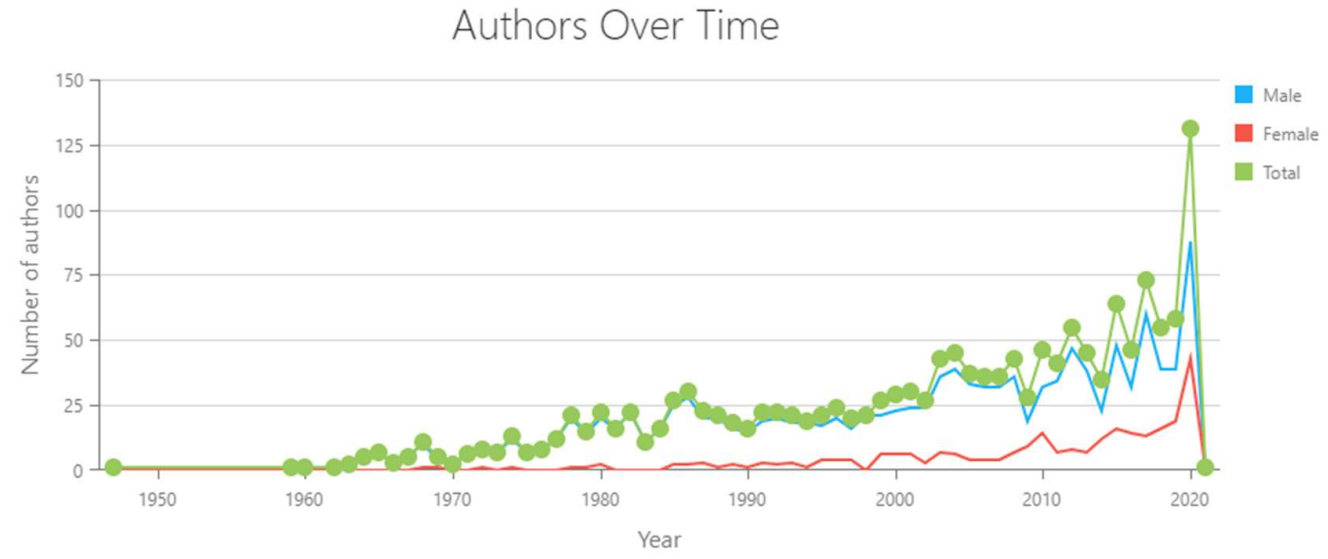

Hình 11. Số lượng tác giả nam và nữ mới theo năm

Ngoài số lượng tác giả, khả năng nghiên cứu thông qua việc dẫn dắt hoặc viết solo cũng là điểm đáng được quan tâm. Số lượng bài phân theo số lượng tác giả cho thấy toán học là ngành có xu hướng nghiên cứu độc lập hoặc theo nhóm nhỏ (xem Hình 12). Trong số 8,372 bài nghiên cứu được phân tích, khoảng 3,400 bài được viết solo, 3,000 bài được viết bới 2 tác giả.

\section{Publications By Number of Authors}

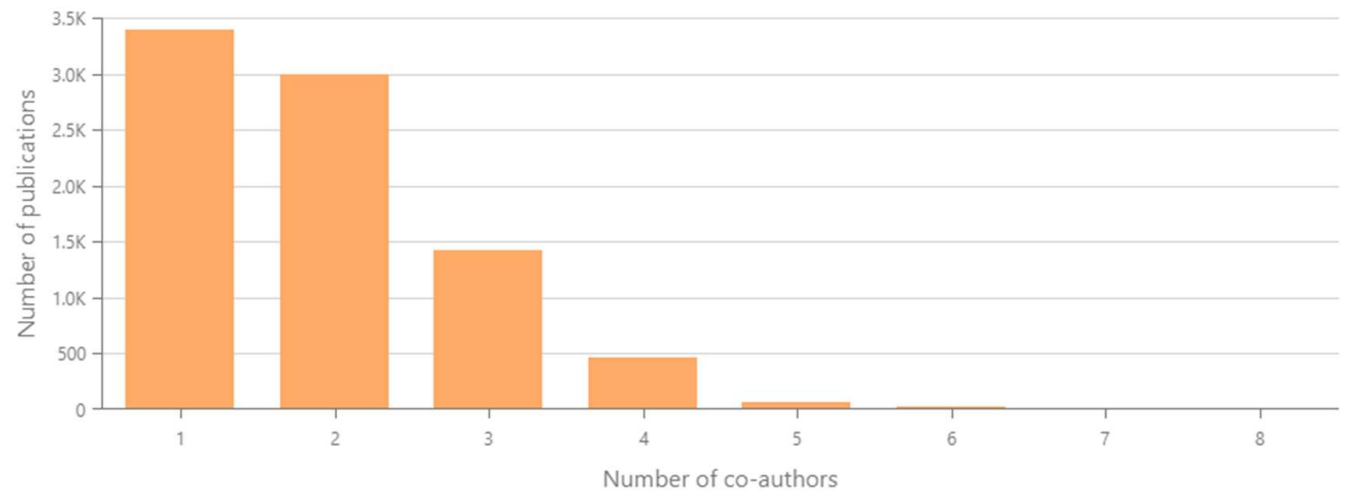

Hình 12. Số lượng bài nghiên cứu phân theo số lượng tác giả 
Biểu đồ số lượng tác giả dẫn dắt là người Việt Nam theo các năm cũng là dấu hiệu cho thấy sự cải thiện về khả năng nghiên cứu (xem Hình 13). Đối chiếu với Hình 8 (về số lượng tác giả Việt Nam hằng năm), ta thấy số tác giả từng đóng vai trò dẫn dắt nghiên cứu chiếm khoảng nửa số tác giả Việt Nam có đóng góp bài các năm.

\section{Lead Authors Over Time}

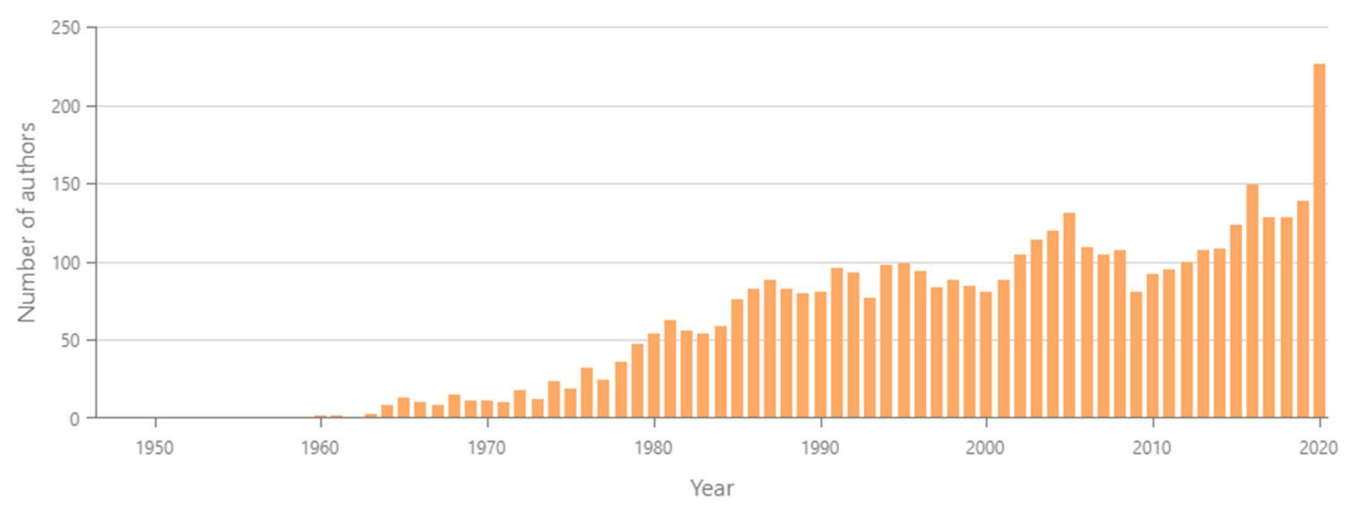

Hình 13. Số lượng tác giả dẫn dắt theo năm

Ngành toán có phân bố năng suất nghiên cứu tương đối lệch. Trong số 1566 tác giả Việt Nam, 10 tác giả có nhiều công bố nhất đã xuất bản 1232 bài, tương đương $14.3 \%$ tổng số lượng công bố. 100 tác giả năng suất cao nhất đã xuất bản 5261 bài (chiếm 62.4\%). Ngoài ra, trong số 100 tác giả trên, chỉ có 4 người là nữ với 140 bài báo. Bảng 3 cho thấy danh sách 5 tác giả có số lượng công bố nghiên cứu cao nhất (đây là danh sách tạm thời theo dữ liệu đang có).

Bảng 3. 5 tác giả Việt Nam có số lượng công bố cao nhất

\begin{tabular}{|c|c|c|c|}
\hline STT & Tác giả & Số lượng bài & Thời gian công bố \\
\hline 1 & Phạm Hữu Tiệp & 160 & $1986-2020$ \\
\hline 2 & Vũ Hà Văn & 144 & $1996-2020$ \\
\hline 3 & Vũ Ngọc Phát & 144 & $1980-2020$ \\
\hline 4 & Hoàng Tụy & 140 & $1964-2012$ \\
\hline 5 & Phan Quốc Khánh & 127 & $1979-2020$ \\
\hline
\end{tabular}

\subsection{Phân ngành}

CSDL SciMath sử dụng hệ thống phân ngành Mathematics Subject Classification System-MSC, hiện được các CSDL uy tín trong ngành như MathSciNet hay zbMATH sử dụng. Hiện nay CSDL SciMath ghi nhận 63 phân ngành MSC có đóng góp của các 
nhà Toán học Việt Nam. Bảng 4 trình bày đầy đủ 63 phân ngành theo số lượng công trình và tác giả.

Bảng 4. Số lượng công trình và tác giả theo từng phân ngành

\begin{tabular}{|c|c|c|}
\hline Phân ngành & $\begin{array}{c}\text { Số lượng công } \\
\text { trình }\end{array}$ & $\begin{array}{c}\text { Số lượng tác } \\
\text { giả }\end{array}$ \\
\hline General mathematics & 3 & 7 \\
\hline History and biography & 2 & 1 \\
\hline Mathematical logic and foundations & 13 & 22 \\
\hline Combinatorics & 147 & 123 \\
\hline Order, lattices, ordered algebraic structures & 26 & 27 \\
\hline General algebraic systems & 4 & 9 \\
\hline Number theory & 134 & 85 \\
\hline Field theory and polynomials & 22 & 18 \\
\hline Commutative algebra & 309 & 183 \\
\hline Algebraic geometry & 222 & 171 \\
\hline Linear and multilinear algebra; matrix theory & 56 & 60 \\
\hline Associative rings and algebras & 165 & 113 \\
\hline Nonassociative rings and algebras & 23 & 25 \\
\hline Category theory, homological algebra & 55 & 48 \\
\hline$\backslash(\mathrm{K} \backslash)$-theory & 12 & 8 \\
\hline Group theory and generalizations & 300 & 168 \\
\hline Topological groups, Lie groups & 36 & 36 \\
\hline Real functions & 162 & 152 \\
\hline Measure and integration & 27 & 32 \\
\hline Functions of a complex variable & 163 & 123 \\
\hline Potential theory & 37 & 48 \\
\hline Several complex variables and analytic spaces & 354 & 190 \\
\hline Special functions & 23 & 35 \\
\hline Ordinary differential equations & 323 & 271 \\
\hline Partial differential equations & 741 & 519 \\
\hline Dynamical systems and ergodic theory & 116 & 107 \\
\hline Difference and functional equations & 53 & 61 \\
\hline Sequences, series, summability & 4 & 9 \\
\hline Approximation and expansions & 63 & 66 \\
\hline Harmonic analysis on Euclidean spaces & 121 & 99 \\
\hline Abstract harmonic analysis & 11 & 18 \\
\hline Integral transforms, operational calculus & 42 & 44 \\
\hline Integral equations & 91 & 100 \\
\hline Functional analysis & 246 & 200 \\
\hline Operator theory & 472 & 380 \\
\hline $\begin{array}{c}\text { Calculus of variations and optimal control; } \\
\text { optimization }\end{array}$ & 489 & 331 \\
\hline Geometry & 11 & 22 \\
\hline Convex and discrete geometry & 72 & 70 \\
\hline
\end{tabular}




\begin{tabular}{|c|c|c|}
\hline Differential geometry & 76 & 71 \\
\hline General topology & 77 & 79 \\
\hline Algebraic topology & 67 & 57 \\
\hline Manifolds and cell complexes & 35 & 30 \\
\hline Global analysis, analysis on manifolds & 90 & 107 \\
\hline Probability theory and stochastic processes & 241 & 193 \\
\hline Statistics & 50 & 61 \\
\hline Numerical analysis & 348 & 385 \\
\hline Computer science & 73 & 95 \\
\hline Mechanics of particles and systems & 8 & 10 \\
\hline Mechanics of deformable solids & 32 & 41 \\
\hline Fluid mechanics & 69 & 83 \\
\hline Optics, electromagnetic theory & 43 & 40 \\
\hline Classical thermodynamics, heat transfer & 16 & 31 \\
\hline Quantum Theory & 44 & 26 \\
\hline Statistical mechanics, structure of matter & 19 & 24 \\
\hline Relativity and gravitational theory & 2 & 2 \\
\hline Geophysics & 3 & 6 \\
\hline Operations research, mathematical programming & 560 & 336 \\
\hline Game theory, economics, social and behavioral & 92 & 121 \\
\hline sciences & 44 & 67 \\
\hline Biology and other natural sciences & 191 & 144 \\
\hline Systems theory; control & 18 & 24 \\
\hline Information and communication, circuits & 3 & 4 \\
\hline Mathematics education & & \\
\hline & & 2 \\
\hline
\end{tabular}

Hiện nay, bảng phân ngành theo số lượng bài và tác giả này chỉ nên dừng ở mức độ tham khảo. Việc phân ngành ở đây được quy định theo từng bài chứ không xác định theo ngành chuyên môn của tác giả trên hồ sơ khoa học. Vì thế, một tác giả có thể có đóng góp trong nhiều hơn 1 ngành vì một bài không giới hạn số ngành. Bên cạnh đó, việc phân ngành ban đầu cũng gặp nhiều khó khăn do không có khả năng xác thực với các CSDL lớn như MathSciNet hay zbMath. Chúng tôi chỉ có thể phân ngành cho các công trình được ghi rõ ở bản PDF, hoặc có thể truy cập được miễn phí trên zbMath. Thời gian gần đây chúng tôi mới có thể truy cập vào MathSciNet để có thể cải thiện khả năng cập nhật dữ liệu này. Trong thời gian tới, chúng tôi sẽ tiếp tục bổ sung và chỉnh sửa để dữ liệu phân ngành được chính xác hơn.

\subsection{Về hợp tác}

Như đã đề cập, cơ sở dữ liệu đã có tổng cộng 3058 tác giả, trong đó 1566 tác giả Việt Nam và 1492 tác giả nước ngoài. Sự tương đương về số lượng tác giả trong và ngoài 
nước cho thấy các tác giả nước ngoài đã có vai trò lớn trong việc xây dựng và phát triển nền toán học Việt Nam.

Trong bản báo cáo sơ bộ này, chúng tôi sử dụng một phần hình ảnh về lưới quan hệ hợp tác giữa các tác giả trong giai đoạn 2000-2008 (Hình 14). Mỗi một tác giả là một điểm và đường thẳng nối giữa hai điểm thể hiện mối quan hệ hợp tác. Màu của các điểm thể hiện giới tính và quốc tịch tác giả: màu xanh biển là tác giả Việt Nam giới tính nam, màu xanh lá là người Việt giới tính nữ và màu vàng là tác giả nước ngoài. Độ lớn của các điểm phụ thuộc vào số lượng bài hợp tác.

Phân tích sơ bộ cho thấy các lưới quan hệ rộng rãi giữa các tác giả. Điều này thể hiện ở việc các nhóm con được liên kết với nhau. Ngoài ra, mối quan hệ càng lớn còn thể hiện các trường phái/ phân ngành nghiên cứu mạnh ở Việt Nam. Thí dụ như giữa nhóm của Hoàng Xuân Phú - Nguyễn Đông Yên - Nguyễn Định - Đinh Thế Lục Phan Quốc Khánh; hoặc nhóm của Ngô Việt Trung - Hà Huy Tài - Lê Tuấn Hoa.

Một số mạng lưới hiện độc lập với các mạng khác. Ví dụ như của tác giả Phạm Xuân Huyên hoặc của tác giả Đinh Văn Huỳnh. Có thể do hai tác giả này chỉ công tác ở nước ngoài trong giai đoạn 2000-2008 nên mạng lưới này chỉ gồm hoặc phần lớn là những tác giả nước ngoài và tách biệt với các nhóm khác. Ngoài ra, có một số tác giả solo như Lê Anh Vinh.

Dù vậy, trong các mạng lưới nghiên cứu, công bố vẫn thường được thực hiện bởi các nhóm nhỏ, phổ biến nhất là hai người hoặc 3 người. 


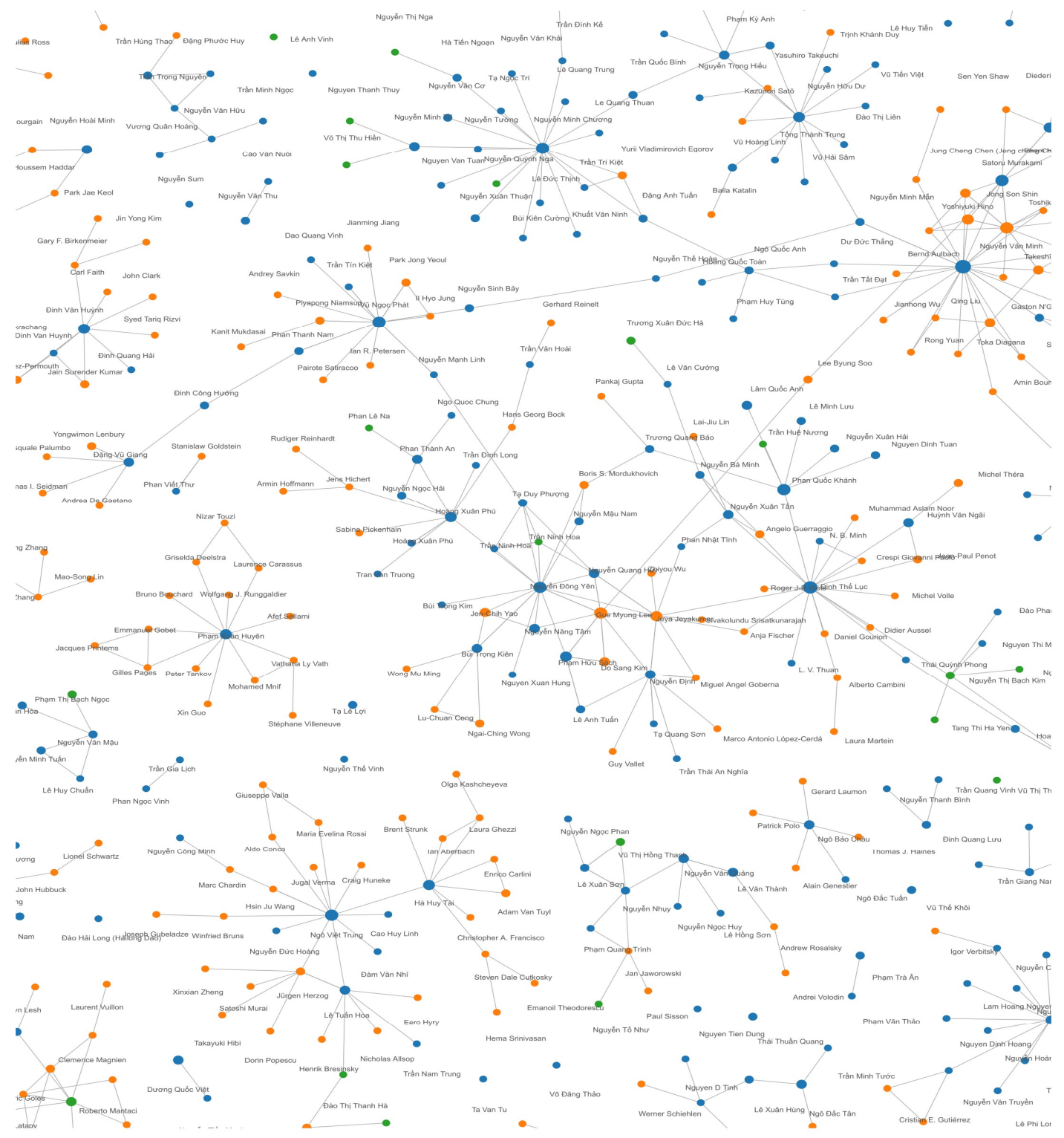

Hình 14. Mạng lưới hợp tác giai đoạn 2000-2008

Ngoài ra, để kiểm tra về tốc độ phát triển quan hệ hợp tác, chúng tôi sử dụng thêm mạng lưới hợp tác giai đoạn 1947-1960 và 1960-1970 (xem Hình 15 và Hình 16). Có thể thấy, so với mạng lưới hợp tác năm 2000-2008, hai giai đoạn 1947-1960 và 1960 - 1970 có mạng lưới nhỏ và đơn giản hơn rất nhiều. Sự thay đổi nhanh chóng giữa hai giai đoạn 1947-1960 và 1960-1970 cũng phản ánh xu hướng hợp tác xuất bản theo thời gian. 


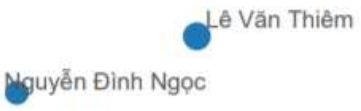

Qặng Đình Áng

Hình 15. Mạng lưới hợp tác giai đoạn 1947-1960

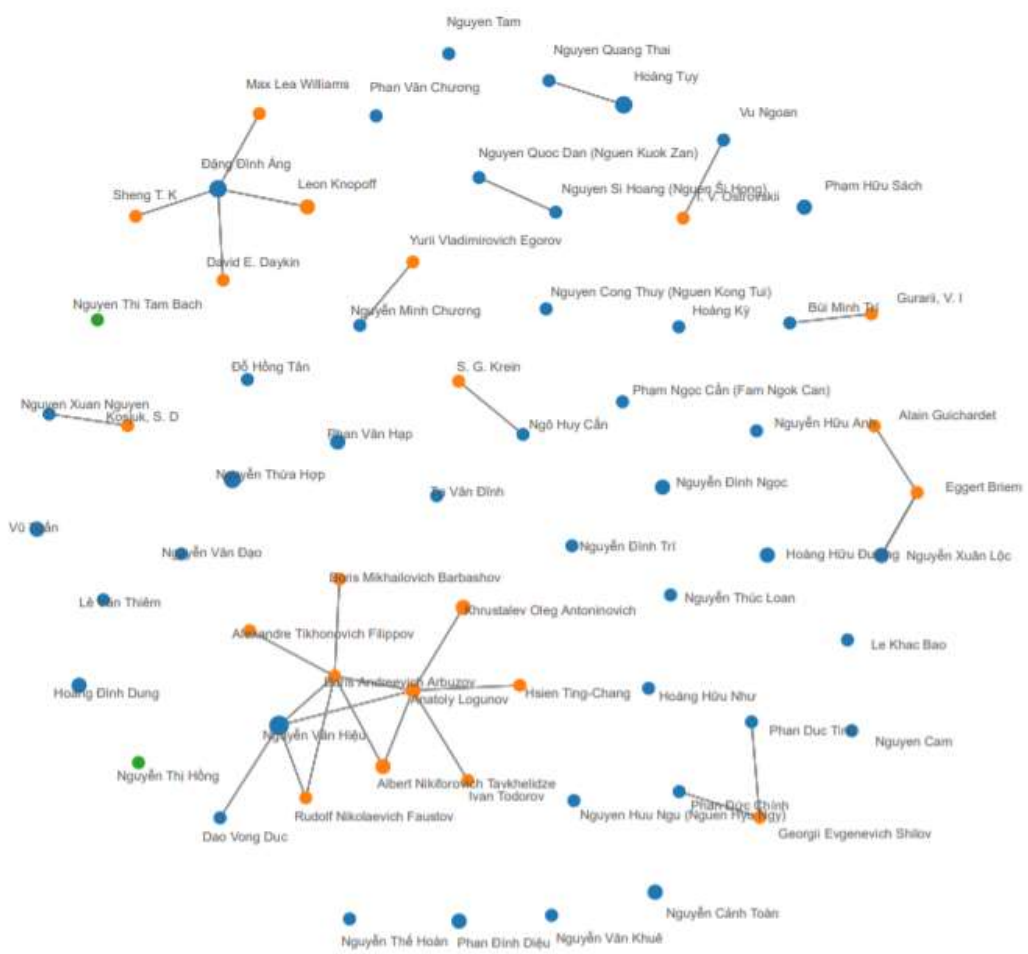

Hình 16. Mạng lưới hợp tác giai đoạn 1960-1970 


\subsection{Về cơ quan}

Tính từ 1947 đến nay, các cơ quan có số lượng xuất bản nổi bật đều là những cơ sở nghiên cứu về toán lâu đời tại Việt Nam. Nổi bật là Viện Toán học - VAST với 2696 ấn phẩm đã được ghi lại. Vị trí này có thể khó thay đổi trong thời gian ngắn vì lượng bài của Viện Toán đang gấp 4 lần so với đơn vị đứng thứ hai, Đại học khoa học tự nhiên - Đại học quốc gia Hà Nội. Bảng 5 là danh sách 5 cơ quan có số lượng công bố cao nhất (dựa trên trên dữ liệu đang có).

Bảng 5. 5 cơ quan có số lượng công bố cao nhất

\begin{tabular}{|c|c|c|}
\hline STT & Cơ quan & Số lượng công bố \\
\hline 1 & Viện Toán học - VAST & 2696 \\
\hline 2 & Đại học Khoa học Tự Nhiên - Đại học quốc gia Hà Nội & 596 \\
\hline 3 & Khoa Toán Tìn - Đại học sư phạm Hà Nội & 522 \\
\hline 4 & Đại học Khoa học Tự nhiên - Đại học quốc gia TP. HCM & 494 \\
\hline 5 & Viện Tin học - VAST & 239 \\
\hline
\end{tabular}

\section{Một số sự kiện quan trọng}

Toán học là ngành tiên phong trong xuất bản công bố quốc tế và đã thành công tạo hiệu ứng tràn về công bố quốc tế sang những ngành khác như công nghệ thông tin, vật lý ( $T$. T. H. Nguyen et al., 2020). Toán cũng là ngành ít ỏi ở Việt Nam có tiếng nói trong cộng đồng khoa học quốc tế và có những nhà khoa học nổi tiếng trên thế giới như Hoàng Tụy, Ngô Bảo Châu, Vũ Hà Văn. Với ngành đã có truyền thống lâu đời và vẻ vang như vậy, chúng tôi xin điểm lại một số sự kiện quan trọng và rất thú vị được thấy trong quá trình thu thập dữ liệu:

Năm 1947, nhà toán học Lê Văn Thiêm xuất bản công bố quốc tế đầu tiên, “Beitrag zum Typenproblem der Riemannschen Flächen” đăng trên Commentarii Mathematici Helvetici. Được coi là “cha đẻ” của ngành toán Việt Nam cùng với Hoàng Tụy, Lê Văn Thiêm đã có 13 công bố được xuất bản trên 7 tạp chí trong giai đoạn 19471981. Ông sử dụng đến bốn ngoại ngữ (tiếng Anh, tiếng Pháp, tiếng Nga và tiếng Đức), trong đó tiếng Pháp được sử dụng cho 8 bài báo.

Giáo sư Hoàng Tụy cho ra đời phương pháp “lát cắt Tụy” (Tuy’s cut) vào năm 1964, mở ra một chuyên ngành toán học mới: Lý thuyết tối ưu toàn cục. Sự nghiệp công bố toán học của ông trải dài 48 năm, từ 1964 đến 2012. Hoàng Tụy cũng là một trong những người có sự nghiệp đồ sộ nhất về mặt số lượng công bố với 140 công bố với đầy 
đủ thể loại: sách, bài báo và bài đăng hội thảo. Ông sử dụng cả tiếng Anh, tiếng Pháp và tiếng Nga.

Ngày 5/2/1969, Viện Toán học - VAST được thành lập. Trong lịch sử 51 năm của mình, Viện Toán đã trở thành đơn vị hàng đầu về công bố khoa học. Với 276 tác giả, Viện Toán đã có tổng cộng 2696 công bố đăng trên 585 nguồn khác nhau, đa dạng về thể loại và ngôn ngữ (xem Hình 17). Dựa trên phân ngành của các công bố, hệ thống dữ liệu cho thấy Operations research, mathematical programming (MSC 90); Calculus of variations and optimal control; optimization (MCS 49); Commutative algebra (MSC 13); và Algebraic geometry (MSC 14) có thể là ngành mạnh của viện. Các cán bộ nghiên cứu của viện không chỉ đóng góp nhiều về số lượng bài báo mà còn đăng bài ở nhiều tạp chí uy tín trên thế giới. Ví dụ, gần đây, hai cán bộ của Viện Toán học, Nguyễn Đăng Hợp và Ngô Việt Trung, đã có công bố "Depth functions of symbolic powers of homogeneous ideals" trên tạp chí Inventiones mathematicae (tạp chí có MCQ = 3.56, đứng thứ 7 năm 2019) (Nguyen and Ngo, 2019).

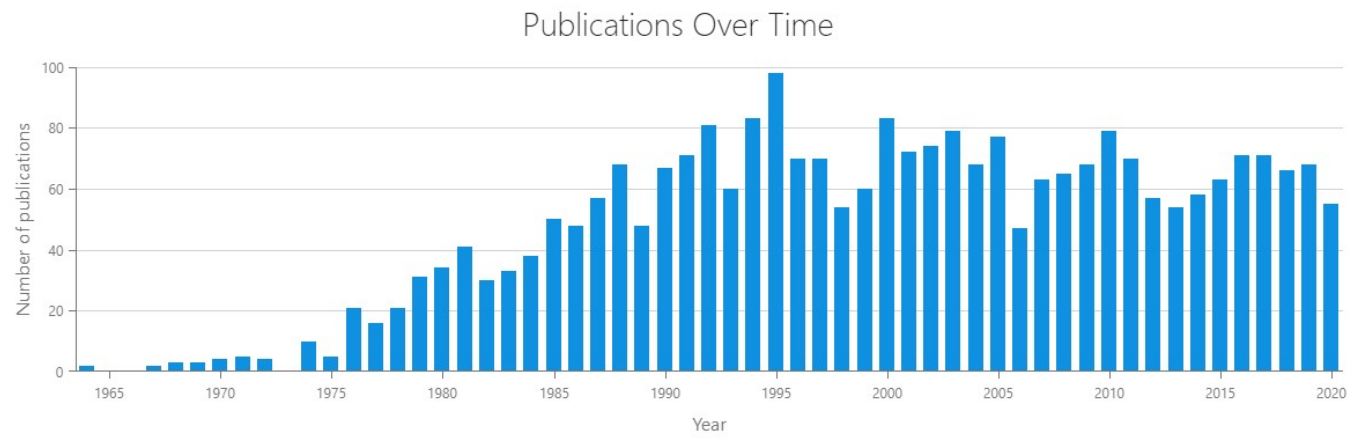

Hình 17. Số lượng công bố của Viện Toán học qua các năm

Năm 1976, tác giả Nguyễn Hữu Anh đăng công bố tựa đề "Lie Groups With Square Integrable Representations " trên Annals of Mathematics (Anh, 1976), một trong những tạp chí lâu đời và uy tín nhất ngành toán. Thú vị ở chỗ, cho đến nay, đây là tác giả duy nhất đăng bài trên tạp chí này có cơ quan làm việc tại Việt Nam (Đại học Bách Khoa Hà Nội). 


\section{Authors Over Time}

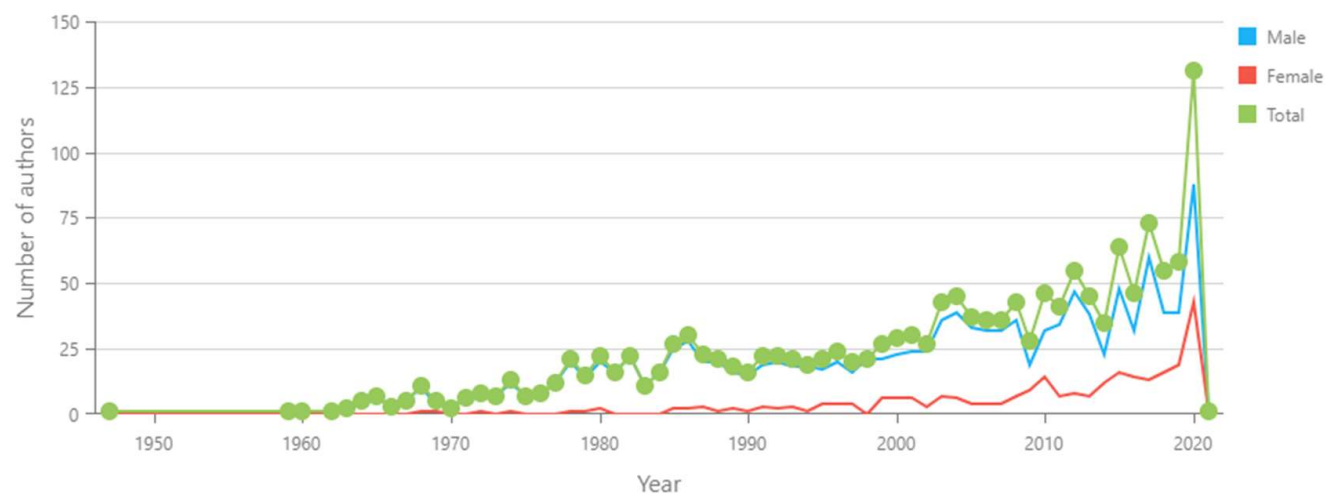

Hình 18. Số nhà nghiên cứu mới theo năm

Năm 2004, giáo sư Ngô Bảo Châu được trao giải thưởng Clay (Viện Toán học Clay) cùng với Gérard Laumon. Năm 2010, ông được trao Huy chương Fields, giải thưởng được coi là Nobel của Toán học. Giáo sư cũng là người Việt Nam duy nhất từng được nhận giải thương Clay và huy chương Fields tính tới thời điểm hiện tại. Ngoài ra giáo sư còn có các công trình nghiên cứu tiêu biểu khác như "Fibration de Hitchin et endoscopie", "Le lemme fondamental pour les algèbres de Lie", và "Le lemme fondamental pour les groupes unitaires" (Laumon \& Ngô, 2008; Ngô, 2006, 2010). Có lẽ thành quả vượt bậc của giáo sư Ngô Bảo Châu là nguồn cảm hứng cho rất nhiều nhà toán học trong nước, nhất là những nhà nghiên cứu trẻ. Sau năm 2010, có thể thấy số tác giả mới tăng nhiều (xem Hình 18), và dữ liệu cũng cho thấy tuổi trung bình và tuổi cao nhất của các nhà nghiên cứu mới giảm nhẹ (xem Hình 19).

\section{Age of Authors Over Time}

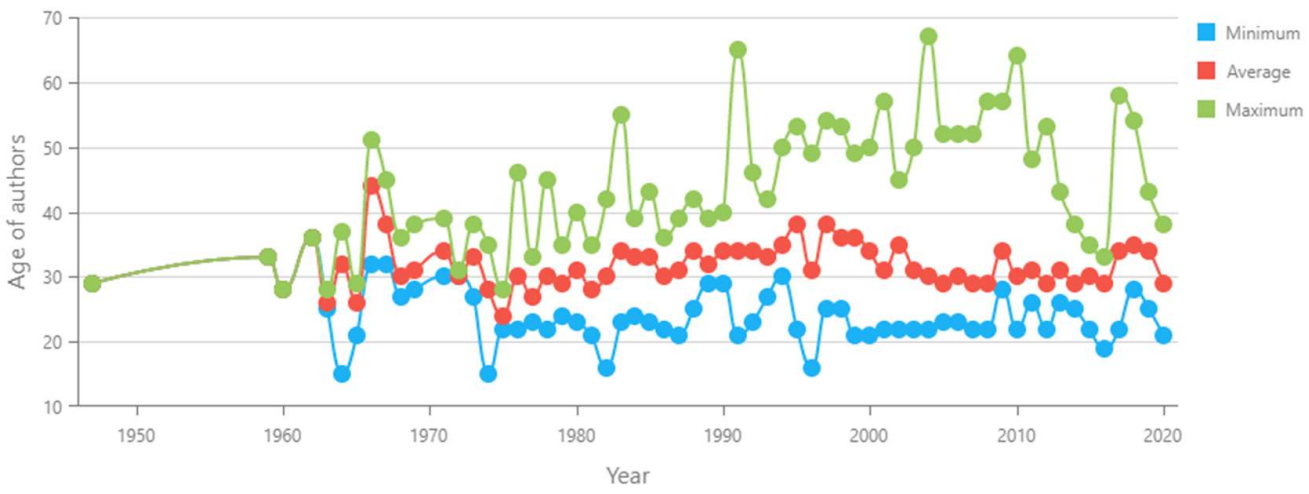

Hình 19. Tuổi của các nhà nghiên cứu mới theo năm 
Có thể nói, những thành công của nền Toán học Việt Nam ở hiện tại được xây dựng lên bằng nỗ lực và khát khao cống hiến không ngừng nghỉ của các thế hệ đi trước. Ngoài việc được đặt nền móng và xây dựng vững chắc bởi các tiền nhân như giáo sư Lê Văn Thiêm, giáo sư Nguyễn Thúc Hào, và giáo sư Hoàng Tụy, nền Toán học Việt Nam còn được hun đúc bởi sự khắc nghiệt của khói lửa chiến tranh qua hai cuộc kháng chiến chống Pháp và chống Mỹ. Tiêu biểu là quyển sách giáo khoa THPT về hình học cơ bản đầu tiên của giáo sư Hoàng Tuy. Quyển sách được giáo sư biên soạn trong điều kiện thiếu thốn tại chiến khu năm 1949 (Neal Koblitz \& Tuy, 1990).

Dường như sự khắc nghiệt của chiến tranh cũng không làm nao núng niềm đam mê tri thức cháy bỏng của các nhà Toán học Việt Nam, những người có thể chuyên tâm học hỏi, khám phá, và đàm luận Toán học giữa mưa bom bão đạn (Neal Koblitz, 2013). Nhờ vào những nhà Toán học "gan không núng, chí không mòn" mà Việt Nam - một đất nước lạc hậu về khoa học công nghệ, đạt được vị thế cân bằng với nước Mỹ - một siêu cường về khoa học công nghê, trên thế trận thông tin liên lạc và tình báo (Hiệu \& Koblitz, 2017).

Để có được những tư liệu quý báu về lịch sử của ngành Toán học Việt Nam, đóng góp của giáo sư Neal Koblitz (Đại Học Washington) là rất lớn. Nhờ vào các bài phỏng vấn và quan sát của ông đối với ngành Toán học Việt Nam (Neal Koblitz, 1979), kể từ khi còn non trẻ cho đến hiện tại, chúng ta mới có được cái nhìn rõ nét hơn về sự phát triển và vai trò của ngành Toán học qua các giai đoạn lịch sử của dân tộc. Gần đây nhất là buổi phỏng vấn của giáo sư Neal Koblitz với giáo sư Ngô Bảo Châu (Neal Koblitz, 2011). Nội dung của buổi phỏng vấn này không chỉ là nguồn thông tin quý báu mà còn là ngọn lửa đam mê truyền lại cho những nhà khoa học trẻ Việt Nam trong tương lai. Đây cũng là nguyện vọng của nhiều nhà nghiên cứu, nhà giáo trong lĩnh vực toán học, tiêu biểu và trực tiếp là GS Ngô Bảo Châu, người chủ trì dự án nghiên cứu dữ liệu này.

\section{4. Đánh giá chung và hướng phát triển tiếp theo}

CSDL SciMath bước đầu đã cho thấy những kết quả khả quan. Thông qua các thống kê của CSDL, một bức tranh toàn cảnh của ngành Toán học tại Việt Nam đã bắt đầu được hình thành. Trong tương lai, dữ liệu SciMath sẽ có thể đóng góp thêm nhiều công trình theo hướng nghiên cứu trắc lượng khoa học, đồng thời mở truy cập, tra cứu cho đại chúng. 
Hiện nay, vẫn còn một số vấn đề cần tiếp tục được giải quyết. Về mặt dữ liệu, nhóm dữ liệu dự kiến còn nhiều công trình chưa được ghi nhận trong CSDL SciMath. Với tính động của dữ liệu, chúng tôi dự kiến lượng dữ liệu ở mức đầy đủ cho toàn giai đoạn từ 1947 - 2020 sẽ là khoảng 10,000 công trình. Để đạt được con số này, nhóm dữ liệu sẽ tiếp tục lao động cật lực đến hết năm 2020. Sau đó, quá trình tiếp tục cập nhật dữ liệu cũng rất quan trọng.

Hiện nay, công tác thu thập dữ liệu chủ yếu dựa trên các trang thông tin có sẵn như Google Scholar, website của các nhà khoa học, trường đại học, cơ quan nghiên cứu, và các thông tin này được xác minh bởi các CSDL uy tín như MathSciNet, zbMath, Web of Science hay Scopus. Cố nhiều nguồn dữ liệu không được cập nhật thường xuyên, và một số tác giả không có trang cá nhân nên thông tin về tác giả hoặc công bố khó có thể cập nhật được vào CSDL. Bên cạnh đó, dữ liệu phục vụ cây phả hệ cũng cần được bổ sung. Khai thác dữ liệu đặc thù như dữ liệu cây phả hệ cần có sự đóng góp ý kiến chuyên gia trong ngành.

Về mặt tính năng, bên cạnh các số liệu được báo cáo ở trên, CSDL cần tiếp tục triển khai các phân tích khác như phân tích từng phân ngành, phân tích cây phả hệ, bản đồ hợp tác quốc tế/trong nước. Hiện nay, sự thiếu sót của các phân tích là do thiếu dữ liệu (trong trường hợp cây phả hệ) hoặc do phiên bản cuối cùng chưa được tinh chỉnh đầy đủ để phản ánh chính xác nhất (phân ngành, bản đồ hợp tác). Nhóm dữ liệu sẽ tiếp tục hoàn thiện các thiếu sót này trong thời gian tới.

Trong quá trình hoàn thiện chỉnh sửa CSDL, kết quả sơ bộ cũng cho thấy tiềm năng CSDL Scimath trở thành nguồn thông tin vô cùng hữu ích với cộng đồng học thuật và công chúng. Nhất là những người quan tâm tới toán học, $\mathrm{CSDL}$ cung cấp dữ liệu được thu thập có hệ thống và chất lượng để tham khảo về năng suất, khả năng và hợp tác trong nghiên cứu. Trong tương lai, có thể đây là bước đệm để thực hiện phân tích sâu hơn về ảnh hưởng của ngành toán thông qua lượt trích dẫn hoặc ảnh hưởng của tạp chí, đem lại góc nhìn sâu sắc hơn về lịch sử Toán học Việt Nam.

Kế hoạch tới của dự án bao gồm 3 mục tiêu chính: (1) tiếp tục tra cứu và cập nhật dữ liệu; (2) tiếp tục triển khai tích hợp các tính năng phân tích như cây phả hệ, mạng lưới nghiên cứu và (3) sử dụng CSDL sản xuất các sản phẩm phục vụ cộng đồng toán học và công chúng: sách chuyên khảo, công bố khoa học, và website tra cứu đại chúng (Vuong, 2018). Đặc biệt với mục tiêu số 3, chúng tôi dự kiến sẽ nhanh chóng sản 
xuất một cuốn sách chuyên khảo, tổng kết giai đoạn 75 năm phát triển của ngành Toán học Việt Nam.

Để hoàn thành các mục tiêu trên cần có bộ dữ liệu đầy đủ nhất và chất lượng nhất, nên chỉ riêng nỗ lực tìm kiếm của nhóm dữ liệu là chưa đủ. Vì vậy, chúng tôi mong nhận được sự giúp đỡ của các chuyên gia, các nhà nghiên cứu và cộng đồng toán học nói chung bằng cách đóng góp ý kiến và chia sẻ nội dung liên quan đến các công bố toán học, tác giả, cây phả hệ, cơ sở nghiên cứu. Đây chắc chắn sẽ là tài liệu quý báu để nhóm dữ liệu có thể cập nhật thông tin đầy đủ và chất lượng nhất cho CSDL cũng như các sản phẩm chính thức để phục vụ cộng đồng toán học và công chúng.

\section{Tài liệu tham khảo}

Anh, N. H. (1976). Lie Groups With Square Integrable Representations. Annals of Mathematics, 104(3), 431-458.

Hiệu, P. D., \& Koblitz, N. (2017). Cryptography during the French and American Wars in Vietnam. Cryptologia, 41(6), 491-511.

Koblitz, N. (1979). A mathematical visit to Hanoi. The Mathematical Intelligencer, $2(1), 38-42$.

Koblitz, N. (2011). Interview with Professor Ngô Bảo Châu. The Mathematical Intelligencer, 33(1), 46-50.

Koblitz, N. (2013). Grothendieck's 1967 Lectures in the Forest in Vietnam. The Mathematical Intelligencer, 35(2), 32-34.

Koblitz, N., \& Tuy, H. (1990). Recollections of mathematics in a country under siege. The Mathematical Intelligencer, 12(3), 16-34.

Laumon, G., \& Ngô, B. C. (2008). Le lemme fondamental pour les groupes unitaires. Annals of Mathematics, 169, 477-573.

Le, V. T. (1947). Beitrag zum Typenproblem der Riemannschen Flächen. Commentarii Mathematici Helvetici, 20(1), 270-287.

Ngô, B. C. (2006). Fibration de Hitchin et endoscopie. Inventiones mathematicae, 164(2), 399-453, doi: 10.1007/s00222-005-0483-7

Ngô, B. C. (2010). Le lemme fondamental pour les algebres de Lie. Publications Mathématiques de l'IHÉS, 111(1), 1-169.

Nguyen, H. D., \& Trung, N. V. (2019). Depth functions of symbolic powers of homogeneous ideals. Inventiones Mathematicae, 218(3), 779-827. 
Nguyen, T. H. (1968). Behaviour of the diffraction peak for particles with arbitrary spins. Nuclear Physics B, 8(2), 303-310.

Nguyen, T. T. H., Pham, H.-H., Vuong, Q.-H., Cao, Q.-T., Dinh, V.-H., \& Nguyen, D. D. (2020). The adoption of international publishing within Vietnamese academia from 1986 to 2020: A review. Learned Publishing, 33(4): EarlyView.

Vuong, Q. H. (2018). The (ir)rational consideration of the cost of science in transition economies. Nature Human Behaviour, 2(1), 5.

Vuong, Q. H. (2020). Reform retractions to make them more transparent. Nature, $582(7811), 149$.

Vuong, Q.-H., La, V.-P., Vuong, T.-T., Ho, M.-T., Nguyen, H.-K. T., Nguyen, V.-H., . . . Ho, M.-T. (2018). An open database of productivity in Vietnam's social sciences and humanities for public use. Scientific Data, 5(1), 180188. 


\section{Phụ lục 1}

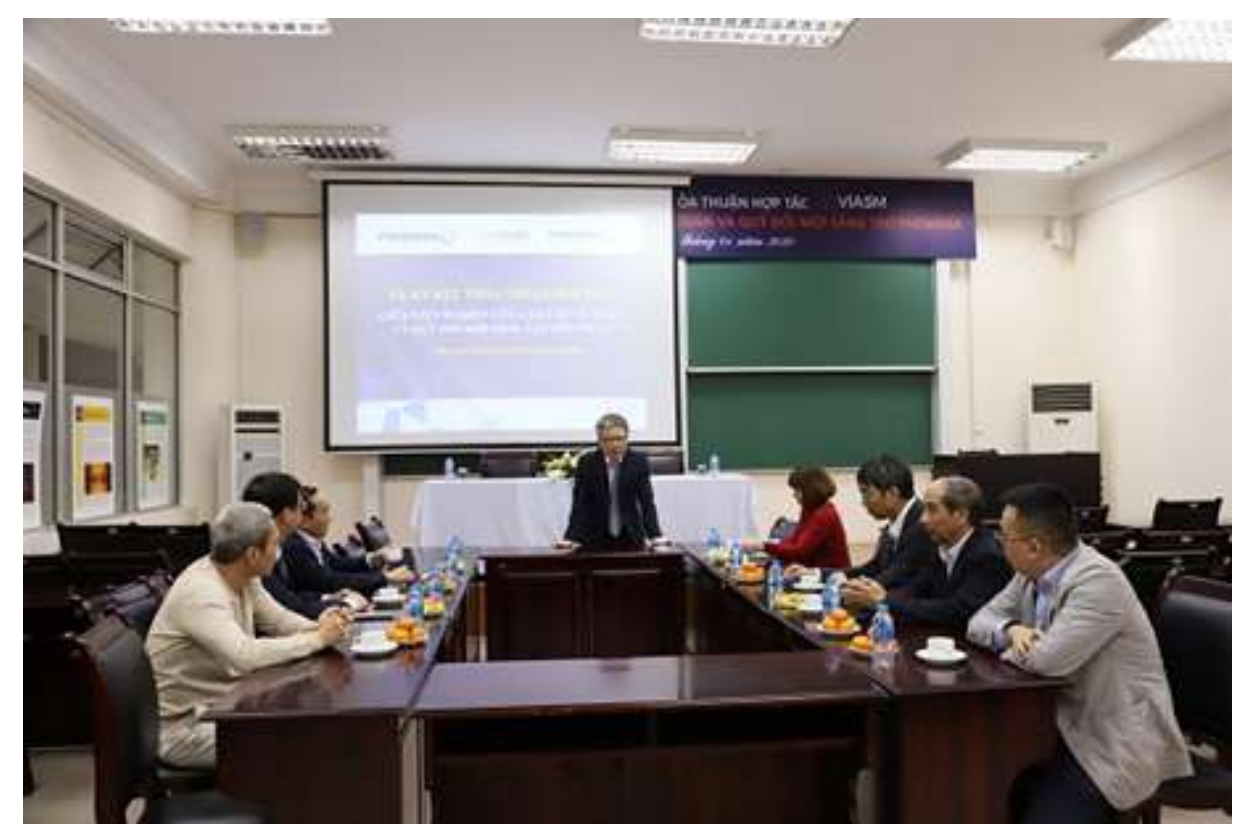

Hình A. Họp khởi động dự án (02/01/2020)

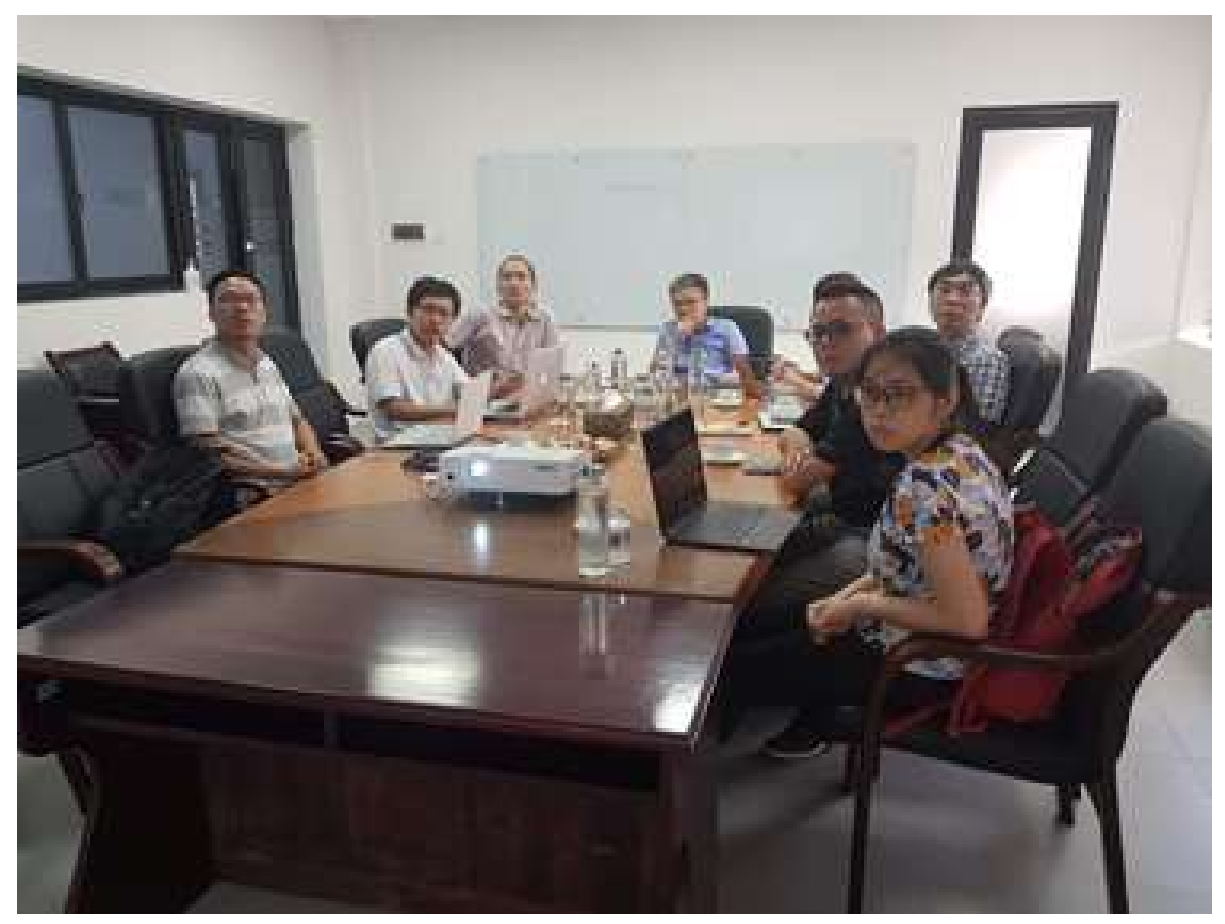

Hình B. Họp báo cáo dữ liệu lần thứ nhất (16/09/2020) 


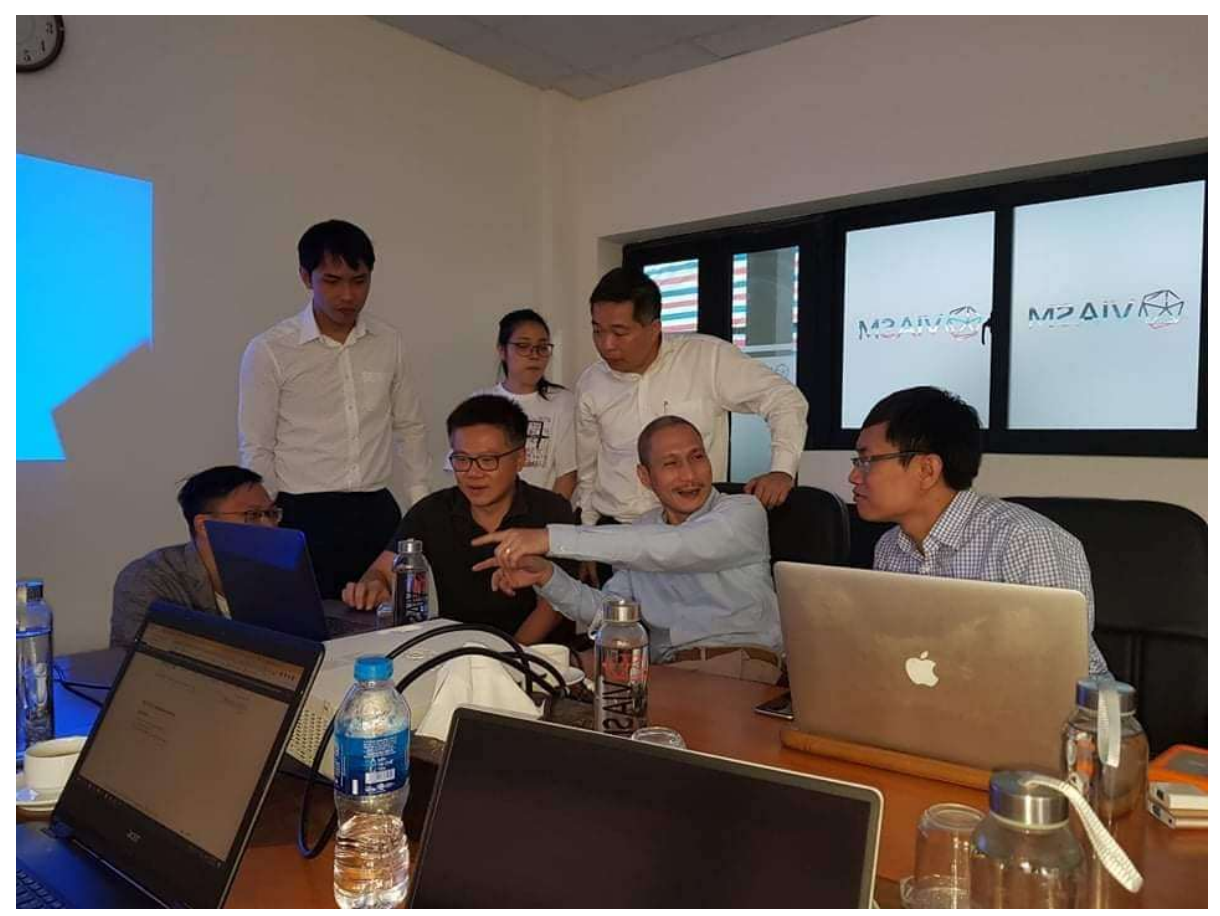

Hình C. Họp báo cáo dữ liệu lần thứ hai (26/10/2020) 
Phụ lục 2

Bảng A. Danh sách tác giả

\begin{tabular}{|c|c|c|c|c|}
\hline Họ và tên & Năm sinh & $\begin{array}{c}\text { Ngành tiêu } \\
\text { biểu }\end{array}$ & Số ấn phẩm & $\begin{array}{c}\text { Năm xuất bản đầu } \\
\text { tiên }\end{array}$ \\
\hline Alain Pham Ngoc Dinh & & $35 ; 65 ; 42$ & 45 & 1986 \\
\hline Bach Hung Khang & 1942 & $68 ; 62$ & 8 & 1974 \\
\hline Ban D. V & & & 1 & 1998 \\
\hline Banh Duc Dung & & 16 & 1 & 2008 \\
\hline Bui Cong Cuong & 1939 & $47 ; 91$ & 8 & 1976 \\
\hline Bui Dac Tac & 1950 & $32 ; 46$ & 9 & 1990 \\
\hline Bui Doan Khanh & 1942 & & 3 & 1995 \\
\hline Bui Duc Tien & 1963 & & 2 & 1992 \\
\hline Bui Huy Bach & & $35 ; 76 ; 93$ & 3 & 2018 \\
\hline Bui Khoi Dam & 1951 & 60 & 13 & 1981 \\
\hline Bui Kien Cuong & 1970 & $65 ; 35 ; 41$ & 3 & 2001 \\
\hline Bui Kim My & & 35 & 5 & 2016 \\
\hline Bui Le Trong Thanh & & & 1 & 2020 \\
\hline Bui Minh Phong & & & 1 & 1999 \\
\hline Bui Minh Tri & 1939 & & 9 & 1969 \\
\hline Bui Ngoc Muoi & & $90 ; 49$ & 1 & 2017 \\
\hline Bui Nguyen Thao Nguyen & 1989 & $32 ; 14$ & 2 & 2014 \\
\hline Bui Quang Nam & 1972 & 60 & 1 & 2017 \\
\hline Bui Quoc Hoan & & $32 ; 46$ & 5 & 2003 \\
\hline Bui Quoc Trung & 1985 & & 2 & 2017 \\
\hline Bui Tang Bao Ngoc & 1983 & & 1 & 2008 \\
\hline Bui Thanh Duy & & $35 ; 26 ; 65$ & 4 & 2015 \\
\hline Bui The Anh & 1980 & $93 ; 35 ; 42$ & 22 & 2006 \\
\hline Bui The Hung & 1983 & $90 ; 49 ; 91$ & 3 & 2011 \\
\hline Bui The Quan & 1978 & $93 ; 34 ; 35$ & 4 & 2011 \\
\hline Bui The Tam & 1953 & $90 ; 49$ & 10 & 1977 \\
\hline Bui Thi Hoa & & $90 ; 49$ & 2 & 2018 \\
\hline
\end{tabular}




\begin{tabular}{|c|c|c|c|c|}
\hline Bui Thi Kieu Oanh & & 32 & 1 & 2015 \\
\hline Bui Thu Lam & & & 3 & 2012 \\
\hline Bui Tien Dung & & $46 ; 34$ & 3 & 2000 \\
\hline Bui Trinh Khanh & & 30 & 1 & 2008 \\
\hline Bui Trong Kien & 1971 & $49 ; 47 ; 90$ & 36 & 2001 \\
\hline Bui Trong Kim & 1974 & $49 ; 47$ & 1 & 2001 \\
\hline Bui Tuong Tri & & & 2 & 1980 \\
\hline Bui Van Chien & & & 4 & 2013 \\
\hline Bui Van Dinh & & $90 ; 65 ; 49$ & 7 & 2011 \\
\hline Bui Van Thanh & & & 3 & 1990 \\
\hline Bui Viet Huong & 1984 & $65 ; 46 ; 35$ & 4 & 2010 \\
\hline Bui Vu Quang & 1980 & $60 ; 62$ & 1 & 2008 \\
\hline Bui Xuan Dieu & & $47 ; 34$ & 2 & 2015 \\
\hline Bui Xuan Hai & & $16 ; 20$ & 16 & 1989 \\
\hline Bui Xuan Quang & & $35 ; 34 ; 37$ & 3 & 2016 \\
\hline Buu Huu Thai & & $65 ; 90 ; 68$ & 2 & 2020 \\
\hline Can Van Hao & 1989 & $82 ; 05 ; 60$ & 10 & 2015 \\
\hline Can Van Tuat & & & 4 & 1984 \\
\hline \multicolumn{2}{|c|}{ Can Xuan Hien } & & 1 & 1973 \\
\hline Cao Hoang Tru & & & 2 & 2010 \\
\hline Cao Huu Hoa & & 35 & 2 & 2011 \\
\hline Cao Huy Linh & 1965 & $13 ; 14$ & 4 & 2005 \\
\hline Cao Thanh Tinh & & 34 & 1 & 2016 \\
\hline Cao Van Nuoi & 1970 & $60 ; 47 ; 43$ & 2 & 2002 \\
\hline Cao Xuan Phuong & & 62 & 4 & 2015 \\
\hline Chau Thi Ha & & $76 ; 80$ & 1 & 1989 \\
\hline Chu Binh Minh & & 93 & 1 & 2017 \\
\hline Chu Duc Khanh & & $74 ; 35$ & 3 & 1995 \\
\hline Chu Trong Thanh & & & 1 & 1997 \\
\hline Chu Van Dong & & 06 & 3 & 1985 \\
\hline
\end{tabular}




\begin{tabular}{|c|c|c|c|c|}
\hline Cung The Anh & 1977 & $35 ; 76 ; 37$ & 98 & 2004 \\
\hline D. N. Quynh & & $49 ; 90$ & 1 & 2001 \\
\hline D.V. Thanh (Do Van Thanh) & & 16 & 1 & 1991 \\
\hline Dam Van Nhi & & $13 ; 14$ & 4 & 1999 \\
\hline Dang Anh Tuan & 1981 & $26 ; 35 ; 30$ & 9 & 2003 \\
\hline Dang Dinh Ang & 1926 & $35 ; 45 ; 74$ & 90 & 1959 \\
\hline Dang Dinh Chau & 1953 & & 2 & 2005 \\
\hline Dang Dinh Hai & & $45 ; 47 ; 34$ & 14 & 1986 \\
\hline Dang Duc Trong & 1964 & $35 ; 65 ; 47$ & 67 & 1994 \\
\hline Dang Hai Long & 1988 & $49 ; 90 ; 65$ & 7 & 2018 \\
\hline Dang Hoa & & 90 & 2 & 2002 \\
\hline Dang Hung Thang & 1955 & $60 ; 47 ; 37$ & 36 & 1979 \\
\hline Dang Huu Dao & 1943 & & 9 & 1978 \\
\hline $\begin{array}{c}\text { Dang Huy Cuong (Cuong } \\
\text { Dang) }\end{array}$ & 1979 & & 1 & 2018 \\
\hline Dang Huy Ruan & 1939 & 68 & 5 & 1973 \\
\hline Dang Khai & & & 1 & 1985 \\
\hline Dang Khanh Hoi & 1948 & $58 ; 35$ & 6 & 1988 \\
\hline Dang Ngoc Hoang Thanh & & & 2 & 2020 \\
\hline Dang Phuoc Huy & & $60 ; 92$ & 3 & 2001 \\
\hline Dang Quang A & 1950 & $65 ; 35 ; 30$ & 16 & 1988 \\
\hline Dang Thanh $\mathrm{Ha}$ & 1968 & 68 & 1 & 2004 \\
\hline Dang Thanh Hai & & & 1 & 2005 \\
\hline Dang Thanh Son & & $35 ; 37 ; 76$ & 4 & 2013 \\
\hline Dang Thi My Van & & $90 ; 49 ; 65$ & 4 & 2009 \\
\hline Dang Thi Oanh & 1969 & 65 & 2 & 2017 \\
\hline Dang Thi Phuong Thanh & & $35 ; 45 ; 76$ & 7 & 2014 \\
\hline Dang Van Cuong & & $90 ; 49 ; 53$ & 6 & 2013 \\
\hline Dang Van Hieu & 1983 & $65 ; 47 ; 91$ & 19 & 2014 \\
\hline Dang Van Hung & 1950 & & 13 & 1979 \\
\hline Dang Vo Phuc & & 55 & 4 & 2015 \\
\hline
\end{tabular}




\begin{tabular}{|c|c|c|c|c|}
\hline Dang Vu Giang & 1965 & $42 ; 46 ; 26$ & 37 & 1991 \\
\hline Dang Vu Huyen & & & 1 & 1978 \\
\hline Dang Vu Phuong Ha & & 16 & 1 & 2010 \\
\hline Dang Xuan Cuong & & 54 & 1 & 2008 \\
\hline Dang Xuan Son & 1981 & $47 ; 65 ; 90$ & 2 & 2017 \\
\hline Danh Hua Quoc Nam & & $35 ; 47$ & 4 & 2019 \\
\hline Dao Bao Dung & & $35 ; 47$ & 1 & 2003 \\
\hline Dao Hai Long (Hailong Dao) & 1975 & $13 ; 14 ; 05$ & 43 & 2007 \\
\hline Dao Huu Ho & 1944 & 60 & 2 & 1994 \\
\hline Dao Huy Cuong & & $65 ; 76 ; 35$ & 2 & 2018 \\
\hline Dao Manh Khang & & 65 & 1 & 2020 \\
\hline Dao Ngoc Minh & & $90 ; 47 ; 49$ & 16 & 2015 \\
\hline Dao Nguyen Anh & & $65 ; 42 ; 43$ & 6 & 2017 \\
\hline Dao Nguyen Van Anh & & $20 ; 11$ & 2 & 2020 \\
\hline Dao Phan Vu & & 90 & 1 & 2006 \\
\hline Dao Phuong Bac & 1980 & $20 ; 14 ; 13$ & 7 & 2005 \\
\hline Dao Quang Khai & 1985 & $76 ; 35$ & 8 & 2013 \\
\hline Dao Quang Tuyen & & $60 ; 62$ & 7 & 1992 \\
\hline Dao Quang Vinh & & & 1 & 2007 \\
\hline Dao Thanh Tinh & 1962 & $35 ; 37$ & 3 & 1995 \\
\hline Dao Thi Lien & & & 1 & 2003 \\
\hline Dao Thi Thanh $\mathrm{Ha}$ & & $13 ; 14 ; 32$ & 3 & 2008 \\
\hline Dao Thi Thu Ha & & & 1 & 2014 \\
\hline Dao Trong Quyet & & $35 ; 37$ & 6 & 2012 \\
\hline Dao Trong Thi & 1951 & $49 ; 53 ; 58$ & 21 & 1978 \\
\hline Dao Tuan Anh & & 35 & 1 & 2020 \\
\hline Dao Van Duong & & $42 ; 47$ & 7 & 2013 \\
\hline Dao Van Tra & 1953 & $32 ; 22 ; 57$ & 5 & 1975 \\
\hline Dao Vong Duc & & & 9 & 1967 \\
\hline Dau Hoang Hung & 1979 & $32 ; 31 ; 41$ & 4 & 2005 \\
\hline
\end{tabular}




\begin{tabular}{|c|c|c|c|c|}
\hline Dau Hong Quan & & $30 ; 32$ & 1 & 2018 \\
\hline Dau Son Hoang & & & 1 & 2006 \\
\hline Dau The Cap & 1952 & & 5 & 1986 \\
\hline Dau The Phiet & & 32 & 1 & 2020 \\
\hline Dau Xuan Luong & & $47 ; 49 ; 65$ & 2 & 2010 \\
\hline Dinh Cao Duy Thien Vu & & 35 & 1 & 2011 \\
\hline Dinh Cong Huong & & $39 ; 92 ; 93$ & 14 & 2005 \\
\hline Dinh Dieu Hang & 1984 & $90 ; 49 ; 45$ & 5 & 2014 \\
\hline Dinh Duc Tai & & 16 & 1 & 2010 \\
\hline Dinh Dung & 1951 & $41 ; 42 ; 46$ & 72 & 1979 \\
\hline Dinh Hoang Anh & 1964 & & 1 & 1986 \\
\hline Dinh Huy Hoang & 1956 & $32 ; 46$ & 4 & 1996 \\
\hline Dinh Ngoc Quy & & $90 ; 49 ; 58$ & 5 & 2010 \\
\hline Dinh Ngoc Thanh & 1955 & $35 ; 45 ; 44$ & 20 & 1981 \\
\hline Dinh Nguyen Duy Hai & & $35 ; 65 ; 47$ & 10 & 2017 \\
\hline Dinh Nho Hao & 1960 & $65 ; 35 ; 49$ & 88 & 1987 \\
\hline Dinh Phu Bong & & & 5 & 1986 \\
\hline Dinh Quang Hai & 1979 & 16 & 4 & 2003 \\
\hline Dinh Quang Luu & 1947 & $60 ; 41 ; 91$ & 48 & 1980 \\
\hline Dinh Si Tiep & 1980 & $14 ; 32 ; 58$ & 10 & 2009 \\
\hline Dinh Sy Dai & 1948 & & 3 & 1987 \\
\hline Dinh Thanh Duc & 1960 & $44 ; 42 ; 46$ & 7 & 1993 \\
\hline Dinh Thanh Giang & 1988 & $52 ; 65 ; 68$ & 3 & 2010 \\
\hline Dinh Thanh Trung & & $14 ; 13$ & 1 & 2018 \\
\hline Dinh The Luc & 1952 & $90 ; 49 ; 52$ & 97 & 1978 \\
\hline Dinh Thi Ngoc Thanh & & & 2 & 1986 \\
\hline Dinh Tien Cuong & & $32 ; 37 ; 14$ & 32 & 2003 \\
\hline Dinh Trung Hoa & 1970 & $46 ; 15 ; 47$ & 10 & 2013 \\
\hline Dinh Van Huynh & 1950 & 16 & 89 & 1974 \\
\hline Dinh Van Ruy & & $65 ; 45$ & 5 & 1995 \\
\hline
\end{tabular}




\begin{tabular}{|c|c|c|c|c|}
\hline Dinh Xuan Khanh & & $35 ; 37 ; 34$ & 1 & 2016 \\
\hline Do Anh Tuan & & 11 & 1 & 2017 \\
\hline Do Ba Khang & 1958 & & 3 & 1982 \\
\hline Do Cong Khanh & 1949 & $34 ; 93 ; 47$ & 19 & 1982 \\
\hline Do Duc Dong & 1981 & & 1 & 2014 \\
\hline Do Duc Hung & & & 3 & 1987 \\
\hline Do Duc Tan & & $42 ; 35 ; 47$ & 3 & 2020 \\
\hline Do Duc Thai & 1961 & $32 ; 30 ; 46$ & 47 & 1991 \\
\hline Do Duc Thuan & & $34 ; 65 ; 47$ & 13 & 2008 \\
\hline Do Duy Chinh & & & 2 & 1982 \\
\hline Do Duy Hieu & & & 3 & 2013 \\
\hline Do Duy Thanh & & $65 ; 90$ & 1 & 2015 \\
\hline Do Hoai Vu & & $45 ; 47 ; 34$ & 1 & 2012 \\
\hline Do Hoang Giang & & $13 ; 14$ & 4 & 2010 \\
\hline Do Hoang Son & 1988 & $32 ; 35 ; 31$ & 8 & 2016 \\
\hline Do Hoang Viet & & $20 ; 05$ & 1 & 2020 \\
\hline Do Hong Nhat & & & 1 & 1988 \\
\hline Do Hong Tan & 1937 & $47 ; 54 ; 55$ & 29 & 1968 \\
\hline Do Huy Hoang & & 34 & 3 & 2016 \\
\hline Do Lan & & $35 ; 47 ; 34$ & 6 & 2010 \\
\hline Do Long Van & 1947 & $68 ; 94 ; 20$ & 39 & 1974 \\
\hline Do Ngoc Diep & 1950 & $22 ; 19 ; 46$ & 50 & 1974 \\
\hline Do Phi Nga & & 14 & 1 & 2001 \\
\hline Do Phuong An & & $32 ; 14 ; 30$ & 4 & 2013 \\
\hline Do Quang Yen & & $42 ; 37 ; 30$ & 15 & 2011 \\
\hline Do Tan $\mathrm{Si}$ & 1942 & & 1 & 1978 \\
\hline Do Thai Duong & 1994 & $31 ; 32$ & 3 & 2019 \\
\hline Do Thi Phuong Quynh & & $22 ; 11$ & 3 & 2015 \\
\hline Do Trong Hoang & 1985 & $13 ; 05$ & 7 & 2013 \\
\hline Do Van Kien & & 13 & 1 & 2018 \\
\hline
\end{tabular}




\begin{tabular}{|c|c|c|c|c|}
\hline Do Van Loi & & $47 ; 35 ; 34$ & 2 & 2016 \\
\hline Do Van Luu & 1944 & $90 ; 49 ; 26$ & 51 & 1982 \\
\hline Do Van Thuan & & 16 & 1 & 2020 \\
\hline Do Viet Bach & & & 1 & 2017 \\
\hline Do Viet Hung & 1981 & 57 & 1 & 2017 \\
\hline Do Xuan Duong & & 90 & 1 & 2003 \\
\hline Do Xuan Tho & & & 4 & 1985 \\
\hline Do Xuan Tung & 1983 & & 2 & 2017 \\
\hline Doan Cong Dinh & & $35 ; 30 ; 15$ & 3 & 2020 \\
\hline Doan Hai An & & & 1 & 1998 \\
\hline Doan Minh Luan & & $30 ; 47 ; 68$ & 6 & 2015 \\
\hline Doan Quang Manh & & $30 ; 11 ; 14$ & 4 & 2002 \\
\hline Doan Thai Son & 1984 & $37 ; 34 ; 26$ & 43 & 2007 \\
\hline Doan The Hieu & 1960 & $53 ; 49 ; 58$ & 9 & 1996 \\
\hline Doan Thi Thu Ha & & & 1 & 2015 \\
\hline Doan Trung Cuong & 1981 & $13 ; 14 ; 11$ & 13 & 2003 \\
\hline Doan Van Ngoc & & 35 & 3 & 1984 \\
\hline Du Duc Thang & 1975 & $47 ; 26$ & 2 & 2005 \\
\hline Du Thi Hoa Binh & 1987 & $47 ; 52 ; 15$ & 3 & 2014 \\
\hline Duc Ho & & $05 ; 13$ & 1 & 2015 \\
\hline Duong Anh Tuan & 1984 & $35 ; 34 ; 47$ & 7 & 2008 \\
\hline Duong Dang Xuan Thanh & 1982 & $34 ; 93 ; 47$ & 11 & 2006 \\
\hline Duong Hoang Dung & 1985 & 16 & 1 & 2014 \\
\hline Duong Hong Phong & 1953 & $81 ; 53 ; 32$ & 93 & 1977 \\
\hline Duong Luong Son & & & 1 & 1998 \\
\hline Duong Manh Hong & 1983 & $30 ; 35 ; 26$ & 2 & 2019 \\
\hline Duong Minh Duc & 1951 & $35 ; 47 ; 58$ & 35 & 1981 \\
\hline Duong Minh Thanh & & 17 & 1 & 2013 \\
\hline Duong Quang Hoa & & $46 ; 20 ; 22$ & 1 & 2010 \\
\hline Duong Quoc Huy & & $42 ; 47 ; 43$ & 4 & 2016 \\
\hline
\end{tabular}




\begin{tabular}{|c|c|c|c|c|}
\hline Duong Quoc Viet & 1954 & $13 ; 14$ & 15 & 1992 \\
\hline Duong Thi Anh Tuyet & & & 1 & 2015 \\
\hline Duong Thi Hong & & & 1 & 2019 \\
\hline Duong Thi Huong & 1983 & 13 & 2 & 2019 \\
\hline Duong Thi Kim Huyen & 1990 & $90 ; 49$ & 3 & 2016 \\
\hline Duong Thi Thanh Binh & & $35 ; 45 ; 65$ & 6 & 1997 \\
\hline Duong Thi Viet An & 1989 & $49 ; 90 ; 93$ & 6 & 2015 \\
\hline Duong Trong Luyen & 1984 & 35 & 8 & 2012 \\
\hline Duong Trong Nhan & & & 5 & 1980 \\
\hline Duong Viet Thong & & $47 ; 65 ; 68$ & 19 & 2012 \\
\hline Duong Xuan Thinh & & $35 ; 42$ & 3 & 2016 \\
\hline Duong Xuan Vinh & & $35 ; 76 ; 34$ & 2 & 2020 \\
\hline \multicolumn{2}{|c|}{ Giang Hoang (Hoang Ton Nu Huong Giang) } & & 1 & 2015 \\
\hline Ha Binh Minh & & 93 & 1 & 2017 \\
\hline Ha Binh Minh & & 34 & 1 & 2004 \\
\hline Ha Dai Ton & & $14 ; 22$ & 1 & 2009 \\
\hline Ha Dang Cao Tung & & & 1 & 1997 \\
\hline Ha Duc Vuong & & $46 ; 54$ & 3 & 2001 \\
\hline Ha Duy Hung & & $42 ; 47 ; 46$ & 12 & 2000 \\
\hline Ha Duyen Trung & & & 1 & 2012 \\
\hline Ha Hoang Hop & & & 2 & 1988 \\
\hline Ha Huong Giang & & $30 ; 32$ & 6 & 2012 \\
\hline Ha Huy Bang & 1960 & $46 ; 26 ; 42$ & 77 & 1982 \\
\hline Ha Huy Khoai & 1946 & $30 ; 11 ; 32$ & 38 & 1973 \\
\hline Ha Huy Tai & 1973 & $13 ; 05 ; 14$ & 51 & 1998 \\
\hline Ha Huy Vui & 1950 & $14 ; 32 ; 11$ & 46 & 1977 \\
\hline Ha Le Anh & & 51 & 2 & 1981 \\
\hline Ha Manh Linh & & $90 ; 49 ; 54$ & 1 & 2020 \\
\hline Ha Minh Hoang & & & 13 & 2015 \\
\hline Ha Minh Lam & 1979 & $13 ; 05 ; 14$ & 6 & 2007 \\
\hline
\end{tabular}




\begin{tabular}{|c|c|c|c|c|}
\hline Ha Minh Long & & & 1 & 2013 \\
\hline Ha Quang Minh & 1992 & & 1 & 2020 \\
\hline Ha Thi Ngoc Yen & 1980 & $39 ; 34 ; 35$ & 3 & 2004 \\
\hline Ha Thi Thanh Tam & 1985 & $47 ; 46 ; 03$ & 1 & 2017 \\
\hline Ha Thi Thu Hien & & 13 & 2 & 2015 \\
\hline Ha Tien Ngoan & 1951 & $35 ; 53 ; 47$ & 19 & 1977 \\
\hline Ha Tran Phuong & 1971 & $32 ; 30$ & 7 & 2007 \\
\hline Ha Vinh Tan & & & 3 & 1989 \\
\hline Ho Dac Nghia & 1970 & $35 ; 76$ & 1 & 2006 \\
\hline Ho Dang Phuc & 1955 & $60 ; 62$ & 15 & 1980 \\
\hline Ho Dinh Duan & 1947 & 16 & 2 & 1992 \\
\hline Ho Duc Viet & 1947 & & 4 & 1975 \\
\hline Ho Duy Binh & & & 1 & 2020 \\
\hline Ho Huu Viet & 1952 & & 3 & 1981 \\
\hline Ho Minh Toan & 1974 & $15 ; 46 ; 47$ & 10 & 2009 \\
\hline Ho Phi Tu & & $65 ; 90 ; 47$ & 1 & 2020 \\
\hline Ho Phu Quoc & & & 1 & 2014 \\
\hline Ho Si Tung Lam & & 35 & 1 & 2011 \\
\hline Ho Thuan & 1932 & $15 ; 65$ & 24 & 1971 \\
\hline Ho Thuc Quyen & & & 1 & 2011 \\
\hline Ho Tu Bao & & $68 ; 03$ & 7 & 2002 \\
\hline Ho Van Hoa & 1943 & & 2 & 1978 \\
\hline Ho Xuan Thang & 1973 & $13 ; 20 ; 18$ & 1 & 2003 \\
\hline Hoang Chi Thanh & 1952 & & 2 & 1991 \\
\hline Hoang Dinh Dung & 1922 & $30 ; 76 ; 86$ & 18 & 1967 \\
\hline Hoang Dung & & & 1 & 1988 \\
\hline Hoang Duong Tuan & 1964 & $90 ; 93 ; 49$ & 12 & 1992 \\
\hline Hoang Hai Hoc & & & 1 & 1979 \\
\hline Hoang Hoa Trai & & $93 ; 55$ & 2 & 2002 \\
\hline Hoang Hong Son & & & 5 & 1982 \\
\hline
\end{tabular}




\begin{tabular}{|c|c|c|c|c|}
\hline Hoang Huu Duong & 1936 & & 10 & 1964 \\
\hline Hoang Huu Nhu & 1932 & & 1 & 1968 \\
\hline Hoang Kiem & 1947 & & 4 & 1983 \\
\hline Hoang Ky & 1934 & & 4 & 1969 \\
\hline Hoang Le Minh & 1956 & $14 ; 81 ; 22$ & 7 & 1980 \\
\hline Hoang Le Truong & 1973 & 13 & 19 & 2008 \\
\hline Hoang Luu Cam Vu & & $35 ; 47$ & 1 & 2018 \\
\hline Hoang Mai Le & 1968 & 26 & 6 & 1998 \\
\hline Hoang Manh Tuan & & $65 ; 37$ & 1 & 2020 \\
\hline Hoang Manh Tuan & 1990 & $65 ; 37$ & 2 & 2020 \\
\hline Hoang Minh Chuong & & & 1 & 1985 \\
\hline Hoang Minh Hai & & & 4 & 1985 \\
\hline Hoang Nam & & $34 ; 47$ & 5 & 2003 \\
\hline Hoang Nam Dung & & $52 ; 51 ; 65$ & 2 & 2015 \\
\hline Hoang Ngoc Duong & & 65 & 1 & 2020 \\
\hline Hoang Ngoc Long & 1952 & & 1 & 1992 \\
\hline Hoang Ngoc Minh & & $11 ; 05 ; 37$ & 13 & 2012 \\
\hline Hoang Ngoc Tuan & & & 2 & 2013 \\
\hline Hoang Ngoc Yen & 1993 & 13 & 1 & 2019 \\
\hline Hoang Nhat Quy & 1979 & & 1 & 2013 \\
\hline Hoang Quang Tuyen & & 90 & 4 & 2000 \\
\hline Hoang Quoc Toan & 1945 & $35 ; 58$ & 12 & 1979 \\
\hline Hoang The Tuan & & $34 ; 26 ; 33$ & 21 & 2012 \\
\hline Hoang Thi Cam Thach & & $65 ; 90 ; 49$ & 1 & 2020 \\
\hline Hoang Thi Thao Phuong & 1987 & $65 ; 35 ; 74$ & 9 & 2013 \\
\hline Hoang Thi Vi & & & 1 & 1995 \\
\hline Hoang Tien (Hoang, T. H) & & & 5 & 1984 \\
\hline Hoang Tuy & 1927 & $90 ; 65 ; 78$ & 140 & 1964 \\
\hline Hoang Van Can & 1988 & 32 & 2 & 2018 \\
\hline Hoang Van Lai & & & 9 & 1973 \\
\hline
\end{tabular}




\begin{tabular}{|c|c|c|c|c|}
\hline Hoang Viet & & & 2 & 2016 \\
\hline Hoang Viet & & & 1 & 2012 \\
\hline Hoang Viet $\mathrm{Ha}$ & & 35 & 1 & 2020 \\
\hline Hoang Viet Long & & $03 ; 46 ; 47$ & 1 & 2017 \\
\hline Hoang Xuan Huan & 1954 & 53 & 4 & 1991 \\
\hline Hoang Xuan Phu & 1951 & $90 ; 52 ; 47$ & 67 & 1984 \\
\hline Hoang Xuan Sinh & 1933 & & 2 & 1978 \\
\hline Hong Ngoc Binh & & 13 & 1 & 2015 \\
\hline Huynh Ba Lan & 1956 & & 1 & 1993 \\
\hline Huynh Minh Hien & & $34 ; 37 ; 93$ & 1 & 2010 \\
\hline Huynh Mong Giao & & 26 & 1 & 2002 \\
\hline Huynh Mui & 1944 & & 7 & 1980 \\
\hline Huynh The Phung & 1959 & $52 ; 46 ; 90$ & 10 & 1989 \\
\hline Huynh Thi Hoang Dung & & $45 ; 47 ; 65$ & 1 & 2020 \\
\hline Huynh Thi Hong Diem & & $90 ; 49 ; 91$ & 5 & 2014 \\
\hline Huynh Thi Thanh Binh & & & 3 & 2012 \\
\hline Huynh Van Nam & 1978 & $03 ; 68 ; 62$ & 12 & 1999 \\
\hline Huynh Van Ngai & 1971 & $49 ; 90 ; 46$ & 22 & 1999 \\
\hline Huynh Viet Khanh & & 16 & 1 & 2020 \\
\hline Hy Duc Manh & & $90 ; 65 ; 49$ & 2 & 2020 \\
\hline Khu Quoc Anh & & 32 & 1 & 2004 \\
\hline Khuat Thi Binh & & $49 ; 47$ & 1 & 2020 \\
\hline Khuat Van Ninh & 1952 & $45 ; 47 ; 35$ & 6 & 1990 \\
\hline Khyng D. V & & & 1 & 1998 \\
\hline Kieu Huu Dung & & $47 ; 42$ & 1 & 2020 \\
\hline Kieu Phuong Chi & & $46 ; 32 ; 30$ & 5 & 2006 \\
\hline Kieu The Duc & & & 1 & 1982 \\
\hline Kieu Trung Thuy & & $65 ; 60$ & 1 & 2020 \\
\hline Kieu Van Hung & & $68 ; 94$ & 9 & 2004 \\
\hline L. V. Thuan & & & 1 & 2000 \\
\hline
\end{tabular}




\begin{tabular}{|c|c|c|c|c|}
\hline La Huu Chuong & & & 1 & 2012 \\
\hline La Thi Hong & & $47 ; 65 ; 91$ & 1 & 2020 \\
\hline Lam Hoang Nguyen & & & 1 & 2006 \\
\hline Lam Quoc Anh & 1974 & $90 ; 49 ; 91$ & 24 & 2004 \\
\hline Lam Quoc Dung & & & 1 & 2011 \\
\hline Le Anh Dung & & $47 ; 54 ; 53$ & 5 & 2003 \\
\hline Le Anh Minh & 1982 & 35 & 1 & 2020 \\
\hline Le Anh Tuan & 1963 & $49 ; 90 ; 54$ & 22 & 2003 \\
\hline Le Anh Tuan & & $60 ; 39 ; 34$ & 2 & 2018 \\
\hline Le Anh Vinh & 1983 & $05 ; 11 ; 52$ & 57 & 2007 \\
\hline Le Anh Vu & 1957 & $22 ; 46 ; 20$ & 7 & 1987 \\
\hline Le Anh $\mathrm{Vu}$ & & $68 ; 05$ & 1 & 2009 \\
\hline Le Ba Long & & $81 ; 46$ & 9 & 1990 \\
\hline Le Bich Phuong & 1984 & & 1 & 2016 \\
\hline Le Chi Ngoc & & & 1 & 2020 \\
\hline Le Cong Loi & 1972 & $39 ; 34 ; 65$ & 6 & 2001 \\
\hline Le Cong Nhan & & $34 ; 35$ & 4 & 2017 \\
\hline Le Cong Son (Shon, Le Khong) & & & 1 & 2007 \\
\hline Le Cong Thanh & 1951 & 68 & 7 & 1977 \\
\hline Le Cong Trinh & 1980 & $14 ; 15 ; 13$ & 11 & 2008 \\
\hline Le Dao Hai An & & $34 ; 92$ & 1 & 2017 \\
\hline Le Dinh Long & & $35 ; 47 ; 26$ & 18 & 2017 \\
\hline Le Duc Thang & & $35 ; 47$ & 4 & 2015 \\
\hline Le Duc Thinh & 1979 & & 1 & 2007 \\
\hline Le Duc Thoang & 1971 & 16 & 3 & 2002 \\
\hline Le Dung & & & 1 & 1989 \\
\hline Le Dung & & 35 & 6 & 2003 \\
\hline Le Dung Muu & 1949 & $90 ; 65 ; 47$ & 87 & 1978 \\
\hline Le Dung Trang & 1947 & & 1 & 1984 \\
\hline Le Gia Quoc Thong & & $47 ; 35$ & 1 & 2017 \\
\hline
\end{tabular}




\begin{tabular}{|c|c|c|c|c|}
\hline Le Giang & & $32 ; 35$ & 1 & 2020 \\
\hline Le H. An & 1978 & $49 ; 47$ & 1 & 2004 \\
\hline Le Hai An & 1978 & $35 ; 49 ; 58$ & 1 & 2005 \\
\hline Le Hai Khoi & 1960 & $47 ; 30 ; 32$ & 39 & 1983 \\
\hline Le Hai Yen & 1987 & $65 ; 90 ; 46$ & 9 & 2012 \\
\hline Le Hoan Hoa & 1947 & $45 ; 47 ; 34$ & 6 & 1979 \\
\hline Le Hoang Mai & & $18 ; 16$ & 1 & 2017 \\
\hline Le Hoang Tri & 1963 & $54 ; 57$ & 5 & 1994 \\
\hline Le Hong Lan & 1961 & & 3 & 1999 \\
\hline Le Hong Son & 1979 & & 1 & 2006 \\
\hline Le Hong Trang & 1983 & $52 ; 65 ; 68$ & 6 & 2012 \\
\hline Le Hong Van & 1962 & $51 ; 53$ & 2 & 1993 \\
\hline Le Hung Son & 1944 & $35 ; 30 ; 31$ & 35 & 1977 \\
\hline Le Hung Viet Bao & & & 1 & 2014 \\
\hline Le Huu Dien & & & 1 & 1985 \\
\hline Le Huy Chuan & 1978 & $45 ; 30$ & 2 & 2003 \\
\hline Le Huy Tien & 1977 & & 1 & 2007 \\
\hline Le Huy Vu & 1984 & & 1 & 2015 \\
\hline Le Huynh My Van & & 90 & 1 & 2020 \\
\hline Le Khac Bao & & & 1 & 1965 \\
\hline Le Khac Huynh & & 16 & 3 & 2004 \\
\hline Le Khanh Chau & 1958 & $54 ; 74$ & 15 & 1982 \\
\hline Le Khanh Luan & & 35 & 1 & 2011 \\
\hline Le Khoi Vy & 1959 & $35 ; 49 ; 74$ & 18 & 1990 \\
\hline Le Kim Luat & & & 1 & 1992 \\
\hline Le Ky Vy & & & 3 & 1992 \\
\hline Le Long Phi & & $35 ; 26 ; 46$ & 1 & 2019 \\
\hline Le Long Trieu & & $47 ; 46 ; 32$ & 32 & 2007 \\
\hline Le Manh Ha & 1979 & $68 ; 05 ; 91$ & 5 & 2009 \\
\hline Le Mau Hai & 1951 & $32 ; 46 ; 30$ & 65 & 1986 \\
\hline
\end{tabular}




\begin{tabular}{|c|c|c|c|c|}
\hline Le Minh Ha & 1972 & $55 ; 20 ; 18$ & 8 & 2001 \\
\hline Le Minh Hieu & 1986 & & 2 & 2020 \\
\hline Le Minh Luu & & $49 ; 90 ; 47$ & 10 & 2000 \\
\hline Le Minh Tri & & & 1 & 2005 \\
\hline Le Minh Triet & & $65 ; 35$ & 1 & 2018 \\
\hline Le Minh Tung & & $90 ; 26$ & 2 & 2002 \\
\hline Le Ngoc Lang & 1941 & & 7 & 1971 \\
\hline Le Ngoc Quynh & & $32 ; 30$ & 5 & 2012 \\
\hline $\begin{array}{c}\text { Le Ngoc Tuan (Le Ngok } \\
\text { T'euen) }\end{array}$ & & & 4 & 1982 \\
\hline Le Ngoc Xuan & 1977 & $65 ; 34$ & 3 & 2003 \\
\hline Le Nhat Huynh & & $35 ; 47 ; 65$ & 9 & 2019 \\
\hline Le Nhu Duong (Le N'y Dyong) & & & 2 & 1989 \\
\hline Le Nhu Hung & & & 1 & 1994 \\
\hline Le Phi Long & & $35 ; 49 ; 58$ & 1 & 2008 \\
\hline Le Phuoc Hai & & $90 ; 49$ & 2 & 2019 \\
\hline Le Phuong & & 35 & 2 & 2020 \\
\hline Le Phuong Quynh & & 35 & 1 & 2019 \\
\hline Le Quang Ham & 1982 & $11 ; 20$ & 3 & 2017 \\
\hline Le Quang Nam & 1980 & $74 ; 47 ; 65$ & 4 & 2003 \\
\hline Le Quang Ninh & & 32 & 1 & 2014 \\
\hline Le Quang Thuan & & & 1 & 2007 \\
\hline Le Quang Thuy & & $90 ; 49 ; 65$ & 11 & 2011 \\
\hline Le Quang Trung & & $47 ; 46 ; 58$ & 9 & 1985 \\
\hline Le Quoc Han & 1953 & & 2 & 1996 \\
\hline Le Quy Thuong & 1982 & $14 ; 32 ; 03$ & 3 & 2017 \\
\hline Le Si Dong & & 34 & 3 & 2012 \\
\hline Le Si Vinh & 1980 & & 1 & 2003 \\
\hline Le Sy Dong & & & 2 & 1987 \\
\hline Le Tai Thu & & 32 & 3 & 2002 \\
\hline Le Thai Thanh & & & 1 & 1993 \\
\hline
\end{tabular}




\begin{tabular}{|c|c|c|c|c|}
\hline Le Thanh & & & 1 & 1985 \\
\hline Le Thanh Hai & & & 1 & 1987 \\
\hline Le Thanh Hue & 1964 & $65 ; 90$ & 2 & 2009 \\
\hline Le Thanh Hung & & $41 ; 46$ & 1 & 2017 \\
\hline Le Thanh Manh & & & 1 & 1995 \\
\hline Le Thanh Quang & & 34 & 3 & 2008 \\
\hline Le Thanh Tung & & $90 ; 49 ; 46$ & 10 & 2011 \\
\hline Le Thi Diem Hang & & $35 ; 47$ & 1 & 2020 \\
\hline Le Thi Hoai An & 1958 & $90 ; 65 ; 49$ & 17 & 1996 \\
\hline Le Thi Hoai Thu & 1962 & 11 & 3 & 2003 \\
\hline Le Thi Hong Thom & & $30 ; 47$ & 1 & 2019 \\
\hline Le Thi Minh Duc & & $26 ; 35$ & 2 & 2020 \\
\hline Le Thi Ngoc Quynh & & & 1 & 2021 \\
\hline Le Thi Nhu Bich & & 42 & 1 & 2015 \\
\hline Le Thi Phuong Ngoc & 1966 & $35 ; 37 ; 65$ & 24 & 2006 \\
\hline $\begin{array}{c}\text { Le Thi Phuong Thuy (Le Thi } \\
\text { Thuy) }\end{array}$ & & $35 ; 37 ; 45$ & 9 & 2010 \\
\hline Le Thi Thanh & & 68 & 1 & 1994 \\
\hline Le Thi Thanh Nhan & 1970 & $13 ; 16$ & 21 & 1999 \\
\hline Le Thi Thu Giang & 1984 & $65 ; 35$ & 1 & 2018 \\
\hline Le Thi Thu Thuy & & $68 ; 62 ; 35$ & 2 & 2010 \\
\hline Le Thi Thu Thuy (UNCC) & & 35 & 1 & 2020 \\
\hline Le Thi Tuyet & & 35 & 2 & 2013 \\
\hline Le Thu Hoai & & 35 & 1 & 2006 \\
\hline Le Tien Nam & 1990 & & 2 & 2015 \\
\hline Le Tien Tam & & & 2 & 1984 \\
\hline Le Tien Vuong & & & 4 & 1973 \\
\hline Le Tran Tinh & & & 1 & 2018 \\
\hline Le Trieu Phong & & & 1 & 2004 \\
\hline Le Trong Lan & & $35 ; 20 ; 26$ & 2 & 2015 \\
\hline Le Trong Luc & 1952 & & 4 & 1991 \\
\hline
\end{tabular}




\begin{tabular}{|c|c|c|c|c|}
\hline Le Trung Hieu & & $05 ; 68$ & 1 & 2009 \\
\hline Le Trung Kien & & $68 ; 05$ & 1 & 2009 \\
\hline Le Trung Nghia & & $46 ; 26 ; 35$ & 1 & 2019 \\
\hline Le Tu Luc & 1971 & $90 ; 00$ & 4 & 1996 \\
\hline Le Tuan Hoa & 1957 & $13 ; 14 ; 05$ & 59 & 1986 \\
\hline Le Tung & & 65 & 1 & 2020 \\
\hline Le Tung & & 20 & 2 & 2013 \\
\hline Le Tung Son & 1979 & & 1 & 2007 \\
\hline Le Van Bao & & & 7 & 1984 \\
\hline Le Van Chong & & & 1 & 1986 \\
\hline Le Van Cuong & 1946 & & 2 & 2001 \\
\hline Le Van Dien & & & 2 & 1987 \\
\hline Le Van Dung & 1979 & 60 & 1 & 2010 \\
\hline Le Van Hap & 1957 & $35 ; 26 ; 46$ & 7 & 1988 \\
\hline Le Van Hien & 1979 & $34 ; 93 ; 92$ & 16 & 2008 \\
\hline Le Van Hien & & $90 ; 49$ & 3 & 2017 \\
\hline Le Van Hieu & 1975 & $35 ; 37 ; 34$ & 7 & 2010 \\
\hline Le Van Hoi & & & 1 & 2009 \\
\hline Le Van Hop & & & 2 & 1986 \\
\hline Le Van Hot & 1951 & & 2 & 1985 \\
\hline Le Van Thanh & 1943 & $32 ; 14$ & 12 & 1982 \\
\hline Le Van Thanh & 1979 & $60 ; 52$ & 16 & 2005 \\
\hline Le Van Thiem & 1918 & 30 & 13 & 1947 \\
\hline Le Van Thuyet & 1970 & 16 & 18 & 1991 \\
\hline Le Van Ut & & 35 & 1 & 2005 \\
\hline Le Van Vy & & $65 ; 47 ; 91$ & 2 & 2020 \\
\hline Le Viet Cuong & & 34 & 2 & 2019 \\
\hline Le Xuan Can (Suan Le Kan) & & & 2 & 1974 \\
\hline Le Xuan Dung & & 13 & 5 & 2012 \\
\hline Le Xuan Hung & & $05 ; 34 ; 60$ & 4 & 2003 \\
\hline
\end{tabular}




\begin{tabular}{|c|c|c|c|c|}
\hline Le Xuan Huy & & $33 ; 44 ; 45$ & 1 & 2013 \\
\hline Le Xuan Quang & & & 11 & 1971 \\
\hline Le Xuan Son & 1972 & $30 ; 26$ & 5 & 2003 \\
\hline Le Xuan Thanh & 1985 & $90 ; 47$ & 4 & 2016 \\
\hline Le Xuan Truong & 1978 & $35 ; 34 ; 42$ & 47 & 2007 \\
\hline Le Xuan Viet & & $93 ; 90$ & 1 & 2008 \\
\hline Lu Hoang Chinh & 1985 & $32 ; 53 ; 35$ & 20 & 2013 \\
\hline Luong Dang Ky & & $42 ; 46 ; 47$ & 37 & 2011 \\
\hline Luong Duc Trong & & $60 ; 65$ & 3 & 2017 \\
\hline Luong Thai Hung & & 35 & 2 & 2018 \\
\hline Luong The Dung & & & 1 & 2013 \\
\hline Luong Thi Tuyet & & $15 ; 30 ; 35$ & 2 & 2018 \\
\hline Luong Viet Chuong & & $14 ; 32$ & 1 & 2020 \\
\hline Luu Hoang Duc & 1981 & $37 ; 34 ; 35$ & 17 & 2003 \\
\hline Luu Hong Phong & & $35 ; 65$ & 1 & 2018 \\
\hline Luu Phuong Thao & & 13 & 1 & 2019 \\
\hline Luu Thi Hiep & & & 1 & 2019 \\
\hline Luu Vu Cam Hoan & & $35 ; 47 ; 74$ & 5 & 2017 \\
\hline Luu Xuan Thang & & $45 ; 65 ; 35$ & 1 & 2020 \\
\hline Luu Xuan Truong & & & 1 & 2019 \\
\hline Ly Kim Ha & & $32 ; 41 ; 42$ & 18 & 2014 \\
\hline Mach Nguyet Minh & & $35 ; 47 ; 42$ & 5 & 2009 \\
\hline Mai Thi Thu & & $26 ; 46 ; 45$ & 7 & 2000 \\
\hline Mai Anh Duc & 1975 & 32 & 3 & 2013 \\
\hline Mai Duc Thanh & 1973 & $35 ; 76 ; 65$ & 22 & 1995 \\
\hline Mai Hai An & 1980 & 41 & 2 & 2019 \\
\hline Mai Hoang Bien & 1982 & $16 ; 20 ; 05$ & 20 & 2012 \\
\hline Mai Thanh Nhat Truong & & $65 ; 35 ; 47$ & 2 & 2017 \\
\hline Mai Thi Kim Dung & & 35 & 1 & 2020 \\
\hline Mai Thi Ngoc Ha & & & 1 & 2020 \\
\hline
\end{tabular}




\begin{tabular}{|c|c|c|c|c|}
\hline Mai Van Tu & & $32 ; 11$ & 3 & 1995 \\
\hline Mai Viet Thuan & 1985 & $34 ; 93 ; 37$ & 12 & 2012 \\
\hline Mai Xuan Thao & 1957 & $41 ; 35 ; 42$ & 8 & 2002 \\
\hline My Vinh Quang & 1961 & 11 & 5 & 1987 \\
\hline N. B. Minh & & $49 ; 90$ & 2 & 2008 \\
\hline N. H. Loi & & $41 ; 40$ & 7 & 1985 \\
\hline N. M. Tuan & & & 3 & 1992 \\
\hline N.V. Loi & & $17 ; 16$ & 2 & 1985 \\
\hline Nghiem Do Quyen & & & 1 & 2008 \\
\hline Ngo Anh Tu & & & 4 & 1978 \\
\hline Ngo Anh Tuan & & 55 & 2 & 2019 \\
\hline Ngo Bao Chau & 1972 & $22 ; 14 ; 11$ & 26 & 1997 \\
\hline Ngo Dac Tan & 1952 & $05 ; 20$ & 41 & 1976 \\
\hline Ngo Dac Tuan & & 11 & 2 & 2008 \\
\hline Ngo Hoang Long & 1981 & $60 ; 65 ; 62$ & 6 & 2006 \\
\hline Ngo Huy Can & 1941 & $76 ; 41 ; 80$ & 19 & 1969 \\
\hline Ngo Lam Xuan Chau & & 34 & 1 & 2015 \\
\hline Ngo Manh Hung & & & 1 & 1991 \\
\hline Ngo Manh Tuong & & 65 & 1 & 2020 \\
\hline Ngo Minh Man & 1987 & $49 ; 91 ; 93$ & 1 & 2016 \\
\hline Ngo Quang Hung & & $05 ; 68 ; 94$ & 1 & 2003 \\
\hline Ngo Quoc Anh & 1978 & $35 ; 26 ; 46$ & 15 & 2005 \\
\hline Ngo Quoc Chung & & $34 ; 60$ & 1 & 2007 \\
\hline Ngo Quoc Hoan & & & 3 & 2015 \\
\hline Ngo Quy Dang & & $35 ; 34$ & 1 & 2018 \\
\hline Ngo Sy Tung & 1958 & 16 & 5 & 1994 \\
\hline Ngo Tan Phuc & 1985 & $18 ; 05 ; 16$ & 2 & 2017 \\
\hline Ngo Thi Hien & & $68 ; 94$ & 1 & 2018 \\
\hline Ngo Thi Ngoan & 1980 & $20 ; 11 ; 14$ & 6 & 2014 \\
\hline Ngo Thoi Nhan & 1986 & $65 ; 49 ; 39$ & 1 & 2017 \\
\hline
\end{tabular}




\begin{tabular}{|c|c|c|c|c|}
\hline Ngo Van Giang & & & 1 & 2019 \\
\hline Ngo Van Hoa & & $34 ; 47 ; 26$ & 17 & 2012 \\
\hline Ngo Van Luoc & & 30 & 18 & 1974 \\
\hline Ngo Viet Trung & 1953 & $13 ; 14 ; 05$ & 101 & 1978 \\
\hline Nguyen Ai Viet & 1952 & & 14 & 1983 \\
\hline Nguyen An Khuong & & 34 & 1 & 2015 \\
\hline Nguyen An Sum & & & 1 & 2004 \\
\hline Nguyen Anh Minh & & & 2 & 1978 \\
\hline Nguyen Anh Tam & & & 1 & 2010 \\
\hline Nguyen Anh Triet & & $35 ; 47 ; 65$ & 10 & 2016 \\
\hline Nguyen Anh Tuan & 1962 & & 3 & 1996 \\
\hline Nguyen Ba Minh & 1953 & $49 ; 90 ; 54$ & 15 & 2000 \\
\hline Nguyen Bac Van & & $62 ; 15$ & 7 & 1988 \\
\hline Nguyen Bao Tran & & $26 ; 46 ; 47$ & 2 & 2020 \\
\hline Nguyen Bich Huy & & $47 ; 35$ & 1 & 2018 \\
\hline Nguyen Bich Huy & 1956 & $47 ; 35 ; 34$ & 18 & 1980 \\
\hline Nguyen Bich Van & 1985 & $05 ; 15 ; 37$ & 3 & 2013 \\
\hline Nguyen Buong & 1949 & $47 ; 65 ; 49$ & 44 & 1985 \\
\hline Nguyen Cam & 1954 & $30 ; 35$ & 3 & 1995 \\
\hline Nguyen Cam & & & 1 & 1970 \\
\hline Nguyen Cang & & & 11 & 1972 \\
\hline Nguyen Canh Luong & 1961 & $35 ; 30$ & 5 & 1997 \\
\hline Nguyen Canh Nam & & $90 ; 65 ; 49$ & 3 & 2010 \\
\hline Nguyen Canh Toan & 1926 & & 7 & 1962 \\
\hline Nguyen Cao Menh & & & 4 & 1985 \\
\hline Nguyen Cao Tri & & $32 ; 34$ & 1 & 2020 \\
\hline Nguyen Cat Ho & 1941 & $68 ; 03 ; 90$ & 14 & 1972 \\
\hline Nguyen Chan & & $65 ; 34$ & 12 & 1980 \\
\hline Nguyen Chanh $\mathrm{Tu}$ & 1965 & $14 ; 32$ & 5 & 2001 \\
\hline Nguyen Chi Liem & 1971 & $39 ; 65 ; 34$ & 1 & 2013 \\
\hline
\end{tabular}




\begin{tabular}{|c|c|c|c|c|}
\hline Nguyen Chien & & 16 & 1 & 2003 \\
\hline Nguyen Chu Gia Vuong & & $22 ; 11$ & 2 & 2004 \\
\hline Nguyen Chuong & & & 1 & 1984 \\
\hline \multicolumn{2}{|c|}{ Nguyen Chuong Khue (Nguen Cyong Kue) } & & 2 & 1975 \\
\hline Nguyen Cong Dieu & & & 2 & 1993 \\
\hline Nguyen Cong Minh & 1980 & $13 ; 05$ & 10 & 2005 \\
\hline Nguyen Cong Phuc & & $35 ; 31 ; 42$ & 5 & 2006 \\
\hline Nguyen Cong Tam & & 35 & 3 & 1981 \\
\hline \multicolumn{2}{|c|}{ Nguyen Cong Thuy (Nguen Kong Tui) } & & 3 & 1965 \\
\hline Nguyen D Tinh & & $34 ; 35 ; 60$ & 1 & 2001 \\
\hline Nguyen Dac Liem & 1960 & 35 & 6 & 1994 \\
\hline Nguyen Dai Duong & 1985 & $14 ; 16 ; 13$ & 3 & 2017 \\
\hline Nguyen Dang Hanh & & & 1 & 1987 \\
\hline Nguyen Dang Ho Hai & 1981 & 20 & 2 & 2004 \\
\hline Nguyen Dang Hop & 1982 & $13 ; 14 ; 18$ & 12 & 2015 \\
\hline Nguyen Dang Minh & & $47 ; 35 ; 26$ & 9 & 2015 \\
\hline Nguyen Dang Phat & & & 1 & 1988 \\
\hline Nguyen Dang Tuan & 1958 & 34 & 4 & 1988 \\
\hline Nguyen Dang Tuyen & & & 1 & 2018 \\
\hline Nguyen Dinh & 1958 & $90 ; 49 ; 39$ & 55 & 1992 \\
\hline Nguyen Dinh Binh & & 35 & 9 & 2000 \\
\hline Nguyen Dinh Cong & 1960 & $34 ; 37 ; 60$ & 51 & 1984 \\
\hline Nguyen Dinh Dan & 1944 & $49 ; 90$ & 5 & 1979 \\
\hline Nguyen Dinh Hoa & & & 6 & 1984 \\
\hline Nguyen Dinh Hoang & & $90 ; 49$ & 2 & 2006 \\
\hline Nguyen Dinh Huy & 1956 & $39 ; 45 ; 93$ & 7 & 1990 \\
\hline Nguyen Dinh Lan & & $46 ; 32$ & 6 & 1998 \\
\hline Nguyen Dinh Liem & 1986 & $35 ; 78 ; 65$ & 22 & 2012 \\
\hline Nguyen Dinh Ngoc & 1932 & & 4 & 1960 \\
\hline Nguyen Dinh Phu & 1954 & $34 ; 47 ; 93$ & 31 & 2006 \\
\hline
\end{tabular}




\begin{tabular}{|c|c|c|c|c|}
\hline Nguyen Dinh Sang & & & 6 & 1980 \\
\hline Nguyen Dinh Thuc & & & 1 & 1997 \\
\hline Nguyen Dinh Tri & 1915 & 35 & 13 & 1966 \\
\hline Nguyen Dinh Tuan & & $49 ; 90 ; 47$ & 13 & 2006 \\
\hline Nguyen Doan Tien & & & 1 & 1982 \\
\hline Nguyen Doan Tuan & & $30 ; 32 ; 14$ & 10 & 1986 \\
\hline Nguyen Dong Anh & 1954 & $70 ; 60 ; 34$ & 44 & 1978 \\
\hline Nguyen Dong Phuong & & & 1 & 2016 \\
\hline Nguyen Dong Yen & 1959 & $49 ; 90 ; 26$ & 111 & 1985 \\
\hline Nguyen Du Vi Nhan & & $35 ; 26 ; 44$ & 1 & 2008 \\
\hline Nguyen Duc Dat & 1947 & 06 & 3 & 1996 \\
\hline Nguyen Duc Duyet & & 42 & 1 & 2020 \\
\hline Nguyen Duc Hau & & & 1 & 2008 \\
\hline Nguyen Duc Hien & 1976 & $65 ; 90 ; 49$ & 2 & 2014 \\
\hline Nguyen Duc Hieu & & & 5 & 1985 \\
\hline Nguyen Duc Hoang & & $13 ; 05 ; 14$ & 4 & 2001 \\
\hline Nguyen Duc Manh & 1982 & & 2 & 2018 \\
\hline Nguyen Duc Minh & 1963 & $13 ; 14 ; 16$ & 10 & 1992 \\
\hline Nguyen Duc Nghia & 1951 & 90 & 9 & 1982 \\
\hline Nguyen Duc Phuong & & $35 ; 26 ; 47$ & 9 & 2019 \\
\hline Nguyen Duc Tam & & 13 & 1 & 2010 \\
\hline Nguyen Duc Tuan & & & 5 & 1988 \\
\hline Nguyen Dung & & $65 ; 44 ; 35$ & 3 & 2005 \\
\hline Nguyen Duong Toan & & $35 ; 45 ; 76$ & 11 & 2012 \\
\hline Nguyen Duy Binh & & & 1 & 1997 \\
\hline Nguyen Duy Cuong & & $49 ; 90$ & 4 & 2020 \\
\hline Nguyen Duy Phuong & 1978 & $52 ; 51 ; 05$ & 3 & 2017 \\
\hline Nguyen Duy Tan & 1981 & $12 ; 20 ; 11$ & 20 & 2004 \\
\hline Nguyen Duy Thai Son & 1963 & $35 ; 26 ; 58$ & 17 & 1988 \\
\hline Nguyen Duy Thanh & & $47 ; 49 ; 35$ & 2 & 2002 \\
\hline
\end{tabular}




\begin{tabular}{|c|c|c|c|c|}
\hline Nguyen Duy Thinh & 1995 & & 1 & 2020 \\
\hline Nguyen Duy Thuan & & & 2 & 1980 \\
\hline Nguyen Duy Tien & 1942 & $60 ; 28$ & 36 & 1972 \\
\hline Nguyen Duy Truong & & 65 & 1 & 2019 \\
\hline Nguyen Gia Dinh & 1961 & & 2 & 1996 \\
\hline Nguyen H. Lam (HCMUT) & & $35 ; 78 ; 65$ & 3 & 2016 \\
\hline Nguyen Ha (Nguen Kha) & & & 2 & 1973 \\
\hline Nguyen Ha Thanh & 1957 & $32 ; 46$ & 4 & 1999 \\
\hline Nguyen Hac Hai & & & 4 & 1992 \\
\hline Nguyen Hai Chau & 1978 & 91 & 1 & 1999 \\
\hline Nguyen Hai Dang & 1986 & $92 ; 60 ; 34$ & 3 & 2012 \\
\hline Nguyen Hai Nam & & & 3 & 2019 \\
\hline Nguyen Hai Son & & $49 ; 35 ; 65$ & 3 & 2010 \\
\hline Nguyen Hieu Thao & & $49 ; 47 ; 90$ & 1 & 2015 \\
\hline Nguyen Ho Minh Duy & & $65 ; 35$ & 1 & 2017 \\
\hline Nguyen Hoai Minh & & $35 ; 78 ; 46$ & 62 & 2005 \\
\hline Nguyen Hoang & 1956 & $35 ; 26 ; 49$ & 11 & 1994 \\
\hline Nguyen Hoang Anh & & & 1 & 2013 \\
\hline Nguyen Hoang Giang & & & 1 & 2019 \\
\hline Nguyen Hoang Loc & & $35 ; 65 ; 78$ & 32 & 2004 \\
\hline Nguyen Hoang Luc & & $35 ; 47 ; 26$ & 18 & 2018 \\
\hline Nguyen Hoang Son & 1973 & $68 ; 05$ & 4 & 2004 \\
\hline Nguyen Hoang Thach & 1984 & & 3 & 2010 \\
\hline Nguyen Hoang Thanh & 1981 & $54 ; 57$ & 2 & 2009 \\
\hline Nguyen Hoang Tuan & & $62 ; 47 ; 35$ & 2 & 2018 \\
\hline Nguyen Hoi Nghia & 1958 & $34 ; 35 ; 46$ & 5 & 1998 \\
\hline Nguyen Hong Chuong & 1960 & & 5 & 1988 \\
\hline Nguyen Hong Duc & 1970 & $14 ; 32 ; 34$ & 6 & 2008 \\
\hline Nguyen Hong Hai & & & 1 & 2005 \\
\hline Nguyen Hong Quan & & $49 ; 54 ; 47$ & 13 & 2009 \\
\hline
\end{tabular}




\begin{tabular}{|c|c|c|c|c|}
\hline Nguyen Hong Thai & 1963 & & 1 & 1992 \\
\hline Nguyen Hung Chinh & & & 1 & 2017 \\
\hline Nguyen Hung Son & 1957 & $20 ; 16$ & 4 & 1986 \\
\hline Nguyen Huong Lam & & $68 ; 94 ; 20$ & 19 & 1990 \\
\hline Nguyen Huu Anh & 1930 & $22 ; 17 ; 20$ & 12 & 1967 \\
\hline Nguyen Huu Bao & 1950 & $60 ; 62$ & 5 & 1995 \\
\hline Nguyen Huu Bi & & & 1 & 2002 \\
\hline Nguyen Huu Can & & $35 ; 47 ; 26$ & 32 & 2018 \\
\hline Nguyen Huu Cong & 1964 & & 1 & 2018 \\
\hline Nguyen Huu Cong & 1949 & $65 ; 34$ & 43 & 1991 \\
\hline Nguyen Huu Danh & 1994 & $49 ; 90 ; 91$ & 1 & 2020 \\
\hline Nguyen Huu Dien & 1954 & 26 & 3 & 1986 \\
\hline Nguyen Huu Du & 1957 & $34 ; 60 ; 93$ & 42 & 1986 \\
\hline Nguyen Huu Duc & & & 11 & 1977 \\
\hline $\begin{array}{c}\text { Nguyen Huu Hoi (Hoi H. } \\
\text { Nguyen) }\end{array}$ & & $11 ; 60 ; 15$ & 29 & 2008 \\
\hline Nguyen Huu Khanh & 1965 & $47 ; 35$ & 3 & 2000 \\
\hline $\begin{array}{c}\text { Nguyen Huu Ngu (Nguen Hyu } \\
\text { Ngy) }\end{array}$ & & & 1 & 1966 \\
\hline Nguyen Huu Quang & 1960 & $52 ; 49 ; 58$ & 3 & 1994 \\
\hline Nguyen Huu Sau & & $93 ; 34 ; 37$ & 6 & 2014 \\
\hline Nguyen Huu Tho & 1976 & & 1 & 2002 \\
\hline Nguyen Huu Tho & 1967 & $35 ; 49$ & 2 & 2003 \\
\hline Nguyen Huu Tro & 1946 & & 2 & 1981 \\
\hline Nguyen Huu Tron & & $49 ; 90 ; 58$ & 3 & 2017 \\
\hline Nguyen Huu Tuyen & 1966 & 32 & 1 & 2003 \\
\hline Nguyen Huu Vien & & & 1 & 1989 \\
\hline Nguyen Huu Viet Hung & & $55 ; 18 ; 16$ & 36 & 1981 \\
\hline Nguyen Huy Chieu & 1979 & $49 ; 90 ; 54$ & 22 & 2008 \\
\hline Nguyen Huy Duc & & $35 ; 37 ; 76$ & 1 & 2020 \\
\hline Nguyen Huy Hoang & 1975 & $37 ; 35$ & 4 & 2000 \\
\hline
\end{tabular}




\begin{tabular}{|c|c|c|c|c|}
\hline Nguyen Huy Hung & & $13 ; 16 ; 20$ & 2 & 2011 \\
\hline Nguyen Huy Tuan & 1983 & $35 ; 47 ; 65$ & 61 & 2006 \\
\hline Nguyen Huy Tuan & & $35 ; 47 ; 26$ & 45 & 2014 \\
\hline Nguyen Huy Viet & & $54 ; 47 ; 60$ & 6 & 1982 \\
\hline Nguyen Huyen Muoi & & $34 ; 93$ & 4 & 2015 \\
\hline Nguyen Huynh Phan & 1955 & $93 ; 58 ; 55$ & 10 & 1987 \\
\hline Nguyen Khac Tin & & & 1 & 2015 \\
\hline Nguyen Khac Viet & & $14 ; 11$ & 21 & 1987 \\
\hline Nguyen Khoa Son & 1948 & $93 ; 34 ; 47$ & 66 & 1978 \\
\hline Nguyen Kieu Linh & & 65 & 1 & 2015 \\
\hline Nguyen Kieu Linh & & $51 ; 52$ & 1 & 2020 \\
\hline Nguyen Kim Khoi & & 65 & 1 & 1998 \\
\hline Nguyen Kim Ngoc & 1985 & 20 & 1 & 2016 \\
\hline Nguyen Kim Tan & & & 3 & 1993 \\
\hline Nguyen Kong Shi & & & 1 & 1982 \\
\hline Nguyen Ky Nam & & 62 & 8 & 2015 \\
\hline Nguyen Lam Tung & 1981 & 90 & 1 & 2018 \\
\hline Nguyen Le Anh & 1955 & 55 & 7 & 1981 \\
\hline Nguyen Le Hoang Anh & 1984 & $90 ; 49 ; 46$ & 9 & 2011 \\
\hline Nguyen Le Huong & & 32 & 3 & 1993 \\
\hline Nguyen Le Luc & & $47 ; 74 ; 55$ & 4 & 2003 \\
\hline Nguyen Long & & & 1 & 2012 \\
\hline Nguyen Lu Trong Khiem & & $35 ; 76$ & 3 & 2013 \\
\hline Nguyen Luong Thai Binh & & 17 & 1 & 2019 \\
\hline Nguyen Luu Son & & $93 ; 60$ & 1 & 2020 \\
\hline Nguyen Manh Cuong & 1982 & $42 ; 41$ & 2 & 2017 \\
\hline Nguyen Manh Hung & & & 2 & 1990 \\
\hline Nguyen Manh Hung & & $90 ; 46$ & 3 & 2004 \\
\hline Nguyen Manh Hung & 1956 & $35 ; 74 ; 52$ & 26 & 1988 \\
\hline Nguyen Manh Linh & & $34 ; 49 ; 93$ & 4 & 2001 \\
\hline
\end{tabular}




\begin{tabular}{|c|c|c|c|c|}
\hline Nguyen Mau Nam & 1976 & $49 ; 90 ; 58$ & 47 & 2005 \\
\hline Nguyen Minh Chuong & 1931 & $47 ; 35 ; 46$ & 71 & 1969 \\
\hline Nguyen Minh Cong & & $41 ; 46 ; 40$ & 2 & 2005 \\
\hline Nguyen Minh Dien & & $35 ; 65 ; 49$ & 5 & 2011 \\
\hline Nguyen Minh Duc & & $41 ; 54$ & 9 & 1984 \\
\hline Nguyen Minh Ha & 1955 & $51 ; 46 ; 22$ & 8 & 1994 \\
\hline Nguyen Minh Hai & & & 2 & 2013 \\
\hline Nguyen Minh Hang & & & 2 & 1997 \\
\hline Nguyen Minh Khoa & & $45 ; 42 ; 44$ & 10 & 2004 \\
\hline Nguyen Minh Man & 1953 & $34 ; 65 ; 47$ & 7 & 2003 \\
\hline Nguyen Minh Sang & 1984 & $05 ; 11$ & 2 & 2016 \\
\hline Nguyen Minh Ta & & & 1 & 1990 \\
\hline Nguyen Minh Trang & & 47 & 1 & 2019 \\
\hline Nguyen Minh Tri & & 13 & 2 & 2017 \\
\hline Nguyen Minh Tri & 1964 & $35 ; 76 ; 47$ & 50 & 1987 \\
\hline Nguyen Minh Tuan & 1969 & $45 ; 47 ; 30$ & 10 & 1992 \\
\hline Nguyen Minh Tuan & & $90 ; 49$ & 3 & 1993 \\
\hline Nguyen Minh Tung & & $90 ; 49 ; 91$ & 6 & 2015 \\
\hline Nguyen Mong & & & 2 & 1979 \\
\hline Nguyen Nam Hai & & & 1 & 1988 \\
\hline Nguyen Nam Hong & & & 5 & 1982 \\
\hline Nguyen Nang Tam & 1953 & $90 ; 49 ; 32$ & 22 & 1999 \\
\hline Nguyen Nang Thieu & & $49 ; 90$ & 1 & 2016 \\
\hline Nguyen Ngoc Chu & 1955 & & 5 & 1980 \\
\hline Nguyen Ngoc Cuong & & & 2 & 1987 \\
\hline Nguyen Ngoc Cuong & & 32 & 1 & 2020 \\
\hline Nguyen Ngoc Dai & & & 1 & 2013 \\
\hline Nguyen Ngoc Dat & & & 1 & 2012 \\
\hline Nguyen Ngoc Diep & & & 1 & 1972 \\
\hline Nguyen Ngoc Doanh & & $35 ; 78 ; 80$ & 4 & 2010 \\
\hline
\end{tabular}




\begin{tabular}{|c|c|c|c|c|}
\hline Nguyen Ngoc Hai & & $52 ; 90 ; 26$ & 16 & 1996 \\
\hline Nguyen Ngoc Hai & & $47 ; 49 ; 90$ & 1 & 2020 \\
\hline Nguyen Ngoc Hung & & 20 & 32 & 2008 \\
\hline Nguyen Ngoc Huy & & 60 & 1 & 2008 \\
\hline Nguyen Ngoc Khanh & & $58 ; 35 ; 32$ & 2 & 2015 \\
\hline Nguyen Ngoc Luan & 1984 & $90 ; 46 ; 49$ & 3 & 2018 \\
\hline Nguyen Ngoc Nhu & & $34 ; 60 ; 92$ & 1 & 2019 \\
\hline Nguyen Ngoc Phan & 1980 & & 1 & 2003 \\
\hline Nguyen Ngoc Phung & & & 1 & 2012 \\
\hline Nguyen Ngoc San & & & 1 & 1996 \\
\hline \multicolumn{2}{|c|}{ Nguyen Ngoc Thuan (Tkhuan, N. N) } & & 1 & 1998 \\
\hline Nguyen Ngoc Toi & & & 1 & 1983 \\
\hline Nguyen Ngoc Trong & & $42 ; 47 ; 35$ & 16 & 2015 \\
\hline Nguyen Ngoc Tu & & $60 ; 52$ & 1 & 2019 \\
\hline \multicolumn{2}{|c|}{ Nguyen Ngoc Tuu (Nguen Ngok Tyu) } & $90 ; 65$ & 2 & 1980 \\
\hline Nguyen Nhu Lan & & $35 ; 46 ; 65$ & 2 & 2016 \\
\hline Nguyen Nhu Ngoc & & 35 & 1 & 2020 \\
\hline Nguyen Nhu Thang & & 35 & 2 & 2008 \\
\hline Nguyen Nhut Hung & & & 8 & 2018 \\
\hline Nguyen Nhuy & 1950 & $54 ; 46 ; 47$ & 16 & 1981 \\
\hline Nguyen Nuu Du & & 49 & 1 & 2004 \\
\hline Nguyen Phuoc Tai & & $76 ; 35$ & 3 & 2019 \\
\hline Nguyen Phuong Hoa & 1996 & & 1 & 2020 \\
\hline Nguyen Phuong Mai & & 35 & 1 & 2020 \\
\hline Nguyen Phuong Thao & & 35 & 1 & 2019 \\
\hline Nguyen Quan Son & 1967 & & 1 & 2006 \\
\hline Nguyen Quang A & & & 1 & 1986 \\
\hline Nguyen Quang Dieu & 1973 & $32 ; 31 ; 46$ & 32 & 1996 \\
\hline Nguyen Quang Hoa & & 41 & 3 & 1991 \\
\hline Nguyen Quang Huy & 1973 & $90 ; 49 ; 46$ & 16 & 2002 \\
\hline
\end{tabular}




\begin{tabular}{|c|c|c|c|c|}
\hline Nguyen Quang Khanh & 1979 & & 1 & 2012 \\
\hline Nguyen Quang Minh & & & 1 & 1987 \\
\hline Nguyen Quang Thai & & & 6 & 1967 \\
\hline Nguyen Quang Thang & & 35 & 1 & 2011 \\
\hline \multicolumn{2}{|c|}{ Nguyen Quoc Dan (Nguen Kuok Zan) } & & 2 & 1968 \\
\hline Nguyen Quoc Hung & & 35 & 1 & 2004 \\
\hline Nguyen Quoc Hung (Shanghai) & 1988 & $35 ; 31 ; 76$ & 24 & 2014 \\
\hline Nguyen Quoc Son & 1937 & & 1 & 2004 \\
\hline Nguyen Quoc Thang & 1957 & $20 ; 11 ; 14$ & 53 & 1989 \\
\hline Nguyen Quoc Thi & & & 2 & 1971 \\
\hline Nguyen Quoc Tho & 1973 & $19 ; 46 ; 55$ & 2 & 1999 \\
\hline Nguyen Quoc Toan & & & 2 & 1978 \\
\hline Nguyen Quoc Tuan & & $34 ; 93$ & 1 & 2009 \\
\hline Nguyen Quoc Tuan & & $49 ; 90$ & 1 & 2020 \\
\hline Nguyen Quy & & & 1 & 1988 \\
\hline Nguyen Quy Hy & 1939 & 60 & 10 & 1974 \\
\hline Nguyen Quy Khang & & $68 ; 20 ; 94$ & 4 & 1985 \\
\hline Nguyen Quyen & & $76 ; 35 ; 82$ & 1 & 2020 \\
\hline Nguyen Quynh Hoa & 1989 & $91 ; 49 ; 90$ & 3 & 2016 \\
\hline Nguyen Quynh Lan & & & 2 & 1989 \\
\hline Nguyen Quynh Nga & 1976 & $47 ; 54$ & 5 & 2001 \\
\hline Nguyen S. N & & & 1 & 1986 \\
\hline Nguyen Si Hoang & 1978 & 65 & 2 & 2006 \\
\hline $\begin{array}{l}\text { Nguyen Si Hoang (Nguen Si } \\
\text { Hong) }\end{array}$ & & & 1 & 1968 \\
\hline Nguyen Si Minh & & & 4 & 1977 \\
\hline Nguyen Sinh Bay & 1951 & $34 ; 15 ; 39$ & 6 & 1999 \\
\hline Nguyen Song Ha & & $47 ; 49$ & 2 & 2016 \\
\hline Nguyen Sum & 1961 & 55 & 14 & 1992 \\
\hline Nguyen Sy Anh Tuan & 1957 & & 2 & 1995 \\
\hline Nguyen Sy Thai Ha & & & 1 & 2012 \\
\hline
\end{tabular}




\begin{tabular}{|c|c|c|c|c|}
\hline $\begin{array}{c}\text { Nguyen Sy Tuan (Nguen Si } \\
\text { Tuen) }\end{array}$ & & & 1 & 1971 \\
\hline Nguyen T. T. & & & 3 & 1991 \\
\hline Nguyen T. Trinh & & 35 & 3 & 2018 \\
\hline Nguyen Tam & & & 1 & 1968 \\
\hline \multicolumn{2}{|c|}{ Nguyen Tam Nhan (Nhan-Tam Nguyen) } & & 2 & 2014 \\
\hline Nguyen Tan Hoa & 1968 & $45 ; 47$ & 4 & 2000 \\
\hline Nguyen Tat Dat & 1999 & & 1 & 2020 \\
\hline Nguyen Tat Thang & 1982 & $14 ; 32 ; 57$ & 9 & 2011 \\
\hline Nguyen Thac Dung & 1980 & $32 ; 58 ; 53$ & 5 & 2005 \\
\hline Nguyen Thai An & 1984 & $49 ; 90 ; 58$ & 11 & 2012 \\
\hline Nguyen Thai Hoa & 1978 & $13 ; 16$ & 4 & 1999 \\
\hline Nguyen Thai Son & 1959 & $32 ; 58$ & 4 & 1998 \\
\hline Nguyen Thanh Anh & & 35 & 1 & 2016 \\
\hline Nguyen Thanh Bang & & & 2 & 1974 \\
\hline Nguyen Thanh Binh & & $60 ; 91$ & 3 & 2001 \\
\hline Nguyen Thanh Chung & & $35 ; 34 ; 47$ & 3 & 2020 \\
\hline Nguyen Thanh Dieu & & $60 ; 34 ; 92$ & 12 & 2013 \\
\hline Nguyen Thanh Hai & & $44 ; 33$ & 3 & 1991 \\
\hline Nguyen Thanh Hao & 1982 & $47 ; 49$ & 1 & 2006 \\
\hline Nguyen Thanh Hong & 1980 & $44 ; 33 ; 45$ & 6 & 2007 \\
\hline Nguyen Thanh Hung & & 90 & 3 & 2019 \\
\hline Nguyen Thanh Huong & & & 1 & 1988 \\
\hline Nguyen Thanh Long & & & 1 & 1993 \\
\hline Nguyen Thanh Long & 1972 & $90 ; 93$ & 1 & 2004 \\
\hline Nguyen Thanh Long & 1956 & $35 ; 65 ; 45$ & 79 & 1986 \\
\hline Nguyen Thanh Nhan & & $35 ; 65 ; 76$ & 16 & 2013 \\
\hline Nguyen Thanh Quang & 1957 & $11 ; 32 ; 30$ & 9 & 1998 \\
\hline Nguyen Thanh Qui & & 49 & 5 & 2010 \\
\hline Nguyen Thanh Son & 1976 & 11 & 1 & 2018 \\
\hline Nguyen Thanh Thien & & 37 & 1 & 2017 \\
\hline
\end{tabular}




\begin{tabular}{|c|c|c|c|c|}
\hline Nguyen Thanh Thuy & & & 2 & 1985 \\
\hline Nguyen Thanh Tuan & & 14 & 1 & 2006 \\
\hline Nguyen Thanh Tung & 1986 & $47 ; 42 ; 35$ & 3 & 2016 \\
\hline Nguyen Thanh Tung & & $47 ; 34 ; 35$ & 1 & 2020 \\
\hline $\begin{array}{l}\text { Nguyen Thanh Tung } \\
\text { (Nanyang) }\end{array}$ & 1993 & $68 ; 93$ & 1 & 2015 \\
\hline Nguyen Thanh Van & & $31 ; 35 ; 30$ & 9 & 2000 \\
\hline Nguyen Thanh Van & 1943 & $30 ; 31 ; 35$ & 5 & 1997 \\
\hline Nguyen Thanh $\mathrm{Vu}$ & 1960 & $58 ; 35$ & 2 & 2005 \\
\hline Nguyen The Cuong & & $18 ; 55$ & $\overline{4}$ & 2015 \\
\hline Nguyen The Hoan & 1941 & $34 ; 41 ; 65$ & 11 & 1968 \\
\hline Nguyen The Hung & & & 1 & 2015 \\
\hline Nguyen The Long & & & 2 & 1975 \\
\hline Nguyen The Vinh & 1980 & $47 ; 65 ; 68$ & 10 & 2005 \\
\hline Nguyen Thi Bach Kim & 1961 & 90 & 9 & 2000 \\
\hline Nguyen Thi Dung & 1964 & 13 & 5 & 2004 \\
\hline Nguyen Thi Hien & & $34 ; 93 ; 94$ & 3 & 2013 \\
\hline Nguyen Thi Hoa & & 60 & 1 & 1995 \\
\hline Nguyen Thi Hoai Phuong & & $90 ; 78 ; 65$ & $\overline{6}$ & 2002 \\
\hline Nguyen Thi Hong & & & 3 & 1968 \\
\hline Nguyen Thi Hong & & $42 ; 47$ & 2 & 2016 \\
\hline Nguyen Thi Hong & & $34 ; 47 ; 90$ & 3 & 2015 \\
\hline Nguyen Thi Hong Loan & & 13 & 5 & 1999 \\
\hline Nguyen Thi Hong Minh & 1968 & $65 ; 34$ & 3 & 2000 \\
\hline Nguyen Thi Hong Nhung & & $35 ; 46$ & 1 & 2020 \\
\hline Nguyen Thi Kieu & 1986 & & 2 & 2017 \\
\hline Nguyen Thi Kieu Nga & 1975 & 13 & 1 & 2010 \\
\hline $\begin{array}{c}\text { Nguyen Thi Kim Anh (N. K. } \\
\text { Anh) }\end{array}$ & 1963 & & 1 & 1991 \\
\hline Nguyen Thi Kim Chuc & & & 4 & 2009 \\
\hline Nguyen Thi Kim Son & & $03 ; 35 ; 46$ & 2 & 2008 \\
\hline
\end{tabular}




\begin{tabular}{|c|c|c|c|c|}
\hline Nguyen Thi Lan Huong & 1979 & $34 ; 32$ & 2 & 2019 \\
\hline Nguyen Thi Le & & 54 & 1 & 2008 \\
\hline Nguyen Thi Le Trang & & 90 & 1 & 2007 \\
\hline Nguyen Thi Loan & & $35 ; 34$ & 1 & 2020 \\
\hline Nguyen Thi Minh & & 60 & 1 & 1992 \\
\hline Nguyen Thi Minh Hue & & 90 & 1 & 2006 \\
\hline Nguyen Thi Minh Khai & & 42 & 1 & 2015 \\
\hline Nguyen Thi Minh Toai & & $35 ; 76$ & 1 & 2020 \\
\hline Nguyen Thi Nga & & $35 ; 53$ & 3 & 2004 \\
\hline Nguyen Thi Ngan & & $45 ; 46 ; 42$ & 2 & 2011 \\
\hline Nguyen Thi Ngan & & $76 ; 35 ; 37$ & 2 & 2020 \\
\hline Nguyen Thi Ngan & & $45 ; 47 ; 42$ & 1 & 2010 \\
\hline Nguyen Thi Ngoc Anh & & & 1 & 2010 \\
\hline Nguyen Thi Ngoc Diep & 1984 & $14 ; 11 ; 12$ & 2 & 2012 \\
\hline Nguyen Thi Ngoc Oanh & 1985 & 65 & 4 & 2016 \\
\hline Nguyen Thi Nhung & & $30 ; 32$ & 3 & 2017 \\
\hline Nguyen Thi Phong & & $35 ; 45 ; 78$ & 1 & 2015 \\
\hline Nguyen Thi Phuong Dung & 1972 & $17 ; 16 ; 20$ & 4 & 2003 \\
\hline Nguyen Thi Quynh Anh & 1981 & $91 ; 49 ; 90$ & 2 & 2011 \\
\hline Nguyen Thi Quynh Phuong & & & 1 & 2017 \\
\hline Nguyen Thi Quynh Trang & & $49 ; 90$ & 2 & 2012 \\
\hline Nguyen Thi Tam Bach & & & 3 & 1969 \\
\hline Nguyen Thi Thanh Ha & & $90 ; 65 ; 47$ & 2 & 2020 \\
\hline Nguyen Thi Thanh Ha & & 47 & 2 & 2008 \\
\hline Nguyen Thi Thanh Hien & & $32 ; 30$ & 1 & 2018 \\
\hline Nguyen Thi Thanh Huyen & & $34 ; 93$ & 3 & 2016 \\
\hline Nguyen Thi Thanh Huyen & & 68 & 1 & 2006 \\
\hline Nguyen Thi Thanh Tam & 1993 & 13 & 2 & 2019 \\
\hline Nguyen Thi Thao & 1979 & $14 ; 11 ; 26$ & 6 & 2009 \\
\hline Nguyen Thi Thao Truc & & 35 & 2 & 2005 \\
\hline
\end{tabular}




\begin{tabular}{|c|c|c|c|c|}
\hline Nguyen Thi The & & $34 ; 60 ; 93$ & 3 & 2010 \\
\hline Nguyen Thi Thu Hang & & $32 ; 30$ & 1 & 2017 \\
\hline Nguyen Thi Thu Hien & & 60 & 1 & 2020 \\
\hline Nguyen Thi Thu Huong & 1975 & $90 ; 49$ & 7 & 2011 \\
\hline Nguyen Thi Thu Thuy & & $47 ; 41 ; 49$ & 9 & 2008 \\
\hline Nguyen Thi Thu Van & & $90 ; 26$ & 2 & 2003 \\
\hline Nguyen Thi Thuy & & 60 & 1 & 2014 \\
\hline Nguyen Thi Thuy Loan & & & 1 & 2013 \\
\hline Nguyen Thi Thuy Quynh & & $93 ; 60 ; 34$ & 3 & 2010 \\
\hline Nguyen Thi Tinh & & & 3 & 1995 \\
\hline Nguyen Thi Toan & 1982 & $49 ; 90 ; 93$ & 10 & 2010 \\
\hline Nguyen Thi Tuyet Mai & & $32 ; 46$ & 5 & 2000 \\
\hline Nguyen Thi Van Anh & & 13 & 1 & 2018 \\
\hline Nguyen Thi Van Hang & 1987 & $90 ; 49$ & 4 & 2014 \\
\hline Nguyen Thi Vinh & & & 1 & 2016 \\
\hline Nguyen Thi Yen Ngoc & & $65 ; 35$ & 1 & 2015 \\
\hline Nguyen Thi Yen Nhi & & $49 ; 90$ & 1 & 2014 \\
\hline Nguyen Thien Luan & & & 2 & 1985 \\
\hline Nguyen Thiep & & & 1 & 1979 \\
\hline Nguyen Thieu Huy & 1975 & $34 ; 35 ; 47$ & 21 & 2001 \\
\hline Nguyen Thinh & 1980 & 47 & 4 & 2004 \\
\hline Nguyen Thu Ha & 1983 & $34 ; 06 ; 65$ & 1 & 2016 \\
\hline Nguyen Thu Hang & 1982 & 13 & 3 & 2017 \\
\hline Nguyen Thu Huong & 1979 & 90 & 1 & 2019 \\
\hline Nguyen Thu Nga & & 46 & 5 & 1991 \\
\hline Nguyen Thu Thuy & & $65 ; 60$ & 1 & 2020 \\
\hline Nguyen Thu Thuy & & 65 & 1 & 2012 \\
\hline Nguyen Thua Hop & 1932 & 35 & 17 & 1964 \\
\hline Nguyen Thuan Bang & & & 1 & 1993 \\
\hline Nguyen Thuc Loan & & & 9 & 1970 \\
\hline
\end{tabular}




\begin{tabular}{|c|c|c|c|c|}
\hline Nguyen Thuong Uan & & & 6 & 1975 \\
\hline Nguyen Thuy Anh & 1974 & & 1 & 1996 \\
\hline Nguyen Thuy Thanh & 1941 & $41 ; 30$ & 2 & 1983 \\
\hline Nguyen Tien Da & 1987 & & 2 & 2017 \\
\hline Nguyen Tien Dai & 1952 & 35 & 5 & 1980 \\
\hline Nguyen Tien Dung & & $37 ; 53 ; 58$ & 15 & 1997 \\
\hline Nguyen Tien Dung & & $60 ; 90 ; 93$ & 2 & 2008 \\
\hline Nguyen Tien Khai & 1984 & $49 ; 58 ; 35$ & 29 & 2007 \\
\hline Nguyen Tien Khiem & 1955 & & 11 & 1983 \\
\hline Nguyen Tien Manh & 1964 & & 1 & 2006 \\
\hline Nguyen Tien Quang & & 18 & 6 & 1987 \\
\hline Nguyen Tien Tai & & 30 & 3 & 1981 \\
\hline Nguyen Tien Trung & & $46 ; 32 ; 35$ & 2 & 2004 \\
\hline Nguyen Tien Yet & 1985 & $34 ; 37$ & 2 & 2011 \\
\hline Nguyen To Nhu & 1953 & $54 ; 46 ; 58$ & 40 & 1979 \\
\hline Nguyen Toan Thang & & & 1 & 1989 \\
\hline Nguyen Tran Thuan & & $60 ; 65 ; 52$ & 2 & 2014 \\
\hline Nguyen Trong Hieu & 1982 & & 1 & 2006 \\
\hline Nguyen Trong Hoa & & $32 ; 30$ & 2 & 2006 \\
\hline Nguyen Trong Toan & & & 2 & 1982 \\
\hline $\begin{array}{c}\text { Nguyen Trong Toan (Nguyen } \\
\text { T. Toan) }\end{array}$ & 1979 & $35 ; 76 ; 82$ & 43 & 2003 \\
\hline Nguyen Trung Hieu & & 47 & 1 & 2020 \\
\hline Nguyen Trung Hung & 1945 & 62 & 2 & 1998 \\
\hline Nguyen Trung Kien & 1986 & 90 & 5 & 2019 \\
\hline Nguyen Trung Thanh & 1980 & $35 ; 65 ; 80$ & 11 & 2007 \\
\hline Nguyen Trung Thanh & & 68 & 12 & 2012 \\
\hline Nguyen Truong Thanh & 1980 & & 13 & 2005 \\
\hline Nguyen Tu Cuong & 1951 & $13 ; 14 ; 16$ & 58 & 1977 \\
\hline Nguyen Tu Thanh & & & 4 & 1972 \\
\hline Nguyen Tuan Duy & & $35 ; 26 ; 46$ & 3 & 2013 \\
\hline
\end{tabular}




\begin{tabular}{|c|c|c|c|c|}
\hline Nguyen Tuan Long & 1981 & 13 & 2 & 2015 \\
\hline Nguyen Tuong & 1938 & $35 ; 65$ & 5 & 1978 \\
\hline Nguyen V Chan & & & 1 & 1991 \\
\hline Nguyen V Kh & & & 2 & 1986 \\
\hline Nguyen Van An & & $32 ; 30$ & 2 & 2017 \\
\hline Nguyen Van Bao & & & 1 & 1980 \\
\hline Nguyen Van Chau & 1955 & $14 ; 32 ; 34$ & 22 & 1985 \\
\hline Nguyen Van Co & 1950 & $11 ; 47 ; 54$ & 5 & 1999 \\
\hline Nguyen Van Dac & & & 1 & 2017 \\
\hline Nguyen Van Dao & 1937 & 34 & 28 & 1968 \\
\hline Nguyen Van Dinh & & 34 & 4 & 2000 \\
\hline Nguyen Van Dong & 1955 & 32 & 6 & 1986 \\
\hline Nguyen Van Duc & 1981 & $35 ; 47 ; 65$ & 11 & 2008 \\
\hline Nguyen Van Duc & & & 1 & 2012 \\
\hline Nguyen Van Dung & 1981 & $54 ; 47 ; 39$ & 7 & 2006 \\
\hline Nguyen Van Gia & 1930 & & 2 & 1976 \\
\hline Nguyen Van Giang & & & 4 & 1988 \\
\hline Nguyen Van Hanh & 1980 & 53 & 1 & 2002 \\
\hline Nguyen Van Hao & 1970 & 46 & 4 & 1998 \\
\hline Nguyen Van Hien & & $90 ; 49 ; 47$ & 11 & 2004 \\
\hline Nguyen Van Hieu & 1938 & $34 ; 81$ & 33 & 1963 \\
\hline Nguyen Van Hieu & & & 2 & 1994 \\
\hline Nguyen Van Ho & 1947 & $60 ; 62$ & 12 & 1976 \\
\hline Nguyen Van Hoang & & & 2 & 1993 \\
\hline Nguyen Van Hoang & 1976 & $13 ; 18$ & 7 & 2005 \\
\hline Nguyen Van Hoang & & $46 ; 26 ; 35$ & 1 & 2020 \\
\hline Nguyen Van Hoang (1985) & 1985 & $26 ; 46 ; 52$ & 26 & 2011 \\
\hline Nguyen Van Hong & & & 1 & 2020 \\
\hline Nguyen Van Huan & & 60 & 2 & 2014 \\
\hline Nguyen Van Hung & & & 8 & 1987 \\
\hline
\end{tabular}




\begin{tabular}{|c|c|c|c|c|}
\hline Nguyen Van Hung & & $47 ; 49 ; 58$ & 3 & 2020 \\
\hline Nguyen Van Huu & 1941 & $62 ; 60$ & 8 & 1972 \\
\hline Nguyen Van Khai & & & 3 & 1992 \\
\hline Nguyen Van Khiem & 1977 & 41 & 4 & 2005 \\
\hline Nguyen Van Khue & 1944 & $32 ; 46 ; 30$ & 58 & 1965 \\
\hline Nguyen Van Kinh & & $47 ; 65$ & 3 & 1989 \\
\hline Nguyen Van Long & 1963 & 68 & 1 & 2007 \\
\hline Nguyen Van Luong & & $55 ; 49 ; 54$ & 2 & 2014 \\
\hline Nguyen Van Man & & & 1 & 1991 \\
\hline Nguyen Van Manh & 1954 & & 1 & 1990 \\
\hline Nguyen Van Mau & 1951 & $45 ; 47 ; 34$ & 39 & 1982 \\
\hline Nguyen Van Minh & & $65 ; 34$ & 2 & 2007 \\
\hline Nguyen Van Minh & 1966 & $34 ; 47 ; 35$ & 114 & 1987 \\
\hline Nguyen Van Minh & & & 1 & 2017 \\
\hline Nguyen Van Minh (FTU) & & $58 ; 37 ; 53$ & 2 & 2013 \\
\hline Nguyen Van Nghi & & & 1 & 2000 \\
\hline Nguyen Van Ngoc & 1949 & $42 ; 46 ; 47$ & 18 & 1979 \\
\hline Nguyen Van Nhan & & $35 ; 30 ; 41$ & 6 & 1998 \\
\hline Nguyen Van Ninh & & $14 ; 52 ; 55$ & 1 & 2017 \\
\hline Nguyen Van Phu & & 32 & 4 & 2012 \\
\hline Nguyen Van Quang & 1957 & $60 ; 46 ; 47$ & 18 & 1992 \\
\hline Nguyen Van Quang & & $35 ; 90$ & 3 & 2010 \\
\hline Nguyen Van Quy & & $90 ; 47 ; 65$ & 9 & 2001 \\
\hline Nguyen Van Sanh & & 16 & 16 & 1993 \\
\hline Nguyen Van Son & 1977 & & 1 & 2003 \\
\hline Nguyen Van Son & & & 1 & 2016 \\
\hline Nguyen Van Su & & & 2 & 1984 \\
\hline Nguyen Van Thang & 1987 & $35 ; 47 ; 65$ & 4 & 2015 \\
\hline Nguyen Van Thanh & & $35 ; 60 ; 37$ & 5 & 2012 \\
\hline Nguyen Van The & 1998 & & 1 & 2020 \\
\hline
\end{tabular}




\begin{tabular}{|c|c|c|c|c|}
\hline Nguyen Van Thin & & $30 ; 35 ; 32$ & 21 & 2014 \\
\hline Nguyen Van Thinh & & $35 ; 47 ; 65$ & 9 & 2013 \\
\hline Nguyen Van Thoai & 1950 & $90 ; 65$ & 29 & 1978 \\
\hline Nguyen Van Thu & 1946 & $60 ; 19 ; 33$ & 35 & 1976 \\
\hline Nguyen Van Thuong & & & 3 & 1984 \\
\hline Nguyen Van Tin & & & 1 & 1975 \\
\hline Nguyen Van Toan & & $62 ; 60$ & 7 & 1998 \\
\hline Nguyen Van Trao & 1973 & 32 & 9 & 2000 \\
\hline Nguyen Van Truyen & & $35 ; 22 ; 26$ & 4 & 2005 \\
\hline Nguyen Van Tuan & & & 5 & 1995 \\
\hline Nguyen Van Tuyen & & $90 ; 49 ; 58$ & 9 & 2012 \\
\hline Nguyen Van Ty & & & 2 & 1977 \\
\hline Nguyen Van Vu & & $49 ; 90$ & 1 & 2020 \\
\hline Nguyen Van Vy & 1944 & & 5 & 1977 \\
\hline Nguyen Van Y & & $35 ; 74$ & 2 & 2016 \\
\hline Nguyen Viet Anh & 1974 & $32 ; 37 ; 60$ & 9 & 2010 \\
\hline Nguyen Viet Duc & 1959 & & 1 & 1994 \\
\hline Nguyen Viet Dung & & $14 ; 16 ; 32$ & 20 & 1983 \\
\hline Nguyen Viet Dung & & $16 ; 18$ & 23 & 1988 \\
\hline Nguyen Viet Hai & & $57 ; 22$ & 3 & 2001 \\
\hline Nguyen Viet Hung & 1973 & 28 & 2 & 2006 \\
\hline Nguyen Viet Linh & & $35 ; 53$ & 18 & 2003 \\
\hline Nguyen Viet Phu & & & 2 & 1974 \\
\hline Nguyen Viet Phuong & & $30 ; 35$ & 2 & 2017 \\
\hline Nguyen Viet Trieu Tien & 1950 & & 1 & 1985 \\
\hline Nguyen Viet Tuan & & 35 & 2 & 2017 \\
\hline Nguyen Vu Duy Linh & & 26 & 2 & 2001 \\
\hline Nguyen Vu Huy & & $44 ; 65 ; 47$ & 3 & 2002 \\
\hline Nguyen Vu Luong & 1954 & $45 ; 47 ; 39$ & 2 & 1996 \\
\hline Nguyen Vu Tien & 1951 & 49 & 2 & 1997 \\
\hline
\end{tabular}




\begin{tabular}{|c|c|c|c|c|}
\hline Nguyen Xuan Ha & & $26 ; 90 ; 49$ & 3 & 2000 \\
\hline Nguyen Xuan Hai & & $49 ; 47 ; 91$ & 7 & 2006 \\
\hline Nguyen Xuan Hoai & & & 2 & 2017 \\
\hline Nguyen Xuan Hong & 1983 & 32 & 16 & 2010 \\
\hline Nguyen Xuan Hung & & 90 & 1 & 2001 \\
\hline Nguyen Xuan Hung & & $65 ; 74$ & 1 & 2015 \\
\hline Nguyen Xuan Huy & 1957 & & 12 & 1985 \\
\hline Nguyen Xuan Ky & & $41 ; 42$ & 26 & 1972 \\
\hline Nguyen Xuan Lai & & $11 ; 30$ & 1 & 2012 \\
\hline Nguyen Xuan Loc & 1935 & $60 ; 31$ & 21 & 1967 \\
\hline Nguyen Xuan My & 1940 & & 16 & 1980 \\
\hline Nguyen Xuan Nguyen & & & 6 & 1968 \\
\hline Nguyen Xuan Tan & 1950 & $49 ; 90 ; 47$ & 63 & 1980 \\
\hline Nguyen Xuan Thanh & 1971 & & 2 & 2009 \\
\hline Nguyen Xuan Thanh & & $35 ; 76 ; 65$ & 2 & 2020 \\
\hline Nguyen Xuan Thao & 1957 & $44 ; 33 ; 45$ & 28 & 1994 \\
\hline Nguyen Xuan Thuan & & $60 ; 47$ & 6 & 2001 \\
\hline Nguyen Xuan Tu & & 35 & 1 & 2017 \\
\hline Nguyen Xuan Tuyen & 1949 & $16 ; 18 ; 20$ & 17 & 1976 \\
\hline Ninh Quang Hai & 1952 & 70 & 2 & 1999 \\
\hline Ninh Van Thu & 1980 & $32 ; 37 ; 51$ & 11 & 2009 \\
\hline Nong Quoc Chinh & & & 1 & 2005 \\
\hline Oanh Nguyen & 1989 & $60 ; 05 ; 68$ & 7 & 2016 \\
\hline Ong Thanh Hai & & $65 ; 74 ; 35$ & 3 & 2015 \\
\hline P. H. Thoa & & & 1 & 1991 \\
\hline P. T. Thuy & & 35 & 3 & 2009 \\
\hline Pham An Vinh & 1983 & $13 ; 14$ & 1 & 2016 \\
\hline Pham Anh Minh & 1960 & $20 ; 55 ; 17$ & 33 & 1982 \\
\hline Pham Canh Duong & 1949 & $90 ; 65$ & 7 & 1978 \\
\hline Pham Chi Vinh & 1959 & & 2 & 2017 \\
\hline
\end{tabular}




\begin{tabular}{|c|c|c|c|c|}
\hline Pham D. Dat & & $49 ; 90$ & 2 & 2018 \\
\hline Pham Dinh Huong & & $54 ; 32$ & 1 & 2003 \\
\hline Pham Dinh Tao & 1955 & $90 ; 65 ; 49$ & 14 & 1995 \\
\hline Pham Dinh Tung & & 62 & 6 & 2015 \\
\hline Pham Du & 1976 & 65 & 1 & 2006 \\
\hline Pham Duc Chinh & 1958 & 74 & 23 & 1991 \\
\hline Pham Duc Hiep & 1984 & 35 & 2 & 2017 \\
\hline Pham Duc Thoan & & $32 ; 14 ; 30$ & 15 & 2011 \\
\hline Pham Duy Dat & & & 1 & 2012 \\
\hline Pham Duy Khanh & & $49 ; 47 ; 26$ & 9 & 2013 \\
\hline Pham Gia Hung & 1963 & $49 ; 90$ & 3 & 2011 \\
\hline Pham Gia Thu & 1944 & 62 & 3 & 2001 \\
\hline Pham Hien Bang & 1955 & $46 ; 32 ; 31$ & 6 & 1998 \\
\hline Pham Hoang Giang & 1995 & & 2 & 2017 \\
\hline Pham Hoang $\mathrm{Ha}$ & 1981 & $30 ; 32 ; 53$ & 7 & 2010 \\
\hline Pham Hoang Hiep & 1982 & $32 ; 31 ; 14$ & 39 & 2005 \\
\hline Pham Hoang Quan & 1966 & $35 ; 65 ; 44$ & 13 & 2004 \\
\hline Pham Hong Nam & & 13 & 2 & 2015 \\
\hline Pham Hong Quang & 1966 & & 10 & 1982 \\
\hline Pham Hung Quy & 1983 & $13 ; 14$ & 26 & 2010 \\
\hline Pham Huu Anh Ngoc & 1967 & $34 ; 93 ; 39$ & 35 & 1998 \\
\hline Pham Huu Khanh & 1975 & 13 & 2 & 2010 \\
\hline Pham Huu Sach & 1941 & $49 ; 90 ; 26$ & 80 & 1968 \\
\hline Pham Huu Tiep & & $20 ; 11 ; 17$ & 160 & 1986 \\
\hline Pham Huu Tri & & 35 & 1 & 1995 \\
\hline Pham Huy Dien & 1949 & $90 ; 49$ & 18 & 1981 \\
\hline Pham Huy Tung & 1977 & 26 & 1 & 2007 \\
\hline Pham Khac Ban & & $58 ; 32$ & 5 & 1991 \\
\hline Pham Kim Quy & & $65 ; 47 ; 91$ & 3 & 2020 \\
\hline Pham Ky Anh & 1949 & $47 ; 65 ; 34$ & 46 & 1974 \\
\hline
\end{tabular}




\begin{tabular}{|c|c|c|c|c|}
\hline Pham Loi Vu & 1934 & $35 ; 37 ; 34$ & 29 & 1971 \\
\hline Pham Minh Hien & 1958 & $65 ; 35 ; 34$ & 4 & 2002 \\
\hline Pham Minh Thong & & $39 ; 34 ; 92$ & 1 & 2011 \\
\hline Pham Ngoc Anh & 1956 & $16 ; 13 ; 06$ & 50 & 1978 \\
\hline Pham Ngoc Anh & 1970 & $90 ; 65 ; 49$ & 21 & 2004 \\
\hline Pham Ngoc Anh Cuong & 1962 & $57 ; 55 ; 19$ & 3 & 1985 \\
\hline Pham Ngoc Boi & 1959 & $34 ; 41 ; 47$ & 4 & 1999 \\
\hline $\begin{array}{c}\text { Pham Ngoc Can (Fam Ngok } \\
\text { Can) }\end{array}$ & & & 1 & 1968 \\
\hline Pham Ngoc Khoi & & & 2 & 1986 \\
\hline Pham Ngoc Mai & & $32 ; 54$ & 3 & 2003 \\
\hline Pham Ngoc Thao & 1946 & & 2 & 1988 \\
\hline Pham Nguyen Thu Trang & 1980 & $32 ; 54 ; 46$ & 6 & 2003 \\
\hline Pham Phu Phat & & $14 ; 32 ; 58$ & 2 & 2018 \\
\hline Pham Quang Dung & & & 3 & 2012 \\
\hline Pham Quang Hung & 1965 & & 2 & 1995 \\
\hline Pham Quang Trinh & & $46 ; 54 ; 18$ & 4 & 1997 \\
\hline Pham Quy Muoi & 1980 & $35 ; 65 ; 49$ & 5 & 2013 \\
\hline Pham Thanh Duoc & & $90 ; 49 ; 91$ & 1 & 2020 \\
\hline Pham Thanh Duong & & $65 ; 41 ; 35$ & 3 & 2020 \\
\hline Pham Thanh Hieu & & $47 ; 41$ & 2 & 2016 \\
\hline Pham Thanh Huyen & & & 1 & 2019 \\
\hline Pham Thanh Son & & 35 & 1 & 2011 \\
\hline Pham Thanh Tri & 1950 & & 1 & 1986 \\
\hline Pham The Anh & 1985 & $37 ; 60 ; 47$ & 6 & 2014 \\
\hline Pham The Lai & & & 1 & 1978 \\
\hline Pham The Long & 1954 & $90 ; 65$ & 12 & 1981 \\
\hline Pham The Que & & & 2 & 1987 \\
\hline Pham Thi Bach Ngoc & 1974 & $47 ; 34 ; 15$ & 4 & 2001 \\
\hline Pham Thi Hoai & & $47 ; 49 ; 90$ & 1 & 2020 \\
\hline Pham Thi Lan & & & 2 & 2009 \\
\hline
\end{tabular}




\begin{tabular}{|c|c|c|c|c|}
\hline Pham Thi Minh Tam & & $35 ; 47$ & 2 & 2017 \\
\hline Pham Thi Thu Hoai & & $47 ; 49$ & 1 & 2020 \\
\hline Pham Thi Thuy & & 35 & 1 & 2019 \\
\hline Pham Thi Trang & 1987 & $37 ; 35$ & 5 & 2013 \\
\hline Pham Thi Vui & & $54 ; 49 ; 80$ & 1 & 2020 \\
\hline Pham Tien Son & 1964 & $32 ; 14 ; 90$ & 47 & 1997 \\
\hline Pham Tra An & 1944 & 68 & 9 & 1976 \\
\hline Pham Tran Nhu & 1945 & & 19 & 1979 \\
\hline Pham Tri Nguyen & 1980 & $60 ; 28$ & 1 & 2015 \\
\hline Pham Trieu Duong & 1971 & 35 & 3 & 2004 \\
\hline Pham Trong Quat & 1950 & & 3 & 1984 \\
\hline Pham Trong Tien & 1987 & $47 ; 32 ; 30$ & 9 & 2017 \\
\hline Pham Trung Kien & & 90 & 3 & 2000 \\
\hline Pham Truong Hoang Nhan & 1996 & & 1 & 2020 \\
\hline Pham Tuan A & & & 1 & 2019 \\
\hline Pham Tuan Anh & & & 2 & 2017 \\
\hline Pham Van Bang & & $34 ; 35$ & 1 & 2017 \\
\hline Pham Van Binh & & & 1 & 2012 \\
\hline Pham Van Chien & & & 1 & 2019 \\
\hline Pham Van Chung & & 11 & 5 & 1993 \\
\hline Pham Van Chung & 1960 & $62 ; 60$ & 1 & 2007 \\
\hline Pham Van Hai & & & 2 & 2019 \\
\hline Pham Van Hien & & $47 ; 34 ; 35$ & 1 & 2020 \\
\hline Pham Van Hoang & & $76 ; 42 ; 47$ & 1 & 2016 \\
\hline Pham Van Huy & & $90 ; 65 ; 49$ & 3 & 2020 \\
\hline Pham Van Loi & & $49 ; 58 ; 65$ & 1 & 2004 \\
\hline Pham Van Luong & 1988 & & 1 & 2012 \\
\hline Pham Van Minh & & 16 & 1 & 2004 \\
\hline Pham Van Quoc & & 49 & 1 & 2004 \\
\hline Pham Van Son & & $90 ; 49 ; 47$ & 2 & 2007 \\
\hline
\end{tabular}




\begin{tabular}{|c|c|c|c|c|}
\hline Pham Van Thang & 1990 & $11 ; 05 ; 52$ & 29 & 2012 \\
\hline Pham Van Thao & & 68 & 2 & 1999 \\
\hline Pham Van Trung & 1985 & $05 ; 68 ; 90$ & 12 & 2011 \\
\hline Pham Van Viet & & $35 ; 34$ & 5 & 2004 \\
\hline Pham Viet Duc & & $32 ; 30$ & 9 & 1999 \\
\hline Pham Viet Hai & 1986 & $47 ; 34 ; 30$ & 19 & 2010 \\
\hline Pham Viet Hung & 1986 & $60 ; 26 ; 30$ & 9 & 2013 \\
\hline Pham Viet Hung & 1955 & $16 ; 15 ; 20$ & 10 & 1986 \\
\hline Pham Vu Long & & & 1 & 2012 \\
\hline Pham Xuan Binh & & $60 ; 62$ & 3 & 1998 \\
\hline Pham Xuan Du & 1976 & $65 ; 35 ; 34$ & 4 & 2002 \\
\hline Pham Xuan Huyen & 1980 & $60 ; 93 ; 91$ & 77 & 1996 \\
\hline Pham Xuan Trung & & 90 & 1 & 2006 \\
\hline Phan Dan & & 16 & 9 & 1988 \\
\hline Phan Dang Cau & & & 5 & 1980 \\
\hline Phan Dinh Dieu & 1936 & & 28 & 1965 \\
\hline Phan Dinh Phung & & $34 ; 26 ; 47$ & 8 & 2011 \\
\hline Phan Duc Chau & & & 3 & 1981 \\
\hline Phan Duc Chinh & 1936 & & 2 & 1965 \\
\hline Phan Duc Tinh & & & 2 & 1964 \\
\hline Phan Duc Tuan & 1980 & $11 ; 30 ; 32$ & 6 & 2004 \\
\hline Phan H. Giang & 1963 & $68 ; 62 ; 91$ & 3 & 1994 \\
\hline Phan Hoang Chon & & $55 ; 57$ & 2 & 2011 \\
\hline Phan Hong Tin & 1981 & 16 & 2 & 2013 \\
\hline Phan Huu San & 1950 & & 2 & 1992 \\
\hline Phan Huy Khai & 1951 & & 20 & 1981 \\
\hline Phan Huy Phu & 1951 & & 2 & 1986 \\
\hline Phan Huy Thien & 1956 & 46 & 1 & 1994 \\
\hline Phan Le $\mathrm{Na}$ & & $60 ; 34$ & 3 & 1997 \\
\hline Phan M Hung & & $49 ; 90 ; 65$ & 23 & 2011 \\
\hline
\end{tabular}




\begin{tabular}{|c|c|c|c|c|}
\hline Phan Minh Dung & 1954 & & 8 & 1986 \\
\hline Phan Ngoc Vinh & & $76 ; 35$ & 1 & 2002 \\
\hline Phan Nhat Tinh & 1962 & $49 ; 90 ; 47$ & 11 & 1998 \\
\hline Phan Phien & & $53 ; 14$ & 1 & 2014 \\
\hline Phan Quoc Hung & 1984 & $35 ; 58 ; 53$ & 9 & 2008 \\
\hline Phan Quoc Khanh & 1946 & $90 ; 49 ; 47$ & 127 & 1979 \\
\hline Phan Tang Da & & & 3 & 1972 \\
\hline Phan Thanh An & 1968 & $52 ; 26 ; 65$ & 38 & 1996 \\
\hline Phan Thanh Hong & & & 3 & 2018 \\
\hline Phan Thanh Nam & 1974 & $34 ; 30 ; 37$ & 15 & 2005 \\
\hline Phan Thanh Tung & & 41 & 2 & 2019 \\
\hline Phan Thanh Viet & & $46 ; 35$ & 1 & 2020 \\
\hline Phan Thi Ha Duong & 1973 & $05 ; 68 ; 06$ & 32 & 2000 \\
\hline Phan Thi Ha Trang & & & 1 & 2018 \\
\hline Phan Thi Huong & & $60 ; 65 ; 34$ & 4 & 2018 \\
\hline Phan Thi Khanh Van & & 35 & 3 & 2019 \\
\hline Phan Thi Thuy & & $05 ; 13$ & 2 & 2019 \\
\hline Phan Thien Danh & & & 3 & 1996 \\
\hline Phan Thien Nhan & 1952 & & 2 & 1997 \\
\hline Phan Thien Thach & & $90 ; 49 ; 55$ & 40 & 1985 \\
\hline Phan Thoai & & & 1 & 1986 \\
\hline Phan Tri Kien & & 60 & 2 & 2020 \\
\hline Phan Trong Tien & & $49 ; 35$ & 1 & 2020 \\
\hline Phan Trung Hieu & & $42 ; 35 ; 34$ & 1 & 2011 \\
\hline Phan Trung Huy & & $68 ; 20 ; 94$ & 12 & 1992 \\
\hline Phan Tu Vuong & 1983 & $47 ; 49 ; 65$ & 6 & 2012 \\
\hline Phan Van Chuong & 1934 & $45 ; 54 ; 47$ & 26 & 1966 \\
\hline Phan Van Hap & 1938 & $65 ; 46 ; 47$ & 13 & 1965 \\
\hline Phan Van Thien & 1964 & 13 & 3 & 1999 \\
\hline Phan Van Tri & & & 4 & 2014 \\
\hline
\end{tabular}




\begin{tabular}{|c|c|c|c|c|}
\hline Phan Van Tuoc & 1978 & $35 ; 47 ; 55$ & 5 & 2004 \\
\hline Phan Viet Thu & 1951 & $46 ; 81$ & 4 & 1993 \\
\hline Phan Xuan Thanh & 1981 & $65 ; 35$ & 7 & 2012 \\
\hline Phi Ha & & $34 ; 15 ; 39$ & 2 & 2018 \\
\hline Pho Duc Tai & 1979 & $14 ; 32$ & 5 & 2001 \\
\hline Pho Duc Tru & & & 1 & 1974 \\
\hline Pho Huy Tai & 1972 & $14 ; 32$ & 1 & 2000 \\
\hline Phong Thi Thu Huyen & 1989 & $51 ; 65 ; 52$ & 1 & 2019 \\
\hline Phung Ho Hai & 1970 & $16 ; 14 ; 17$ & 28 & 1999 \\
\hline Phung Minh Duc & & $65 ; 90$ & 2 & 2016 \\
\hline Phung Van Manh & & $41 ; 32 ; 26$ & 9 & 2006 \\
\hline Pierre N.V. Tu & & & 1 & 1994 \\
\hline Quach Van Chuong & & & 1 & 2020 \\
\hline Quang Ha (Q.P. Ha or Q. Ha) & & & 3 & 2009 \\
\hline Sa Thi Lan Anh & 1988 & & 1 & 2018 \\
\hline Si Duc Quang & 1981 & $32 ; 30 ; 14$ & 52 & 2004 \\
\hline T. T. Khanh & & 35 & 1 & 2010 \\
\hline Ta Duy Phuong & 1952 & $90 ; 49 ; 65$ & 12 & 1985 \\
\hline Ta Hong Quang & 1957 & & 2 & 1986 \\
\hline Ta Khac $\mathrm{Cu}$ & & $54 ; 60 ; 58$ & 2 & 1989 \\
\hline Ta Le Loi & 1960 & $14 ; 32 ; 03$ & 13 & 1994 \\
\hline Ta Ngoc Anh & & $47 ; 60 ; 54$ & 2 & 2010 \\
\hline Ta Ngoc Cau & & & 1 & 1989 \\
\hline Ta Ngoc Tri & & $42 ; 65 ; 44$ & 3 & 2002 \\
\hline Ta Quang Son & & $90 ; 65 ; 41$ & 4 & 2007 \\
\hline Ta Quoc Bao & & $60 ; 45$ & 2 & 2003 \\
\hline Ta Thi Hoai An & 1971 & $30 ; 11 ; 12$ & 30 & 2001 \\
\hline Ta Thi Hong Yen & & 35 & 1 & 2011 \\
\hline Ta Thi Huyen Trang & & $34 ; 93 ; 49$ & 2 & 2016 \\
\hline Ta Thi Thanh Mai & & 41 & 1 & 2020 \\
\hline
\end{tabular}




\begin{tabular}{|c|c|c|c|c|}
\hline Ta Van Dinh & 1949 & $65 ; 35$ & 14 & 1964 \\
\hline Ta Van Tu & & 90 & 4 & 2000 \\
\hline Ta Viet Ton & & $47 ; 60 ; 92$ & 1 & 2010 \\
\hline Tang Quoc Bao & & $35 ; 37 ; 92$ & 34 & 2010 \\
\hline Tang Thi Ha Yen & & 90 & 1 & 2007 \\
\hline Tang Van Long & 1976 & $32 ; 31 ; 46$ & 7 & 2002 \\
\hline Thai Doan Chuong & 1979 & $49 ; 90 ; 65$ & 6 & 2010 \\
\hline Thai Quynh Phong & 1953 & $90 ; 49$ & 6 & 1990 \\
\hline Thai Thi Kim Chung & 1983 & 35 & 2 & 2019 \\
\hline Thai Thuan Quang & 1966 & $46 ; 32 ; 30$ & 15 & 1995 \\
\hline Thai Trung Hieu & & $20 ; 39$ & 1 & 2011 \\
\hline Than Quang Khoat & & 68 & 1 & 2011 \\
\hline Than Van Dinh & & $49 ; 47$ & 1 & 2020 \\
\hline Thanh V. V & & & 3 & 1993 \\
\hline Thieu Dinh Phong & 1980 & 13 & 1 & 2015 \\
\hline $\begin{array}{c}\text { Thieu Thi Kim Thoa (Thoa } \\
\text { Thieu) }\end{array}$ & & $65 ; 35$ & 1 & 2020 \\
\hline Thuc Manh Le & & $37 ; 39 ; 92$ & 2 & 2011 \\
\hline Thuy Do & 1995 & & 2 & 2017 \\
\hline Tit Bau & & 70 & 1 & 1997 \\
\hline To Tat Dat & 1976 & $35 ; 32$ & 2 & 2019 \\
\hline Ton Quoc Binh & 1954 & & 1 & 1987 \\
\hline Ton That Tri & 1956 & 20 & 8 & 1995 \\
\hline Tong Thanh Trung & 1974 & $92 ; 93 ; 65$ & 3 & 2007 \\
\hline Tong Viet Phi Hung & 1978 & $20 ; 05$ & 55 & 2003 \\
\hline Tra Quoc Khanh & & $47 ; 65 ; 31$ & 3 & 2020 \\
\hline Tran An Hai & & $30 ; 32$ & 4 & 2018 \\
\hline Tran An Hai & & & 1 & 2008 \\
\hline Tran Bao Anh & & $34 ; 47$ & 1 & 2008 \\
\hline Tran Bao Ngoc & & $35 ; 26 ; 47$ & 16 & 2018 \\
\hline Tran Cao Nguyen & & & 1 & 1986 \\
\hline
\end{tabular}




\begin{tabular}{|c|c|c|c|c|}
\hline Tran Dan Thu & 1966 & 05 & 4 & 1993 \\
\hline Tran Dang Hong & & & 1 & 1986 \\
\hline Tran Dang Hung & & & 1 & 2013 \\
\hline Tran Dang Phuc & 1997 & & 1 & 2016 \\
\hline Tran Dao Dong & 1955 & $53 ; 22 ; 14$ & 6 & 1989 \\
\hline Tran Dinh Duc & & $11 ; 30$ & 2 & 2008 \\
\hline Tran Dinh Ke & 1972 & $35 ; 47 ; 34$ & 14 & 2004 \\
\hline Tran Dinh Khang & & $68 ; 91$ & 2 & 1999 \\
\hline Tran Dinh Long & 1963 & 47 & 1 & 2002 \\
\hline Tran Dinh Luong & & $16 ; 55$ & 1 & 2007 \\
\hline Tran Dinh Phung & & 26 & 1 & 2017 \\
\hline Tran Dinh Quoc & & $90 ; 65 ; 49$ & 8 & 2004 \\
\hline Tran Dinh Thanh & & $45 ; 49 ; 47$ & 3 & 2002 \\
\hline Tran Dinh Tuong & & $60 ; 37 ; 92$ & 3 & 2017 \\
\hline Tran Dong Xuan & & $35 ; 46 ; 65$ & 2 & 2018 \\
\hline Tran Duc Anh & 1987 & $32 ; 30$ & 2 & 2016 \\
\hline Tran Duc Dung & 1988 & 13 & 1 & 2019 \\
\hline Tran Duc Khanh & & & 1 & 2013 \\
\hline Tran Duc Van & 1951 & $35 ; 46 ; 34$ & 60 & 1975 \\
\hline Tran Gia Lich & 1946 & $35 ; 76$ & 2 & 1992 \\
\hline Tran Gia Loc & 1959 & $39 ; 33$ & 1 & 2012 \\
\hline Tran Giang Nam & 1982 & $16 ; 18 ; 06$ & 19 & 2007 \\
\hline Tran Hoang Yen & & 65 & 1 & 1990 \\
\hline Tran Hong Mo & 1979 & $39 ; 46 ; 49$ & 10 & 2012 \\
\hline Tran Hong Thai & & 39 & 1 & 2014 \\
\hline Tran Hue Minh & 1977 & 32 & 4 & 2004 \\
\hline Tran Hue Nuong & 1959 & $49 ; 90$ & 8 & 1974 \\
\hline Tran Hung Cuong & & & 2 & 2019 \\
\hline Tran Hung Thao & 1940 & $60 ; 93$ & 15 & 1982 \\
\hline Tran Huu Nam & & & 4 & 1996 \\
\hline
\end{tabular}




\begin{tabular}{|c|c|c|c|c|}
\hline Tran Huy Ho & 1942 & 35 & 2 & 1985 \\
\hline Tran Huy Hoang & & & 1 & 1978 \\
\hline Tran Kim Chi & & & 4 & 1980 \\
\hline Tran Kim Dung & & & 1 & 1995 \\
\hline Tran Kim Thanh & 1973 & $60 ; 62$ & 3 & 1996 \\
\hline Tran Kim Tu (Chan Kim T'i) & & & 2 & 1987 \\
\hline Tran Le Nam & 1983 & 53 & 1 & 2008 \\
\hline Tran Loc Hung & 1954 & $60 ; 62 ; 68$ & 9 & 2001 \\
\hline Tran Loc Hung & & $60 ; 41$ & 3 & 2019 \\
\hline Tran Luu Cuong & & & 1 & 1993 \\
\hline Tran Manh Cuong & & $60 ; 37$ & 2 & 2009 \\
\hline Tran Manh Tuan & & 13 & 1 & 2011 \\
\hline Tran Minh Binh & & $35 ; 47 ; 82$ & 5 & 2009 \\
\hline Tran Minh Hoang & & $30 ; 35$ & 1 & 2008 \\
\hline Tran Minh Ngoc & & 60 & 3 & 2005 \\
\hline Tran Minh Nguyet & & $49 ; 35 ; 76$ & 4 & 2016 \\
\hline Tran Minh Phuong & 1982 & $35 ; 65 ; 78$ & 12 & 2017 \\
\hline Tran Minh Thuyet & & $46 ; 35 ; 34$ & 4 & 1999 \\
\hline Tran Minh Tuoc & 1970 & 05 & 2 & 2003 \\
\hline Tran Nam Trung & 1979 & $13 ; 05 ; 14$ & 21 & 2008 \\
\hline Tran Ngoc & & $47 ; 35$ & 1 & 2020 \\
\hline Tran Ngoc Danh & 1971 & $06 ; 05$ & 4 & 1996 \\
\hline Tran Ngoc Diem & & 35 & 5 & 1997 \\
\hline Tran Ngoc Giao & 1949 & 32 & 5 & 1991 \\
\hline Tran Ngoc Ha & & & 1 & 2014 \\
\hline Tran Ngoc Hoi & & & 5 & 1987 \\
\hline Tran Ngoc Lien & & $65 ; 30 ; 33$ & 4 & 2003 \\
\hline Tran Ngoc Minh & & & 1 & 1993 \\
\hline Tran Ngoc Nam & 1970 & 55 & 4 & 2000 \\
\hline Tran Ngoc Nhat Huyen & 1996 & & 1 & 2020 \\
\hline
\end{tabular}




\begin{tabular}{|c|c|c|c|c|}
\hline Tran Ngoc Tam & & $91 ; 90 ; 49$ & 6 & 2012 \\
\hline Tran Ngoc Thach & & $35 ; 47 ; 62$ & 14 & 2018 \\
\hline Tran Ngoc Tuan & & $34 ; 93$ & 1 & 2019 \\
\hline Tran Nguyen An & & 13 & 1 & 2019 \\
\hline Tran Nguyen Binh & 1984 & & 1 & 2020 \\
\hline Tran Nhat Luan & & $31 ; 47 ; 65$ & 3 & 2020 \\
\hline Tran Ninh Hoa & & $90 ; 49$ & 4 & 2005 \\
\hline Tran Phuong Thuy Lam & & $45 ; 35$ & 1 & 2020 \\
\hline Tran Quan Ky & & $60 ; 34 ; 92$ & 1 & 2020 \\
\hline Tran Quang Hung & & 32 & 1 & 2019 \\
\hline Tran Quang Vinh & 1973 & 60 & 3 & 2001 \\
\hline Tran Quoc Binh & 1969 & $47 ; 65 ; 54$ & 8 & 1996 \\
\hline Tran Quoc Chien & 1950 & & 3 & 1989 \\
\hline Tran Quoc Cong & & $52 ; 32$ & 1 & 2014 \\
\hline Tran Quoc Duy & & $49 ; 90$ & 1 & 2016 \\
\hline Tran Quoc Tuan & & $35 ; 34$ & 1 & 2020 \\
\hline Tran Quoc Viet & & & 1 & 1984 \\
\hline Tran Quoc Viet (DTU) & & $35 ; 62 ; 65$ & 10 & 2013 \\
\hline Tran Quyet Thang & 1955 & & 5 & 1987 \\
\hline Tran Tat Dat & 1982 & $34 ; 26$ & 5 & 2005 \\
\hline Tran Thai An Nghia & 1979 & $49 ; 90 ; 65$ & 23 & 2008 \\
\hline Tran Thai Bao & & 93 & 1 & 2007 \\
\hline Tran Thai Duong & & 05 & 1 & 2015 \\
\hline Tran Thai Son & & & 2 & 1986 \\
\hline Tran Thanh & & & 1 & 1990 \\
\hline Tran Thanh (UNSW) & & $35 ; 47 ; 65$ & 4 & 2015 \\
\hline Tran Thanh Binh & & $47 ; 35$ & 2 & 2020 \\
\hline Tran Thanh Binh (SGU) & & $35 ; 47 ; 92$ & 12 & 2014 \\
\hline Tran Thanh Tuan & 1980 & & 1 & 2017 \\
\hline Tran Thanh Tung & & $34 ; 93$ & 5 & 2006 \\
\hline
\end{tabular}




\begin{tabular}{|c|c|c|c|c|}
\hline Tran The Anh & & $45 ; 34$ & 1 & 2018 \\
\hline Tran The Hung & & $35 ; 47 ; 42$ & 4 & 2015 \\
\hline Tran The Hung & & & 1 & 2015 \\
\hline Tran Thi Hue & 1958 & $49 ; 90 ; 65$ & 4 & 1993 \\
\hline Tran Thi Huong & & $47 ; 49$ & 1 & 2020 \\
\hline Tran Thi Huong Anh & & $65 ; 90$ & 1 & 2017 \\
\hline Tran Thi Huyen Thanh & & $90 ; 65 ; 49$ & 2 & 2020 \\
\hline Tran Thi Khieu & & $31 ; 65 ; 47$ & 2 & 2020 \\
\hline Tran Thi Kim Chi & 1945 & 34 & 5 & 1991 \\
\hline Tran Thi Lan Anh & & $47 ; 54$ & 4 & 1999 \\
\hline Tran Thi Le & 1949 & 35 & 8 & 1987 \\
\hline Tran Thi Loan & & $34 ; 92 ; 35$ & 9 & 1999 \\
\hline Tran Thi Loan & & 35 & 1 & 2010 \\
\hline Tran Thi Mai & & $49 ; 90 ; 91$ & 3 & 2018 \\
\hline Tran Thi Phuong & 1982 & 13 & 2 & 2004 \\
\hline Tran Thi Thu Huong & 1984 & $91 ; 05 ; 68$ & 4 & 2010 \\
\hline Tran Thi Tu Trinh & & 49 & 1 & 2020 \\
\hline Tran Thien Thanh & 1983 & $62 ; 60$ & 1 & 2008 \\
\hline Tran Thu Ha & & & 1 & 1992 \\
\hline Tran Thu Le & & & 1 & 2020 \\
\hline Tran Tin Kiet & 1950 & $93 ; 34$ & 3 & 2000 \\
\hline Tran Tri Dung & & $47 ; 42 ; 35$ & 2 & 2020 \\
\hline Tran Tri Kiet & 1965 & $46 ; 30$ & 3 & 2004 \\
\hline Tran Trinh Minh Son & & $90 ; 49 ; 91$ & 2 & 2013 \\
\hline Tran Trong Nguyen & 1972 & $60 ; 92$ & 3 & 2001 \\
\hline Tran Trung & & & 1 & 2020 \\
\hline Tran Tuan Nam & 1965 & $13 ; 16$ & 8 & 2000 \\
\hline Tran Tuyen & & $49 ; 90$ & 4 & 2017 \\
\hline Tran Van An & 1965 & $54 ; 46$ & 13 & 1993 \\
\hline Tran Van Bang & 1974 & $35 ; 49$ & 3 & 2006 \\
\hline
\end{tabular}




\begin{tabular}{|c|c|c|c|c|}
\hline Tran Van Dung & 1955 & 20 & 4 & 1993 \\
\hline Tran Van Hoai & 1972 & & 7 & 2006 \\
\hline $\begin{array}{c}\text { Tran Van Hung (Chan Van } \\
\text { Khung) }\end{array}$ & & & 1 & 1994 \\
\hline \multicolumn{2}{|c|}{ Tran Van Huyen (Tr\cfgrv an Huyen) } & & 1 & 1982 \\
\hline Tran Van Lang & 1959 & & 1 & 1996 \\
\hline Tran Van Nam & & $03 ; 15 ; 65$ & 3 & 2019 \\
\hline Tran Van Nghi & & 90 & 2 & 2017 \\
\hline Tran Van Nhung & 1948 & $34 ; 60 ; 93$ & 18 & 1979 \\
\hline Tran Van Su & & $90 ; 49 ; 45$ & 3 & 2020 \\
\hline Tran Van Tan & 1976 & $30 ; 32 ; 11$ & 14 & 2005 \\
\hline Tran Van Thang & & $90 ; 49 ; 47$ & 4 & 2011 \\
\hline Tran Van Thuy & & 32 & 2 & 2018 \\
\hline Tran Van Truong & & 47 & 1 & 2000 \\
\hline Tran Viet Anh & & $90 ; 65 ; 49$ & 6 & 2016 \\
\hline Tran Viet Dung & 1961 & & 3 & 1990 \\
\hline Tran Vinh Hien & & & 1 & 1974 \\
\hline Tran Vinh Hung & 1984 & $58 ; 47 ; 35$ & 3 & 2007 \\
\hline Tran Vinh Linh & & & 3 & 2009 \\
\hline Tran Vu Khanh & 1983 & $32 ; 47 ; 37$ & 8 & 2007 \\
\hline Tran Vu Thieu & 1941 & $90 ; 65 ; 49$ & 17 & 1980 \\
\hline Tran Vui & 1960 & $14 ; 22 ; 53$ & 6 & 1989 \\
\hline Tran Xuan Khoi & 1976 & & 1 & 2017 \\
\hline Tran Xuan Loc & & $35 ; 78$ & 2 & 2019 \\
\hline Tran Xuan Sinh & 1945 & & 1 & 1997 \\
\hline Tran Xuan Tiep & 1957 & & 1 & 1990 \\
\hline Trieu Quynh Trang & 1984 & & 1 & 2018 \\
\hline Trieu Van Dung & & 32 & 1 & 2020 \\
\hline Trinh Anh Ngoc & 1963 & & 1 & 1995 \\
\hline Trinh Cong Dieu & 1956 & $93 ; 47 ; 46$ & 4 & 1989 \\
\hline Trinh Dao Chien & 1962 & 30 & 1 & 2000 \\
\hline
\end{tabular}




\begin{tabular}{|c|c|c|c|c|}
\hline Trinh Duc Tai & 1966 & $39 ; 58 ; 26$ & 2 & 2012 \\
\hline Trinh Khanh Duy & 1985 & $65 ; 37$ & 1 & 2007 \\
\hline Trinh Ngoc Hai & & $65 ; 90 ; 47$ & 3 & 2017 \\
\hline Trinh Ngoc Minh & & & 3 & 1985 \\
\hline Trinh Thanh Deo & & 16 & 4 & 2012 \\
\hline Trinh Thi Minh Hang & & 35 & 3 & 2009 \\
\hline Trinh Tuan & & $45 ; 44 ; 33$ & 5 & 2010 \\
\hline Trinh Tuan & & $44 ; 33$ & 3 & 2003 \\
\hline Trinh Tuan Anh & 1971 & $92 ; 34 ; 39$ & 14 & 1997 \\
\hline Trinh Tuan Phong & 1986 & 35 & 2 & 2008 \\
\hline Trinh Tung & & & 1 & 2015 \\
\hline Trinh Viet Duoc & 1985 & $35 ; 34 ; 37$ & 7 & 2014 \\
\hline Trung Truong & 1989 & $35 ; 65 ; 78$ & 1 & 2020 \\
\hline Truong Chi Tin & & & 1 & 1989 \\
\hline Truong Chi Trung & & $22 ; 20 ; 46$ & 2 & 1997 \\
\hline Truong Cong Quynh & 1981 & 16 & 6 & 2007 \\
\hline Truong Dinh Tu & & 16 & 1 & 2020 \\
\hline Truong Ha Hai & 1967 & $35 ; 65$ & 1 & 2011 \\
\hline Truong Hong Minh & & $37 ; 32 ; 53$ & 1 & 2013 \\
\hline Truong Minh Tuyen & & $47 ; 49 ; 90$ & 4 & 2019 \\
\hline Truong My Dung & & & 2 & 1987 \\
\hline Truong Ngoc Son & & & 1 & 2002 \\
\hline Truong Quang Bao & & $90 ; 49 ; 46$ & 31 & 2003 \\
\hline Truong Thi Hieu Hanh & & & 1 & 2020 \\
\hline Truong Thi Hong Thanh & & $14 ; 13$ & 1 & 2020 \\
\hline Truong Thi Thanh Phuong & & 90 & 1 & 2020 \\
\hline Truong Thi Thuy Duong & 1982 & $90 ; 49 ; 91$ & 4 & 2010 \\
\hline Truong Trong Nghia & & $47 ; 35$ & 1 & 2015 \\
\hline Truong Trung Tuyen & 1980 & $30 ; 74 ; 47$ & 6 & 2003 \\
\hline Truong Van Thuong & 1955 & 46 & 4 & 1999 \\
\hline
\end{tabular}




\begin{tabular}{|c|c|c|c|c|}
\hline Truong Viet Dung & 1952 & & 1 & 2009 \\
\hline Truong Vinh An & & $34 ; 47$ & 4 & 2013 \\
\hline Truong Xuan Duc Ha & 1957 & $49 ; 90 ; 34$ & 38 & 1980 \\
\hline Tu Nguyen & & & 1 & 2019 \\
\hline Ung Ngoc Quang & & & 2 & 1994 \\
\hline Van Duc Trung & & $13 ; 14$ & 1 & 2019 \\
\hline Van Duong Dinh & & 35 & 3 & 2020 \\
\hline Vo Anh Khoa & & $35 ; 65 ; 47$ & 27 & 2015 \\
\hline Vo Bay & & & 1 & 2013 \\
\hline Vo Dang Thao & 1954 & 30 & 8 & 1976 \\
\hline Vo Duc Cam Hai & & $35 ; 65$ & 1 & 2019 \\
\hline Vo Giang Giai & 1972 & 35 & 4 & 2007 \\
\hline Vo Hanh Nguyen & & 42 & 1 & 2020 \\
\hline Vo Hoang Hung & & $35 ; 92$ & 1 & 2020 \\
\hline Vo Minh Pho & 1946 & $90 ; 52 ; 47$ & 3 & 2010 \\
\hline Vo Minh Tam & & $47 ; 49 ; 74$ & 2 & 2020 \\
\hline Vo Ngoc Thieu & & & 1 & 2020 \\
\hline Vo Si Trong Long & 1981 & $47 ; 49 ; 90$ & 6 & 2011 \\
\hline Vo Thanh Tung & 1979 & & 2 & 2000 \\
\hline Vo Thi Bich Khue & 1982 & $46 ; 47 ; 15$ & 2 & 2018 \\
\hline Vo Thi Le Hang & 1986 & $54 ; 47 ; 46$ & 2 & 2015 \\
\hline Vo Thi Ngoc Chau & & & 2 & 2016 \\
\hline Vo Thi Nhat Minh & & $35 ; 93$ & 1 & 2016 \\
\hline Vo Thi Nhu Quynh & & 55 & 1 & 2007 \\
\hline Vo Thi Thu Hien & 1977 & $35 ; 47$ & 2 & 2008 \\
\hline Vo Thi Truc Giang & & $62 ; 60$ & 1 & 2020 \\
\hline Vo Van $\mathrm{Au}$ & & $35 ; 47 ; 65$ & 26 & 2017 \\
\hline Vo Van Tai & & $62 ; 68$ & 1 & 2020 \\
\hline Vo Van Tan & & & 1 & 2002 \\
\hline Vo Viet Can & & & 1 & 1980 \\
\hline
\end{tabular}




\begin{tabular}{|c|c|c|c|c|}
\hline Vo Viet Tri & & $47 ; 35 ; 34$ & 4 & 2018 \\
\hline $\mathrm{Vu} \mathrm{A.} \mathrm{Ha}$ & & & 1 & 1998 \\
\hline Vu Anh My & 1984 & $65 ; 60$ & 2 & 2020 \\
\hline Vu Chi Cuong & & & 1 & 2012 \\
\hline Vu Cong Bang & 1983 & $90 ; 42 ; 47$ & 2 & 2006 \\
\hline Vu Dinh Hoa & 1956 & 05 & 11 & 1983 \\
\hline Vu Duc Nghia & & 06 & 1 & 2004 \\
\hline Vu Duc Thi & 1949 & $68 ; 05$ & 32 & 1985 \\
\hline Vu Duc Thinh & & $49 ; 90 ; 65$ & 1 & 2020 \\
\hline Vu Duc Viet & 1988 & & 2 & 2014 \\
\hline Vu Duy Man & 1950 & & 1 & 1978 \\
\hline Vu Duy Manh & & & 1 & 2019 \\
\hline Vu Ha Van & 1970 & $05 ; 60 ; 11$ & 144 & 1996 \\
\hline Vu Hai Sam & 1979 & $60 ; 34 ; 92$ & 2 & 2006 \\
\hline $\mathrm{Vu} \mathrm{Ho}$ & & 34 & 7 & 2012 \\
\hline Vu Hoai An & 1960 & $30 ; 11 ; 32$ & 12 & 2002 \\
\hline Vu Hoang Linh & 1968 & $34 ; 93 ; 65$ & 13 & 1996 \\
\hline Vu Huu Nhu & 1983 & $49 ; 35 ; 65$ & 5 & 2010 \\
\hline Vu Huy Tinh & & & 1 & 1983 \\
\hline Vu Kim Tuan & 1960 & $44 ; 65 ; 45$ & 74 & 1981 \\
\hline Vu Manh Toi & & $35 ; 93 ; 76$ & 14 & 2011 \\
\hline Vu Ngoan & & & 1 & 1965 \\
\hline Vu Ngoc Phat & 1953 & $93 ; 34 ; 49$ & 144 & 1980 \\
\hline Vu Nguyen Son Tung & & & 1 & 2020 \\
\hline Vu Nhat Huy & & $46 ; 26 ; 41$ & 19 & 2009 \\
\hline Vu Nhu Lan & 1951 & $90 ; 93$ & 1 & 2008 \\
\hline Vu Quang Bach & & $76 ; 35 ; 82$ & 1 & 2020 \\
\hline Vu Quang Hung & & $46 ; 47$ & 1 & 2005 \\
\hline Vu Quang Thanh (Thanh Vu) & 1986 & 13 & 6 & 2015 \\
\hline Vu Quoc Phong & 1954 & $47 ; 34 ; 35$ & 47 & 1977 \\
\hline
\end{tabular}




\begin{tabular}{|c|c|c|c|c|}
\hline Vu Quoc Thanh & & $34 ; 41 ; 28$ & 4 & 1990 \\
\hline Vu The Khoi & 1972 & $57 ; 20 ; 33$ & 14 & 1996 \\
\hline Vu Thi Hien Anh & & 35 & 1 & 2020 \\
\hline Vu Thi Hong Thanh & & $28 ; 26 ; 30$ & 5 & 1999 \\
\hline Vu Thi Huong & 1990 & $49 ; 46 ; 91$ & 3 & 2017 \\
\hline Vu Thi Mai & & $76 ; 35$ & 2 & 2017 \\
\hline Vu Thi Ngoc Anh & 1990 & & 1 & 2018 \\
\hline Vu Thi Ngoc Anh & & 60 & 1 & 2020 \\
\hline Vu Thi Ngoc Ha & & $35 ; 30 ; 34$ & 7 & 2005 \\
\hline Vu Thi Thu Ha & & $53 ; 57$ & 1 & 2001 \\
\hline Vu Thi Thu Huong & & & 1 & 2005 \\
\hline Vu Thi Thuy Duong & & $35 ; 76$ & 1 & 2020 \\
\hline Vu Thien Ban & & & 3 & 1983 \\
\hline Vu Tien Dung & 1981 & $47 ; 65 ; 68$ & 4 & 2018 \\
\hline Vu Tien Viet & 1955 & $37 ; 65$ & 1 & 2007 \\
\hline Vu Trong Luong & & $35 ; 34 ; 47$ & 4 & 2016 \\
\hline Vu Trong Tuan & 1935 & 58 & 2 & 1973 \\
\hline Vu Tuan & 1935 & $34 ; 35$ & 15 & 1963 \\
\hline Vu Van Dat & 1950 & & 1 & 1982 \\
\hline Vu Van Dong & & 90 & 2 & 2018 \\
\hline Vu Van Khu & 1988 & $60 ; 92$ & 1 & 2012 \\
\hline Vu Van Khuong & & 39 & 1 & 2014 \\
\hline Vu Van Truong & 1975 & & 1 & 2017 \\
\hline Vu Viet Hung & 1983 & $32 ; 14$ & 5 & 2012 \\
\hline Vu Viet Yen & & $60 ; 54$ & 7 & 1988 \\
\hline Vu Vinh Quang & & $30 ; 35 ; 65$ & 2 & 2004 \\
\hline Vuong Mai Phuong & & & 3 & 2018 \\
\hline Vuong Manh Son & & $17 ; 22$ & 3 & 1979 \\
\hline Vuong Nguyen Doan & & $65 ; 46 ; 47$ & 2 & 2017 \\
\hline Vuong Quan Hoang & 1972 & 60 & 3 & 2005 \\
\hline
\end{tabular}




(20)

1

1

Bảng B. Số lượng tác phẩm công bố trên các tạp chí uy tín theo năm

\begin{tabular}{|c|c|c|c|c|}
\hline Tạp chí & Năm & $\begin{array}{c}\text { Số } \\
\text { bài }\end{array}$ & Tác giả & Ngành \\
\hline $\begin{array}{c}\text { Annales scientifiques de l'École Normale } \\
\text { Supérieure }\end{array}$ & 1950 & 1 & Le Van Thiem & 30 \\
\hline Annals of Mathematics & 1976 & 1 & Nguyen Huu Anh & \\
\hline Duke Mathematical Journal & 1977 & 1 & Duong Hong Phong & $32,35,46$ \\
\hline Duke Mathematical Journal & 1983 & 1 & Ha Huy Khoai & \\
\hline Inventiones mathematicae & 1986 & 1 & Duong Hong Phong & 42 \\
\hline Acta Mathematica & 1986 & 1 & Duong Hong Phong & $42,44,47,58$ \\
\hline Duke Mathematical Journal & 1989 & 1 & Duong Hong Phong & 42,44 \\
\hline Advances in Mathematics & 1992 & 1 & Ngo Viet Trung & \\
\hline Inventiones mathematicae & 1992 & 1 & Duong Hong Phong & $35,42,47$ \\
\hline Annals of Mathematics & 1994 & 1 & Duong Hong Phong & 35,43 \\
\hline Journal für die reine und angewandte Mathematik & 1997 & 1 & Ngo Viet Trung & \\
\hline Acta Mathematica & 1997 & 1 & Duong Hong Phong & 35,47 \\
\hline Advances in Mathematics & 1998 & 1 & Duong Hong Phong & $35,42,47$ \\
\hline $\begin{array}{c}\text { Annales scientifiques de l'École Normale } \\
\text { Supérieure }\end{array}$ & 1999 & 1 & Ngo Bao Chau & \\
\hline Duke Mathematical Journal & 1999 & 1 & Ngo Bao Chau & \\
\hline Annals of Mathematics & 2000 & 1 & Duong Hong Phong & 11,32 \\
\hline Duke Mathematical Journal & 2000 & 1 & Vu Ha Van & 05,11 \\
\hline Advances in Mathematics & 2004 & 1 & Vu Ha Van & $05,60,74$ \\
\hline Annals of Mathematics & 2005 & 1 & Dinh Tien Cuong & \\
\hline Duke Mathematical Journal & 2005 & 1 & Vu Ha Van & 11 \\
\hline $\begin{array}{c}\text { Annales scientifiques de l'École Normale } \\
\text { Supérieure }\end{array}$ & 2006 & 1 & Ngo Bao Chau & \\
\hline
\end{tabular}




\begin{tabular}{|c|c|c|c|c|}
\hline Advances in Mathematics & 2006 & 1 & Vu Ha Van & \\
\hline Annals of Mathematics & 2006 & 1 & Vu Ha Van & \\
\hline Duke Mathematical Journal & 2006 & 1 & Vu Ha Van & 05 \\
\hline Inventiones mathematicae & 2006 & 2 & $\begin{array}{c}\text { Duong Hong Phong,Ngo } \\
\text { Bao Chau }\end{array}$ & 32,53 \\
\hline Journal of the American Mathematical Society & 2006 & 1 & Vu Ha Van & \\
\hline Advances in Mathematics & 2007 & 1 & Ngo Viet Trung & 13 \\
\hline Journal of the American Mathematical Society & 2007 & 1 & Vu Ha Van & \\
\hline Advances in Mathematics & 2008 & 2 & Phung Ho Hai,Vu Ha Van & 11 \\
\hline Journal für die reine und angewandte Mathematik & 2008 & 1 & Pham Ngoc Anh & 16,46 \\
\hline Annals of Mathematics & 2008 & 1 & Ngo Bao Chau & 14,22 \\
\hline Inventiones mathematicae & 2008 & 2 & $\begin{array}{c}\text { Duong Hong Phong,Pham } \\
\text { Huu Tiep }\end{array}$ & $20,32,53$ \\
\hline Advances in Mathematics & 2009 & 3 & $\begin{array}{c}\text { Pham Hoang Hiep,Pham } \\
\text { Huu Tiep,Vu Ha Van }\end{array}$ & $05,15,20$ \\
\hline Annals of Mathematics & 2009 & 1 & Vu Ha Van & 11 \\
\hline Duke Mathematical Journal & 2009 & 1 & $\begin{array}{c}\text { Nguyen Trong Toan } \\
\text { (Nguyen T. Toan) }\end{array}$ & 35 \\
\hline Acta Mathematica & 2009 & 1 & Dinh Tien Cuong & \\
\hline Advances in Mathematics & 2010 & 3 & $\begin{array}{c}\text { Dao Hai Long (Hailong } \\
\text { Dao),Nguyen Sum,Pham } \\
\text { Huu Tiep }\end{array}$ & $13,16,20,55$ \\
\hline Advances in Mathematics & 2011 & 3 & $\begin{array}{c}\text { Ngo Viet Trung,Nguyen } \\
\text { Cong Minh,Nguyen Huu } \\
\text { Hoi (Hoi H. Nguyen),Vu } \\
\text { Ha Van }\end{array}$ & 11 \\
\hline Annals of Mathematics & 2011 & 3 & $\begin{array}{c}\text { Nguyen Hoai Minh,Pham } \\
\text { Huu Tiep }\end{array}$ & $20,46,58$ \\
\hline Duke Mathematical Journal & 2011 & 1 & Nguyen Hoai Minh & 26,28 \\
\hline Inventiones mathematicae & 2011 & 2 & $\begin{array}{c}\text { Nguyen Hoai Minh,Pham } \\
\text { Huu Tiep }\end{array}$ & 26,46 \\
\hline Acta Mathematica & 2011 & 1 & Vu Ha Van & \\
\hline
\end{tabular}




\begin{tabular}{|c|c|c|c|c|}
\hline Advances in Mathematics & 2012 & 4 & $\begin{array}{c}\text { Dinh Tien Cuong,Ngo } \\
\text { Viet Trung,Nguyen Tien } \\
\text { Dung,Vu Ha Van }\end{array}$ & 13,53 \\
\hline Journal für die reine und angewandte Mathematik & 2012 & 1 & Pham Huu Tiep & \\
\hline Duke Mathematical Journal & 2012 & 2 & $\begin{array}{l}\text { Nguyen Huu Hoi (Hoi H. } \\
\text { Nguyen),Pham Huu Tiep }\end{array}$ & 11,20 \\
\hline Annals of Mathematics & 2013 & 2 & $\begin{array}{c}\text { Ngo Bao Chau,Pham Huu } \\
\text { Tiep }\end{array}$ & \\
\hline Advances in Mathematics & 2014 & 1 & Pham Huu Tiep & 20 \\
\hline Acta Mathematica & 2014 & 1 & Pham Hoang Hiep & 14,32 \\
\hline Advances in Mathematics & 2015 & 4 & $\begin{array}{c}\text { Dinh Tien Cuong,Nguyen } \\
\text { Duy Tan,Nguyen } \\
\text { Sum,Pham Huu Tiep }\end{array}$ & $12,20,55$ \\
\hline Journal für die reine und angewandte Mathematik & 2015 & 1 & Pham Huu Tiep & 20 \\
\hline Advances in Mathematics & 2016 & 4 & $\begin{array}{c}\text { Nguyen Trong Toan } \\
\text { (Nguyen T. Toan),Nguyen } \\
\text { Van Hoang (1985),Pham } \\
\text { Huu Tiep,Vu Ha Van }\end{array}$ & $\begin{array}{c}05,20,26,46, \\
52,60\end{array}$ \\
\hline Duke Mathematical Journal & 2016 & 1 & $\begin{array}{c}\text { Nguyen Trong Toan } \\
\text { (Nguyen T. Toan) }\end{array}$ & 35 \\
\hline Advances in Mathematics & 2017 & 3 & $\begin{array}{l}\text { Lu Hoang Chinh,Nguyen } \\
\text { Duy Tan,Pham Hung Quy }\end{array}$ & 13,53 \\
\hline Journal für die reine und angewandte Mathematik & 2017 & 1 & Lu Hoang Chinh & 32 \\
\hline Inventiones mathematicae & 2017 & 1 & Duong Hong Phong & $35,53,58$ \\
\hline Geometry and Topology & 2017 & 1 & Lu Hoang Chinh & 32,53 \\
\hline Inventiones mathematicae & 2018 & 2 & $\begin{array}{c}\text { Dinh Tien Cuong,Pham } \\
\text { Huu Tiep }\end{array}$ & 20 \\
\hline Acta Mathematica & 2018 & 1 & Pham Huu Tiep & 20 \\
\hline Advances in Mathematics & 2019 & 4 & $\begin{array}{l}\text { Pham Hung Quy,Pham } \\
\text { Huu Tiep,Vu Ha Van }\end{array}$ & 20 \\
\hline Journal für die reine und angewandte Mathematik & 2019 & 1 & Duong Hong Phong & 35,58 \\
\hline Annals of Mathematics & 2019 & 1 & Pham Huu Tiep & 11,20 \\
\hline Duke Mathematical Journal & 2019 & 1 & Dinh Tien Cuong & \\
\hline Inventiones mathematicae & 2019 & 1 & $\begin{array}{c}\text { Ngo Viet Trung,Nguyen } \\
\text { Dang Hop }\end{array}$ & \\
\hline
\end{tabular}




\begin{tabular}{|c|c|c|c|c|}
\hline $\begin{array}{c}\text { Annales scientifiques de l'École Normale } \\
\text { Supérieure }\end{array}$ & 2020 & 1 & Lu Hoang Chinh & 32,58 \\
\hline Advances in Mathematics & 2020 & 3 & $\begin{array}{c}\text { Nguyen Hoai Minh,Pham } \\
\text { Huu Tiep }\end{array}$ & $\begin{array}{c}20,26,35,46, \\
78,82\end{array}$ \\
\hline Journal für die reine und angewandte Mathematik & 2020 & 1 & Lu Hoang Chinh & \\
\hline
\end{tabular}

Bảng C. Số lượng tác phẩm và tạp chí uy tín hàng đầu các tác giả công bố

\begin{tabular}{|c|c|c|}
\hline Tác giả & Số bài & Số tạp chí \\
\hline Pham Huu Tiep & 21 & 6 \\
\hline Vu Ha Van & 17 & 5 \\
\hline Duong Hong Phong & 13 & 6 \\
\hline Dinh Tien Cuong & 6 & 5 \\
\hline Ngo Bao Chau & 6 & 4 \\
\hline Ngo Viet Trung & 6 & 3 \\
\hline Lu Hoang Chinh & 5 & 4 \\
\hline Nguyen Hoai Minh & 4 & 4 \\
\hline Nguyen Trong Toan (Nguyen T. Toan) & 3 & 2 \\
\hline Nguyen Huu Hoi (Hoi H. Nguyen) & 2 & 2 \\
\hline Nguyen Sum & 2 & 1 \\
\hline Nguyen Duy Tan & 2 & 1 \\
\hline Pham Hoang Hiep & 2 & 2 \\
\hline Pham Hung Quy & 2 & 1 \\
\hline Pham Ngoc Anh & 1 & 1 \\
\hline
\end{tabular}


AISDL

\begin{tabular}{|c|c|c|}
\hline Phung Ho Hai & 1 & 1 \\
\hline Nguyen Huu Anh & 1 & 1 \\
\hline Nguyen Tien Dung & 1 & 1 \\
\hline Nguyen Van Hoang (1985) & 1 & 1 \\
\hline Nguyen Cong Minh & 1 & 1 \\
\hline Nguyen Dang Hop & 1 & 1 \\
\hline Dao Hai Long (Hailong Dao) & 1 & 1 \\
\hline Ha Huy Khoai & 1 & 1 \\
\hline Le Van Thiem & & 1 \\
\hline
\end{tabular}


$\cos \cos \cos \cos \cos 030808080800$

\title{
NHÓM DƯ ÁN SCIMATH
}

\author{
Ngô Bảo Châu \\ Vương Quân Hoàng \\ Lã Việt Phương \\ Lê Tuấn Hoa \\ Lê Minh Hà \\ Trịnh Thị Thúy Giang \\ Phạm Hùng Hiệp \\ Nguyễn Thanh Thanh Huyên \\ Nguyễn Thanh Dung \\ Nguyễn Thị Linh \\ Trân Trung \\ Nguyễn Minh Hoàng \\ Hồ Mạnh Toàn
}

Dự án SciMath được sự hỗ trợ từ

Quỹ Đổi mới Sáng tạo Phenikaa

Chương trình Trọng điểm Quốc gia Phát triển Toán học 2010-2020 